\title{
A 5GHz Passively Interpolated 5-bit Time-to-Digital Converter with 8ps Resolution in IBM 130nm CMOS
}

\author{
by
}

\author{
Kiril Kidisyuk, B.Eng.
}

\author{
A thesis submitted to the \\ Faculty of Graduate Studies and Research \\ in partial fulfillment of the requirements for the degree of \\ Master of Applied Science in Electrical Engineering \\ Ottawa-Carleton Institute for Electrical and Computer Engineering \\ Department of Electronics \\ Carleton University \\ Ottawa, Ontario \\ March, 2019 \\ (C) Copyright
}

Kiril Kidisyuk, 2019 


\section{Abstract}

This work demonstrates the development of a 5-bit time to digital converter (TDC) using the local passive interpolation (LPI) technique. The TDC architecture achieves a high resolution, while maintaining a low conversion latency, and a good linearity over process variation at multi-GHz rate of operation, which simplifies the calibration process. The time-to-digital converter was fabricated in a $0.13 \mu \mathrm{m}$ IBM CMOS process (CMRF8SF). At a sampling rate of $100 \mathrm{MHz}$ the maximum frequency of operation was measured to be $1.6 \mathrm{GHz}$. The uncalibrated resolution of $8.1 \mathrm{psec}$ and a dynamic range of 260 psec were measured. The TDC is compatible with loop counter architectures that can further extend its dynamic range. The raw integral (INL) and differential (DNL) non-linearity of 1.02LSB and 0.52LSB respectively were observed. A correlation with the simulated results confirmed that the proposed LPI-TDC can

operate at $5 \mathrm{GHz}$ with some adjustments to measurement setup, input matching, and the on-chip supply integrity. 


\section{Acknowledgments}

I am deeply grateful to all the people who in one way or another have supported me during my Masters of Applied Science thesis. This academic research has been an invaluable experience, which has embarked me on a path to continue exploring integrated ciruits in the future. In particular, I would like to acknowledge Professor John W.M. Rogers and Professor Calvin Plett at Carleton University for their technical assistance in the research and writing of this thesis. Their oversight and feedback has allowed me to grow immensly, both professionally and as a researcher. The valuable guidance has created a firm foundation for all my future endeavours. I would also like to extend a special thank you to Nagui Mikhail for ensuring the testing phase of my research went smoothly and that I had all the components and equipment required. To my family and friends, thank you for your encouragement and support during this process, without which, this undertaking would not have been possible. 


\section{Contents}

Abstract ii

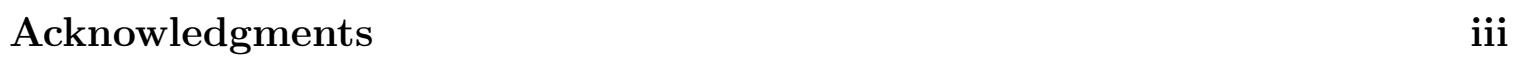

Table of Contents iv

List of Tables viii

List of Figures $\quad$ X

$\begin{array}{ll}\text { Nomenclature } & \text { XV }\end{array}$

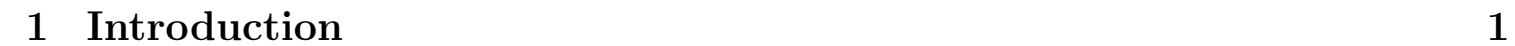

1.1 Motivation and Objectives . . . . . . . . . . . . . . . . . . 1

1.2 Contributions . . . . . . . . . . . . . . . . . 3

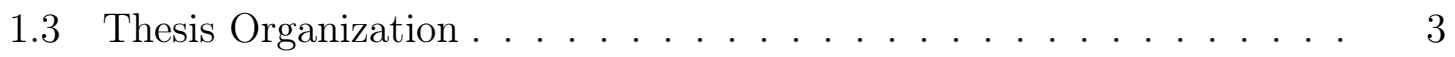

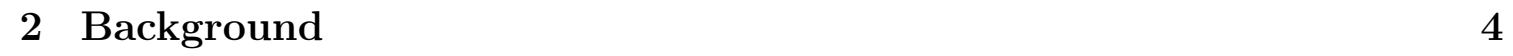

2.1 ADPLL Background $\ldots \ldots \ldots$. . . . . . . . . . . . . . 4

$2.1 .1 \quad$ Analog PLL Solution . . . . . . . . . . . . . . . . . . . 5

2.1 .2 All Digital PLL Solution . . . . . . . . . . . . . . . . . 6

2.2 First Generation TDC $\ldots \ldots \ldots \ldots \ldots$

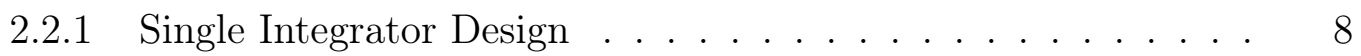


2.2 .2 Dual-slope Integrator Design . . . . . . . . . . . . . . . . . . . 8

2.3 Second Generation Digital TDC . . . . . . . . . . . . . . . . . . . . . 9

2.3 .1 Reference Clock Counter . . . . . . . . . . . . . . . . . . . . . 9

2.3 .2 Digital Buffer Delay Line . . . . . . . . . . . . . . . . . 10

$2.3 .3 \quad$ Digital Inverter Delay Line . . . . . . . . . . . . . . . . . . . 10

2.4 Third Generation TDC . . . . . . . . . . . . . . . . . . . 11

$2.4 .1 \quad$ Parallel Scaled Delay Elements . . . . . . . . . . . . . . . . . 12

2.4 .2 Vernier TDC $\ldots \ldots \ldots \ldots$

$2.4 .3 \quad$ Local Passive Interpolation (LPI-TDC) . . . . . . . . . . . . . 13

2.5 Background Summary . . . . . . . . . . . . . . . . . . . . . . 16

\begin{tabular}{|lll|}
\hline 3 & ADPLL System Model and Simulation & 17
\end{tabular}

3.1 DCO Model . . . . . . . . . . . . . . . . . . . . . . . . . . . . . . . . 17

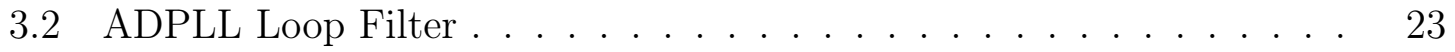

3.3 TDC Model . . . . . . . . . . . . . . . . . . . . . . . . . . . . . . . . . . 24

3.4 Behavioural System Model Demonstration . . . . . . . . . . . . 25

3.5 Summary of the System Model Simulation . . . . . . . . . . . . . . 26

4 TDC Performance Metrics and Specifications 27

4.1 TDC Performance Metrics . . . . . . . . . . . . . . . . . . . . . . 27

$4.1 .1 \quad$ TDC Quantization Error . . . . . . . . . . . . . . . . . . . 29

4.1 .2 Linear Imperfections of a TDC . . . . . . . . . . . . . . . 29

4.1 .3 Non-linear characteristics of a TDC . . . . . . . . . . . . . . . 31

4.1 .4 Single-Shot Precision . . . . . . . . . . . . . . . . 35

4.1 .5 Effective Number of Bits . . . . . . . . . . . . . . . . . . . . 36

4.2 Figure of Merit $\ldots \ldots \ldots$. . . . . . . . . . . . . . . . . . . . . 38

4.3 Deriving the Target Specifications . . . . . . . . . . . . . . . . . . . 39 
4.4 Summary of LPI-TDC Specifications $\ldots \ldots \ldots \ldots$

\begin{tabular}{|lll}
5 & Design of LPI-TDC & 42
\end{tabular}

5.1 Interpolation Delay Cell $\ldots \ldots \ldots \ldots$

5.2 Interpolation Core $\ldots \ldots \ldots \ldots \ldots \ldots \ldots \ldots \ldots$

5.3 Sense Amplifier Assisted Flip-Flop . . . . . . . . . . . . . . . 53

5.4 Signal Buffers $\ldots \ldots \ldots \ldots$. . . . . . . . . . . . . . . . . 60

$5.4 .1 \quad$ Input Buffer $\ldots \ldots \ldots \ldots \ldots \ldots$. . . . . . . . . . . 61

5.4 .2 Interpolator to SAFF Buffer . . . . . . . . . . . . . . 62

5.4 .3 Output Buffer . . . . . . . . . . . . . . . . . . 63

5.5 Top-Level Integration $\ldots \ldots \ldots \ldots$

$5.6 \quad$ Post-Layout Design Verification $\ldots \ldots \ldots \ldots$

5.7 Summary of Simulation Results . . . . . . . . . . . . . . . 67

$\begin{array}{lll}6 & \text { TDC Measured Results } & 70\end{array}$

$6.1 \quad$ Design Evaluation Plan . . . . . . . . . . . . . . . . . . . . . . . 72

6.2 Test bench $\operatorname{Setup~} \ldots \ldots \ldots \ldots \ldots$

6.2 .1 Bench1 Description . . . . . . . . . . . . . . . . . . 74

6.2 .2 Bench2 Description . . . . . . . . . . . . . . . . . 75

$6.2 .3 \quad$ System Noise Measurements _ . . . . . . . . . . . . . . . 76

6.2 .4 Test Bench Summary . . . . . . . . . . . . . . . . 78

6.3 Evaluation PCB Design $\ldots \ldots \ldots \ldots \ldots$

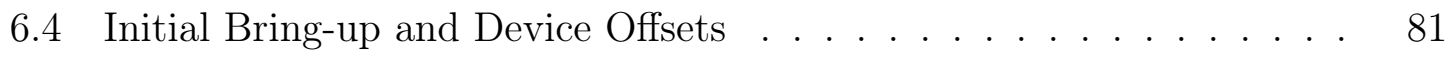

6.5 Maximum Frequency of Operation. . . . . . . . . . . . . . 84

6.6 Correlating Simulation and Measured Results _ . . . . . . . . . . 85

6.6 .1 Investigation Summary . . . . . . . . . . . . . . . . . 87

6.7 Swept Phase Measurement Technique . . . . . . . . . . . . . . . 87 
6.8 SSP Measurements . . . . . . . . . . . . . . . . . . . . . . . . . . . . . . . . 89

$6.8 .1 \quad$ Equipment Noise in SSP Measurements . . . . . . . . . . . . . 90

6.8 .2 Accurate SSP Measurement Summary _. . . . . . . . . . . . 91

6.9 INL, DNL, and Resolution . . . . . . . . . . . . . . . . . . . 93

6.10 Power Consumption . . . . . . . . . . . . . . . . . . . . 94

6.11 Summary of Measurement Results . . . . . . . . . . . . . . 95

\begin{tabular}{|ll|}
\hline 7 Conclusion & 99
\end{tabular}

7.1 Contributions to Research . . . . . . . . . . . . . . . . . . . . . . 99

7.2 Future Work . . . . . . . . . . . . . . . . . . . . . . . . . . 101

\begin{tabular}{ll}
\hline List of References & 103
\end{tabular}

\begin{tabular}{ll}
\hline Appendix A PCB Design Details & 107
\end{tabular}

A.1 Core Card Design . . . . . . . . . . . . . . . . . . . . . . 107

A.1.1 High Speed Input (Start Signal) . . . . . . . . . . . . . . 108

A.1.2 Sampling Clock and Stop Signals . . . . . . . . . . . . . 113

A.1.3 TDC Output Word . . . . . . . . . . . . . . . . . . . . . . . 114

A.1.4 Power Delivery . . . . . . . . . . . . . . . . . . . . 115

A.2 Probe Card Design . . . . . . . . . . . . . . . . . . . 117

A.2.1 Probe Card V1 . . . . . . . . . . . . . . . . . . . . 117

A.2.2 $\quad$ Probe Card V2 . . . . . . . . . . . . . . . . . . . . . . 118

A.3 Board Assembly and Debug . . . . . . . . . . . . . . . . . . . . 119

A.4 Summary of PCB Design . . . . . . . . . . . . . . . . . . 120

\begin{tabular}{|ll|}
\hline Appendix B & 122
\end{tabular} 


\section{List of Tables}

$3.1 \quad$ A theoretical DCO phase noise profile, assumed for the proposed behavioural model. . . . . . . . . . . . . . . . . . . . . 20

3.2 Comparison between desired and modelled DCO phase noise variance. 22

4.1 Cost of manufacturing - academic pricing |1 . . . . . . . . . . . . . . . 39

4.2 List if LPI-TDC specifications for ADPLL application. Several scenarios presented to show the effect of design margin. . . . . . . . . . . . 41

$5.1 \quad$ Differential delay unit (inverter) device sizing. . . . . . . . . . . . . . 48

5.2 Interpolation resistor sizing. . . . . . . . . . . . . . . . . . . 50

5.3 Flip-Flop sampling uncertainty causing bubble errors in TDC output word. . . . . . . . . . . . . . . . . . 54

$5.4 \quad$ Device size specifications for the SAFF. . . . . . . . . . . . . . . . . . 59

5.5 Device sizes of input buffer stages. . . . . . . . . . . . . . . . . . . . 62

5.6 Device sizes of output buffer stages. . . . . . . . . . . . . . . . 64

5.7 List of the LPI-TDC performance metrics based on the extracted postlayout simulations. . . . . . . . . . . . . . . . . . . . . . 69

6.1 Measurements test matrix. . . . . . . . . . . . . . . . . . . . . . . . 72

$6.2 \quad$ List of available lab equipment and designated usage. . . . . . . . . . 73

$6.3 \quad$ Equipment noise measurements for SSP characterization. . . . . . . . 77

6.4 Comparison of Bench1 and Bench2 setups and their preferred usage. $\quad 78$ 
6.5 Logic analyzers available for the LPI-TDC characterization. . . . . . . 79

6.6 PCB variants $\ldots \ldots \ldots \ldots$

$6.7 \quad$ A list of input matching experiments. . . . . . . . . . . . . . . . . . . 85

6.8 Summary of LPI-TDC measured resolution and non-linearity. . . . . . 93

6.9 Measured LPI-TDC performance summary. . . . . . . . . . . . . . . . 98 


\section{List of Figures}

2.1 Simplified timing diagram of a TDC. . . . . . . . . . . . . 5

$2.2 \quad$ Simplified block diagram of an analog phase-lock loop (PLL) $[2] . \ldots . \quad 5$

$2.3 \quad$ ADPLL version where TDC is in place of phase detector $\mid 3] . \ldots .7$

$2.4 \quad$ ADPLL controlled by FCW while TDC assists with fractional error

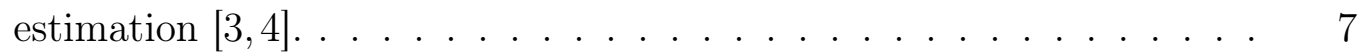

$2.5 \quad$ Digital buffer delay line TDC - a fully digital $2^{\text {nd }}$ generation time-todigital converter $|3| . \ldots \ldots \ldots \ldots \ldots$

$2.6 \quad$ Digital inverter delay line TDC - an improved fully digital $2^{\text {nd }}$ generation LPI-TDC $|3| . \ldots \ldots \ldots \ldots \ldots$

$2.7 \quad$ Dynamic voltage interpolation $|3| . \ldots \ldots \ldots \ldots \ldots \ldots$

$2.8 \quad$ Vernier delay chain LPI-TDC $|3| . \ldots \ldots \ldots \ldots \ldots$

2.9 Local passive interpolation conceptual function $[3] . \ldots \ldots \ldots$

2.10 LPI interpolation conceptual circuit. . . . . . . . . . . . . 15

$3.1 \quad$ ADPLL Simulink model showing the TDC, digital loop filter, and DCO in a feedback configuration. . . . . . . . . . . . . . . . 18

$3.2 \quad$ Typical oscillator phase noise profile $|5| . \ldots \ldots \ldots$

$3.3 \quad$ Limitations of averaging using Egan integration approximation . . . . 20

$3.4 \quad$ Implementation of the DCO noise shaping by means of cascaded filters with different corner frequencies. . . . . . . . . . . . . . . . . 21 
$3.5 \quad$ Open-loop DCO phase noise model compared to desired profile. . . . 21

$3.6 \quad$ ADPLL discrete-time DCO model with phase noise injection. . . . . . 22

$3.7 \quad$ ADPLL DCO model open-loop single side band phase noise spectrum. 23

3.8 TDC model for ADPLL including coarse loop counter and fine phase quantizer. . . . . . . . . . . . . . . . . . 25

3.9 A closed-loop simulation of an ADPLL in Simulink with LBW $=1 \mathrm{MHz}$ $\mathrm{FCW}=50$. . . . . . . . . . . . . . . . . . 26

4.1 Ideal input-output transfer function of TDC. . . . . . . . . . . . . 28

$4.2 \quad$ Linear imperfections causing changes in input-output transfer function characteristic of a TDC. . . . . . . . . . . . . . . . . . . . . . 30

4.3 Integral non-linear imperfections causing changes in input-output transfer function characteristic of TDC. . . . . . . . . . . . . . . . . . 32

4.4 Differential non-linear imperfections causing changes in input-output transfer function characteristic of TDC. . . . . . . . . . . . . . . . . . 32

4.5 Computational principle of INL. . . . . . . . . . . . . . . . . . . 33

$5.1 \quad$ Block level diagram of the proposed LPI-TDC circuit. . . . . . . . . . 42

5.2 TDC differential delay element. . . . . . . . . . . . . . . . 43

5.3 TDC delay cell interpolation principle. . . . . . . . . . . . . . . . 44

5.4 Error of final resistance value of opppcres model compared to rppres for a range of target resistance values and temperature variation. . . . $\quad 45$

5.5 The Inverter Delay Unit schematic showing RF MOSFET transistors. 47

$5.6 \quad$ Inverter delay over VT variation (nominal process corner). . . . . . . 49

5.7 TDC delay unit layout. . . . . . . . . . . . . . . . . . . . . . 50

5.8 Dual delay stage schematic with back-annotated parasitic capacitances and resistances for simulation accuracy. . . . . . . . . . . . . . . . . 51 
5.9 Interpolation core of 5-bit LPI-TDC consisting of 11 individual delay cell units with interpolation factor $\mathrm{IF}=3$ each. . . . . . . . . . . . . . 52

5.10 A basic Flip-Flop block diagram. . . . . . . . . . . . . . . . . . . . 53

5.11 Sense-Amplifier assisted Flip-Flop. . . . . . . . . . . . . . . . . 55

5.12 Sense-Amplifier assisted Flip-Flop. . . . . . . . . . . . . . . . . 56

5.13 Sense-Amplifier common-centroid and inter-digitated layout of input differential pair. . . . . . . . . . . . . . . 56

5.14 SAFF transient behaviour showing conversion sense amplifier in action with small CLK-to-D delay applied at the input. . . . . . . . . . . . 57

$5.15 \mathrm{SAFF} \mathrm{t}_{\text {latch }}$ timing variation due to design offsets caused by device mismatches. . . . . . . . . . . . . . . . . 58

5.16 SAFF CLK-to-Q delay as a function of CLK-to-D delay shows very wide sampling window suitable for LPI-TDC resolution. . . . . . . . . 59

5.17 Cascaded inverters used to drive large capacitive load [2] . . . . . . . 61

5.18 Cascaded inverters used to drive interpolator core. $\mathrm{A} \approx 2.44, \mathrm{~N}=5$ stages. 62

5.19 Cascaded inverters used to isolate interpolator core from the SAFF. $\mathrm{A} \approx 2.44, \mathrm{~N}=3$ stages. . . . . . . . . . . . . . . . . . . . . . . . 63

5.20 ICGCUKIK, LPI-TDC layout with metal fill. . . . . . . . . . . . . 64

5.21 Simulated LPI-TDC interpolator core and system latency. Conditions:

$\mathrm{f}_{\text {start }}=5 \mathrm{GHz}, \mathrm{f}_{\text {stop }}=100 \mathrm{MHz}, \mathrm{VDD}=1.4 \mathrm{~V}, \mathrm{~T}=65^{\circ} \mathrm{C} . \quad \ldots 6$

5.22 Simulated LPI-TDC output code behaviour in response to sweeping test period. Conditions: $\mathrm{f}_{\text {start }}=1 \mathrm{GHz}, \mathrm{f}_{\text {stop }}=100 \mathrm{MHz}, \mathrm{VDD}=1.4 \mathrm{~V}$, $\mathrm{T}=65^{\circ} \mathrm{C} . \ldots \ldots \ldots \ldots$. . . . . . . . . . . . . . . . . . . 67

5.23 Simulated LPI-TDC non-linearity by sweeping test period. Conditions: $\mathrm{f}_{\text {start }}=1 \mathrm{GHz}, \mathrm{f}_{\text {stop }}=100 \mathrm{MHz}, \mathrm{VDD}=1.4 \mathrm{~V}, \mathrm{~T}=65^{\circ} \mathrm{C} . \ldots . . . . \quad 68$

6.1 Photograph of the TDC bare die. . . . . . . . . . . . . . . . . 71 


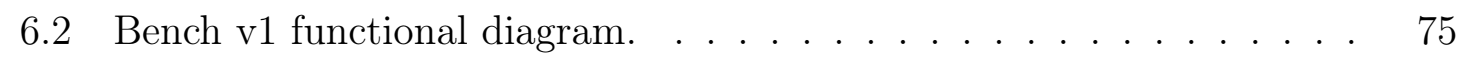

6.3 Bench v2 functional diagram $\ldots \ldots \ldots \ldots \ldots$

6.4 Phase noise of Agilent 81150 measured to quantify its impact on TDC performance . . . . . . . . . . . . . . . . . . 77

6.5 Modular use of test board variants allows for flexible test and debug solution . . . . . . . . . . . . . . . . . 80

$6.6 \quad$ Assembled Core and Probe (v2) cards used in measurements. . . . . 82

$6.7 \quad$ Measured and simulated current consumption as a function of singleended input level voltage. . . . . . . . . . . . . . . . . . 83

$6.8 \quad$ S11 measurements for various board input matching configurations. . 86

6.9 Demonstrating swept phase technique in action to observe LPITDC code transitions for further parametric characterization. . . . . 88

6.10 Demonstrating swept phase technique to assess SSP performance between Bench1 and Bench2 setups. . . . . . . . . . . . . . . . . . 91

6.11 Measured code statistics of core card number 3 under various conditions: $\mathrm{VDD}=(1.4 \mathrm{~V}, 1.2 \mathrm{~V}), \mathrm{f}_{\text {start }}=(1.6 \mathrm{GHz}, 0.8 \mathrm{GHz}), \mathrm{f}_{\text {stop }}=100 \mathrm{MHz} .92$

6.12 Measured LPI-TDC non-linearity at VDD $=1 \mathrm{p} 4 \mathrm{~V}, \mathrm{f}_{\text {start }}=1.6 \mathrm{GHz}$, $\mathrm{f}_{\text {stop }}=100 \mathrm{MHz} \ldots \ldots \ldots \ldots \ldots \ldots \ldots \ldots$

6.13 Measured power consumption at $\mathrm{VDD}=1.4 \mathrm{~V}, \mathrm{f}_{\text {start }}=1.6 \mathrm{GHz}$,

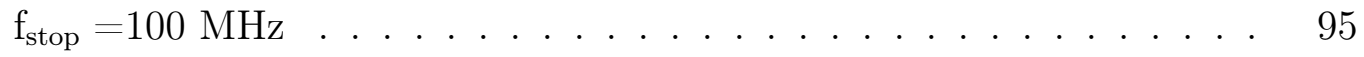

6.14 Measured power and SSP of Core Card number 3 under various conditions: $\mathrm{VDD}=(1.4 \mathrm{~V}, 1.2 \mathrm{~V}), \mathrm{f}_{\text {start }}=(1.6 \mathrm{GHz}, 0.8 \mathrm{GHz}), \mathrm{f}_{\text {stop }}=100$

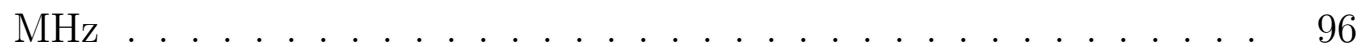

\begin{tabular}{|lll}
\hline A.1 Annotated core card top side view (image from Altium Designer v17) & 108
\end{tabular} A.2 Measuring bond-wire length under the microscope (Leica DVM6A). . 109 
A.3 ICGCUKIK input impedance simulation with updated models based on measured interconnect dimensions. . . . . . . . . . . . . . . . . . . 110

A.4 PCB input matching network with accurate S-parameter models is included in top-level simulation to assess its effectiveness. . . . . . . . 111

A.5 ICGCUKIK input impedance simulation with updated models based on measured interconnect dimensions . . . . . . . . . . . . . . . . . . 111

A.6 Input matching network and high speed start signal path layout . . . 112

A.7 ICGCUKIK LPI-TDC outputs routed in GSG configuration to be

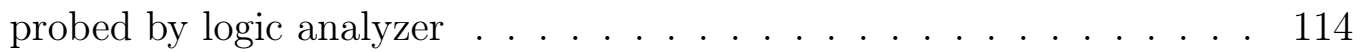

A.8 PCB power planes (darker shade) and top layer tracks (lighter shade) shown with respect to ICGCUKIK and board-to-board connector. . . 115

A.9 Probe card v1 (2x20-pin 0.1" connectors) top side view (image from Altium Designer). . . . . . . . . . . . . . . . . . 117

A.10 Probe card v2 (high density connector) top side view (image from Altium Designer). . . . . . . . . . . . . . . . . . . . . . . . 119

A.11 Re-flow oven setup used to assemble the PCB with delicate surfacemount components. . . . . . . . . . . . . . . . . . . . . . 120 


\section{Nomenclature}

\begin{tabular}{|c|c|}
\hline $\mathrm{AC}$ & Alternating Current \\
\hline $\mathrm{ADC}$ & Analog to Digital Converter \\
\hline BJT & Bipolar Junction Transistor \\
\hline $\mathrm{CMC}$ & Candian Microelectronics Corporation \\
\hline CMOS & Complementary Metal Oxide Semiconductor \\
\hline $\mathrm{DC}$ & Direct Current \\
\hline $\mathrm{DAC}$ & Digital to Analog Converter \\
\hline DNL & Differential Non Linearity \\
\hline DUT & Device Under Test \\
\hline ENIG & Electroless Nickel Immersion Gold \\
\hline ENOB & Effective Number of Bits \\
\hline ESD & Electrostatic Discharge \\
\hline FET & Field Effect Transistor \\
\hline$f_{s}$ & Sampling Frequency \\
\hline$f_{i}$ & Input Frequency \\
\hline HASL & Hot Air Solder Leveling \\
\hline $\mathrm{IC}$ & Integrated Circuit \\
\hline INL & Integral Non Linearity \\
\hline LSB & Least Significant Bit \\
\hline
\end{tabular}




$\begin{array}{ll}\text { MOSFET } & \text { Metal Oxide Field Effect Transistor } \\ \text { MSB } & \text { Most Significant Bit } \\ \text { NFET } & \text { n-Type Field Effect Transistor } \\ \text { NOF } & \text { Number of Fingers } \\ \text { PCB } & \text { Printed Circuit Board } \\ \text { PFET } & \text { p-Type Field Effect Transistor } \\ \text { RF } & \text { Radio Frequency } \\ \text { SMA } & \text { Subminiature Version A Connector } \\ \text { SNR } & \text { Signal to Noise Ratio } \\ \text { TDC } & \text { Time to Digital Converter } \\ \text { VDD } & \text { Positive Supply Voltage (Also known as V } \text { ) } \\ \text { VEE } & \text { Negative Supply Voltage (Also know as VSS) }\end{array}$




\section{Chapter 1}

\section{Introduction}

\subsection{Motivation and Objectives}

The growing rate of information exchange requires efficient ways to manage data storage and throughput. Phase-locked loops (PLL) have been used by engineers for many decades in data recovery, re-timing, and jitter termination. Although analog solutions continue to push the performance boundary, the digitally-assisted and all-digital PLL (ADPLL) implementations can provide competitive alternatives to traditional analog PLL designs in many applications. Some of the ADPLL architectures utilize time-to-digital converters (TDC) in place of traditional phase detectors. However, prior to usage in ADPLL, TDC were commonly used in other applications where the relative time interval between events had to be determined, such as radars, high-energy particle physics, lasers, 3D imaging etc. An ideal TDC would be able to resolve infinitely-small time intervals (resolution), consume no energy, have infi-

nite dynamic range (maximum detectable time interval), while maintaining perfect linearity. However, real-world limitations of modern technologies set practical limits to these properties usually many orders of magnitude greater than Planck's energy and time. Therefore, it often comes down to a design choice to trade off various 
performance parameters, depending on application requirements. In a ADPLL, the loop components are usually desired to operate at high rates for better performance and reduced design complexity, while staying within the power budget. In return, the system could potentially achieve a smaller footprint and cost, which is an important consideration for a successful design. The semiconductor manufacturing industry has been continually increasing transistor density for digital data storage to reduce large volume production costs. This led to appearance of many silicon-based ( $\mathrm{Si}$ ) complimentary metal oxide semiconductor (CMOS) process technology nodes and flavours, which inherently favour a digital design methodology (i.e. for device placement and modelling). Therefore, there are now many CMOS Si process options available that allow designers to find optimal balance between cost and design effort for required performance.

The goal of this work is to explore the design and implementation of a timeto-digital converter based on the Local Passive Interpolation principle which can directly quantize a $5 \mathrm{GHz}$ input signal, making it the fastest in the recently reported literature. The inherent design simplicity and monotonicity makes it an attractive architectural choice for high-speed TDC applications, which also helps avoid some of the calibration challenges. A high frequency of operation could simplify the overall system complexity as fewer elements are required to close the feedback loop. The LPI-TDC also exhibits a very low conversion latency, thus making it capable of sequentially quantizing an input signal at the rates exceeding $100 \mathrm{MHz}$. Moreover, this time-to-digital converter is implemented in a matured low-cost $0.13 \mu \mathrm{m}$ CMOS process to reduce the design cost. For the TDC to be useful in modern ADPLL applications, a fine resolution of 8ps is targeted for a conceptual demonstration. 


\subsection{Contributions}

An architecture based on the local passive interpolation (LPI) was selected to achieve a good inherent converter linearity. The LPI-TDC was fabricated and measured to demonstrate the feasibility of this design, capable of successfully converting a $1.7 \mathrm{GHz}$ signal at $100 \mathrm{MHz}$ rate. The resolution of $8.1 \mathrm{ps}$ and good linearity were achieved, closely matching the simulation results. A thorough investigation was also performed to conclude that the proposed design is capable of converting signals at frequencies close to the targeted $5 \mathrm{GHz}$ with careful attention to signal integrity and power supply impedance.

\subsection{Thesis Organization}

This work begins with background information presenting various TDC architectures in Chapter 2. A conceptual yet highly-accurate behavioural model of ADPLL that uses time-to-digital converter is examined in Chapter 3 Performance trade-offs and choice of specifications for this work are derived and presented in Chapter 4 Chapter 5 focuses on LPI-TDC block-level design including schematic, layout, and simulation results. Measured results are then presented in Chapter 6 along with a discussion of the interpretation in light of targeted specifications. Chapter 7 concludes this work, with the research contributions and suggestions for further design improvements of the LPI-TDC. 


\section{Chapter 2}

\section{Background}

In this chapter the ADPLL application is presented in Section 2.1. One of the components used in ADPLL is a time-to-digital converter (TDC) circuit.

The TDC quantizes a time interval $\mathrm{T}_{\text {test }}$ between rising edges of the start and stop signals into a discrete digital code (word, $\mathrm{B}_{\text {out }}$ ) as shown in Figure 2.1 Various time-to-digital converter architectures are discussed in Section 2.2. Section 2.3 Section 2.4, showing a gradual evolution from the early designs to the modern generation.

\section{$2.1 \quad$ ADPLL Background}

One of the key components in most modern electronic systems is a Phase-Locked Loop (PLL). It is a closed feedback system, which establishes a desired frequency and phase relationship between output and an input reference signal. Through careful selection of feedback parameters, the output of the PLL can track, scale, and clean up the noise of the reference signal. Such functionality is crucial in many applications including high speed data transfer and RF systems. The analog implementation of a PLL was achieved well before the modern age of digital electronics. However, a digital equivalent has been explored in recent years, and has shown viable alternatives 


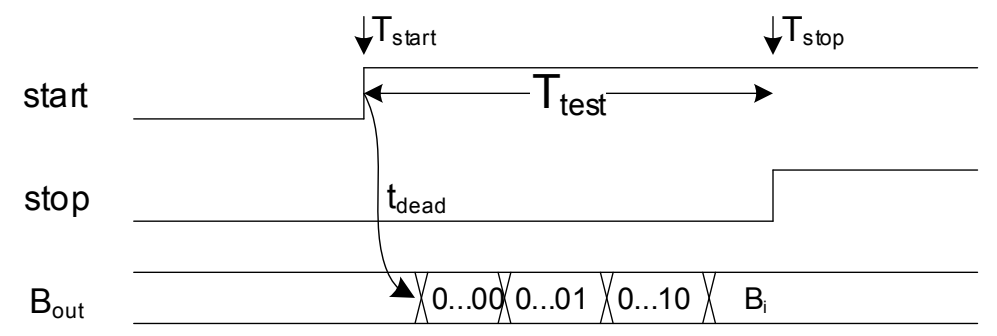

Figure 2.1: Simplified timing diagram of a TDC.

to analog counterparts in some applications, achieving a high degree of integration.

\subsubsection{Analog PLL Solution}

The key elements of a traditional analog PLL are voltage-controlled oscillator (VCO), phase-frequency detector (PFD), charge pump, loop filter (LPF), and dividers which set desired frequency scaling. One of the classical architectures is presented in Figure 2.2. While most of the components in an analog PLL circuit can be easily inte-

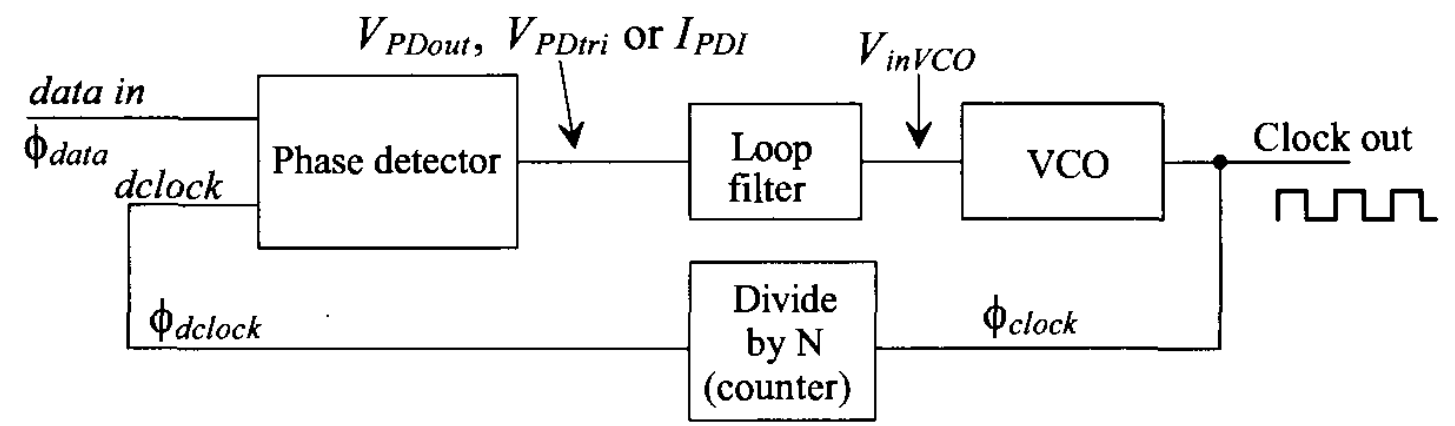

Figure 2.2: Simplified block diagram of an analog phase-lock loop (PLL) 22.

grated on chip, the loop filter and charge pump passive components often remains a 
challenge and often have to be placed off-chip. Very high performance can be achieved using an analog implementation mainly at the expense of area and power which is required to overcome the dropping ratio of transistor transconductance to output conductance $\left(\frac{g m}{g d s}\right)$ in modern fabrication processes 3 .

\subsubsection{All Digital PLL Solution}

An alternative solution has been proposed in recent years, which takes aim at replacing the analog filter with a digital equivalent (IIR, FIR). Such choice of feedback conditioning requires a change to digital signalling instead of traditional analog lowswing or high-sensitivity type. This also takes advantage of emerging fabrication processes catered towards digital signalling. Using digital techniques can also enable the use of older CMOS technologies, thus allowing some applications to save cost for a given performance. The research towards an all-digital PLL solution has yielded several architectural choices adopted in many practical designs.

A typical ADPLL has the following elements to form the feedback loop: digitallycontrolled oscillator (DCO), phase quantizer, digital loop filter, and feedback dividers. It should be further noted that a phase quantizer typically consists of an integer

counter and time-to-digital converter (TDC). The two sub-elements provide coarse and fine (fractional) digital encoding of the number of periods produced by the DCO.

There are two ADPLL architectures that can be commonly found in the reference literature 3. 4]. One of the early ADPLL architectures used a TDC directly in place of a phase-frequency detector as seen in Figure 2.3 [3]. Therefore, just like in a classical PLL, the output frequency of the DCO is determined by the input reference signal and the feedback divider ratio that scales it. The system is conceptually simple to implement with few components and only the DCO and feedback divider have 


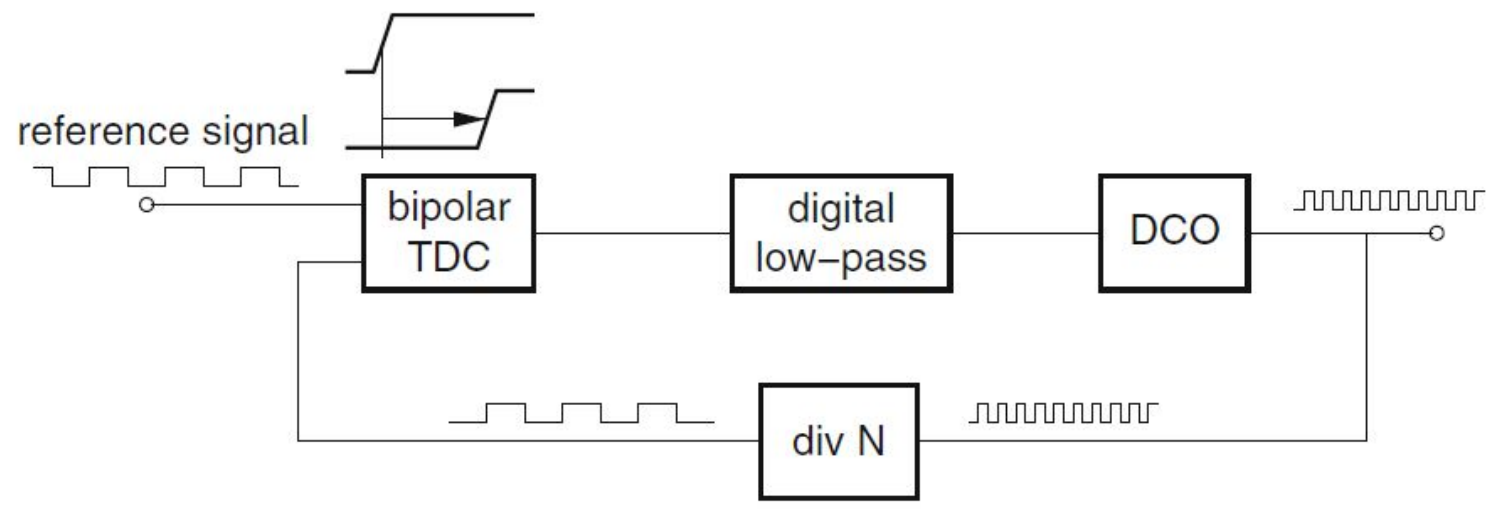

Figure 2.3: ADPLL version where TDC is in place of phase detector 3 .

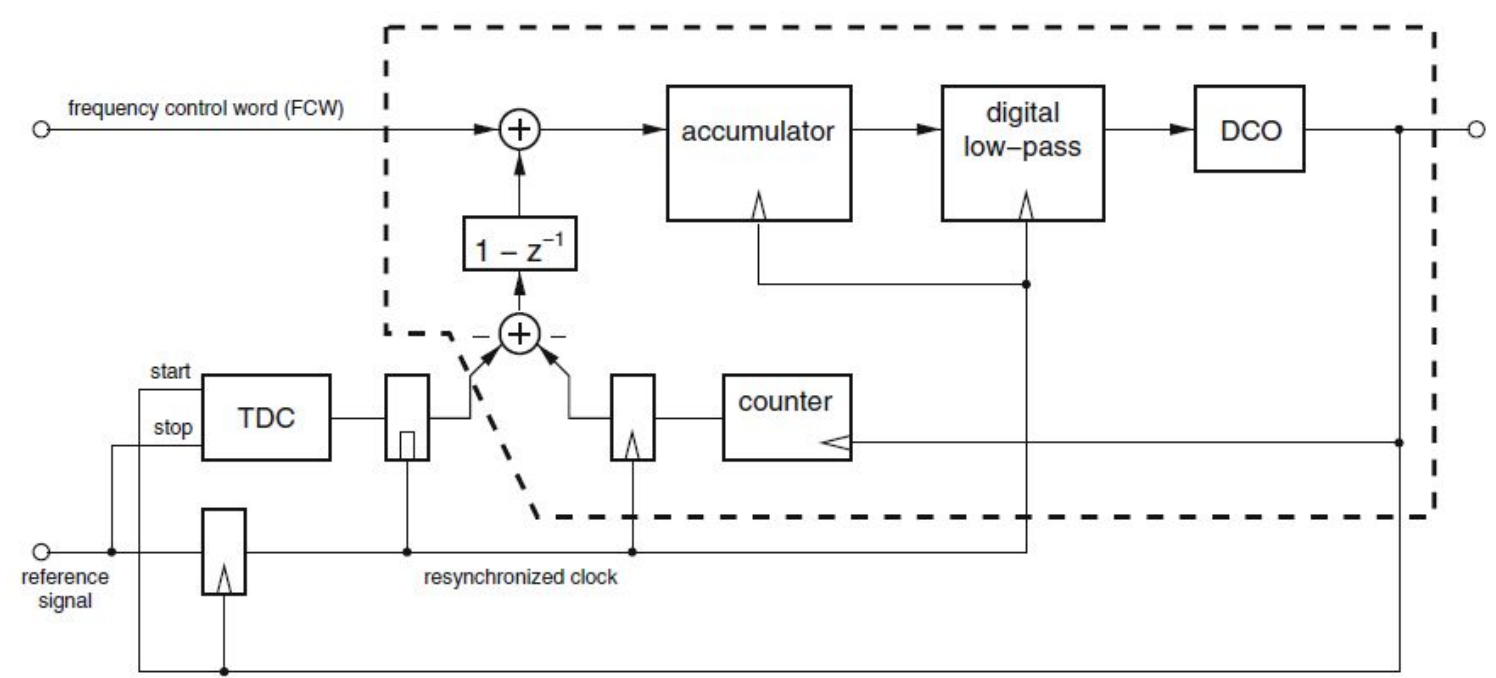

Figure 2.4: ADPLL controlled by FCW while TDC assists with fractional error estimation [3,4].

to handle a high frequency of operation. However, the feedback divider introduces additional noise and effectively reduces the loop gain, thus causing some performance degradation [6].

An alternative solution is to place the phase quantizer directly at the output of DCO as shown in Figure 2.4 The digital code could then be compared to a frequency control word (FCW), which sets the target for desired DCO output frequency. As a result, the feedback divider can be eliminated entirely. However, the overall ADPLL 
noise performance becomes more dependent on the TDC design. Such architectures could yield reduced chip area and possibly reduced power depending on the ADPLL application.

\subsection{First Generation TDC}

Some of the first designs involved converting time difference into voltage, followed by an analog-to-digital converter (ADC) to encode it. Such architecture has many sensitive analog components, which limit the process scalability.

\subsubsection{Single Integrator Design}

The start and stop signal generate a pulse, whose width is equivalent to the phase difference between the two signals, i.e. the test time interval $T_{\text {test }}$. Using a charge pump, the voltage pulse is converted to current that is integrated by a capacitor at

a rate of $\frac{d V}{d T}=\frac{I_{1}}{C_{1}}$, reaching a final value of $V_{1}=\frac{I_{1}}{C_{1} T_{\text {test }}}$. The resulting voltage $V_{1}$ is proportional to the original phase difference between the start and stop signals. The process, voltage, and temperature (PVT) variation will affect the integrator function as charge pump current and capacitance may vary, thus a calibration is required.

\subsubsection{Dual-slope Integrator Design}

A solution based on dual-slope integration, that is commonly used in an ADC, can be applied in order to reduce the error and possibly remove the need for calibration. At the arrival of the start signal, a counter is set off at a rate of $f_{r e f}$. At the same instance, the first integration initiates until the arrival of the stop signal at a rate of $\frac{d V}{d T}=\frac{I_{1}}{C_{1}}$, reaching the final value of $V_{1}=\frac{I_{1}}{C_{1} T_{\text {test }}}$. The second integration begins at the arrival of stop signal and continues until the voltage reaches the $V_{1}$ level but at 
a slower rate of $\frac{d V}{d T}=\frac{I_{2}}{C_{2}}$. Once the two voltages are equal, the counter is stopped. The measurement period can now be estimated as $T_{\text {test }}=\frac{\text { count }}{f_{\text {ref }}\left(1+\frac{C_{2}}{C_{1}} \cdot \frac{I_{1}}{I_{2}}\right)}$. The part of the error due to process variation is greatly reduced by the reliance on capacitor and current ratios instead of the absolute values. The non-ideal comparator offsets and charge pump linearity can potentially degrade the conversion error. Moreover, the variation due to voltage and temperature (VT) may require calibration to achieve better accuracy. The inherent analog nature of the first generation TDC does not allow for easy process scalability, as each component would have to be carefully designed for each technology. The conversion speed is also generally low due to large system latencies, which is especially apparent in the case of a dual-slope TDC due to the effective time amplification function. The resolution is limited to one period of the counter reference frequency $f_{\text {ref }}$.

\subsection{Second Generation Digital TDC}

A fully-digital solution is required to enable process scalability and design portability. This is achieved by removing the analog transformation step and instead, adopting a technique that implements a direct time conversion into digital data. The resolution of the all-digital second generation time-to-digital converter is limited to an inverter delay for a given technology.

\subsubsection{Reference Clock Counter}

The simplest method is by using a high frequency reference clock as a counter, where a time interval is measured by counting the number of cycles that occur during its duration.

But in order to achieve a high resolution it is necessary to generate a very high 
clock frequency, which has its own limitations in terms of power consumption and counter timing restrictions. These problems can be solved by introducing a TDC that will do an interpolation. In other words, it will perform a more precise subdivision of one clock period into several intervals using the same generator oscillator to obtain different phases of the reference clock.

\subsubsection{Digital Buffer Delay Line}

When a more precise resolution is required it is possible to obtain it by using digital elements to create a linear chain of delay for the reference clock as shown in Figure 2.5

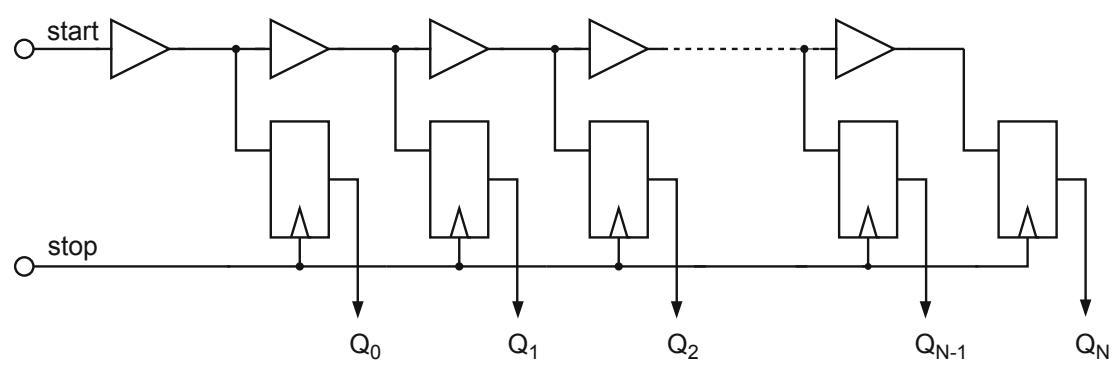

Figure 2.5: Digital buffer delay line TDC - a fully digital $2^{\text {nd }}$ generation time-todigital converter 3 .

In this case, the clock acts as a start signal that goes through the delay buffers, whose outputs are connected to flip-flops or latches that will sample the different states of the clock when the rising edge of the stop signal occurs; the result of this sampling will generate a thermometer code.

\subsubsection{Digital Inverter Delay Line}

To further improve digital delay line resolution, an inverter is used in place of a buffer (Figure 2.6), since the delay of a CMOS inverter is only $\frac{1}{2}$ of the delay of a buffer. 
In this case, the rising and falling progressions are used for measurement, the phase

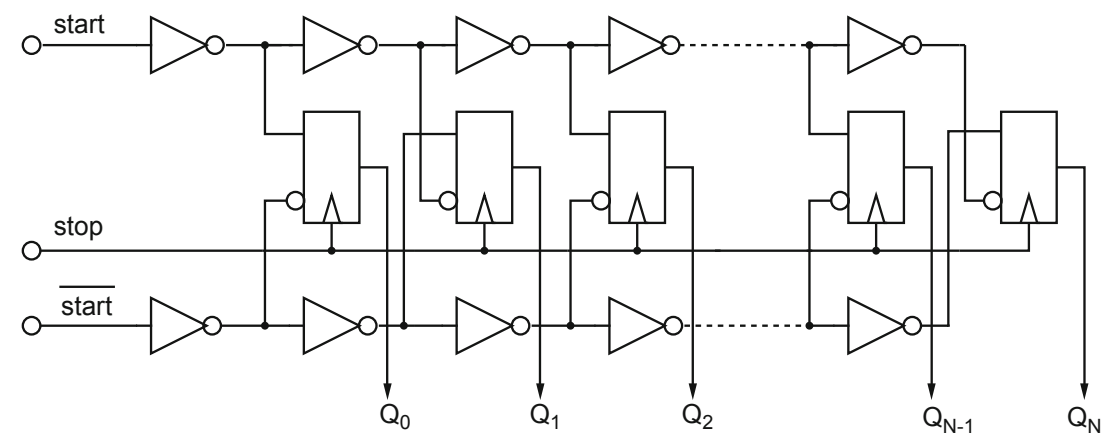

Figure 2.6: Digital inverter delay line TDC - an improved fully digital $2^{\text {nd }}$ generation LPI-TDC [3].

change of the alternation from high to low sequence will determine the time interval length.

Two major inconveniences with this TDC are the asymmetric delay between rising and falling transitions of the inverter as well as technology limitations in terms of resolution.

\subsection{Third Generation TDC}

The third generation of time-to-digital converters is marked by achieving sub-gate delay resolution in an all-digital implementation. For this discussion, an inverter propagation delay with output fan-out of 2 (FO2) defines a single gate delay $t_{d}^{\text {gate }}$ because it represents a somewhat standardized practical limit for a given CMOS technology. Therefore, a TDC resolution $\mathrm{T}_{\mathrm{LSB}}$ less than a gate delay is typically achieved through clever circuit design techniques. To help further this discussion, an interpolation factor is introduced, which simply refers to a ratio of TDC resolution to $t_{d}^{\text {gate }}$ propagation 
delay $((2.1))$.

$$
I F=\frac{t_{d}^{\text {gate }}}{T_{L S B}}
$$

\subsubsection{Parallel Scaled Delay Elements}

The sub-gate resolution is achieved by using capacitive elements to delay the start signal at different rates (i.e. $\mathrm{RC}$ delay) Figure 2.7 The stop signal samples all

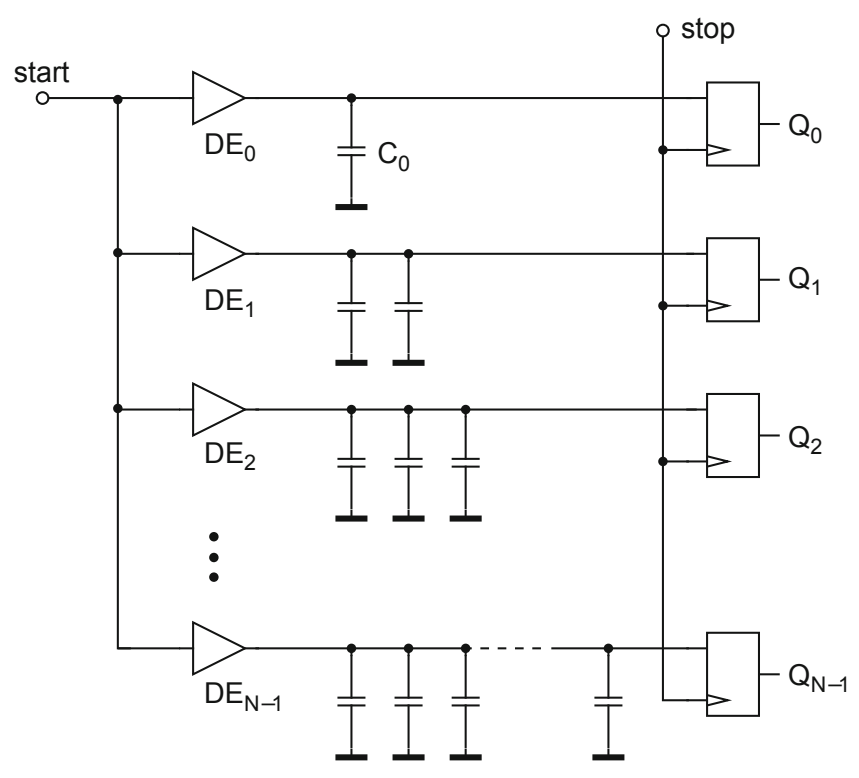

Figure 2.7: Dynamic voltage interpolation [3].

latches in parallel. Although it is feasible to achieve fairly small delay, it is difficult to control it over PVT variation. Moreover, the current silicon CMOS technologies are not very well suited to implement large capacitors on-chip (e.g. to extend dynamic range), thereby, making this choice fairly area-inefficient.

\subsubsection{Vernier TDC}

This method has been one of the most popular methods of implementing a sub-gate resolution TDC 17,8. The basic method relies on the ratio of propagation delays 
between two inverter delay chains Figure 2.8. This method can achieve low resolu-

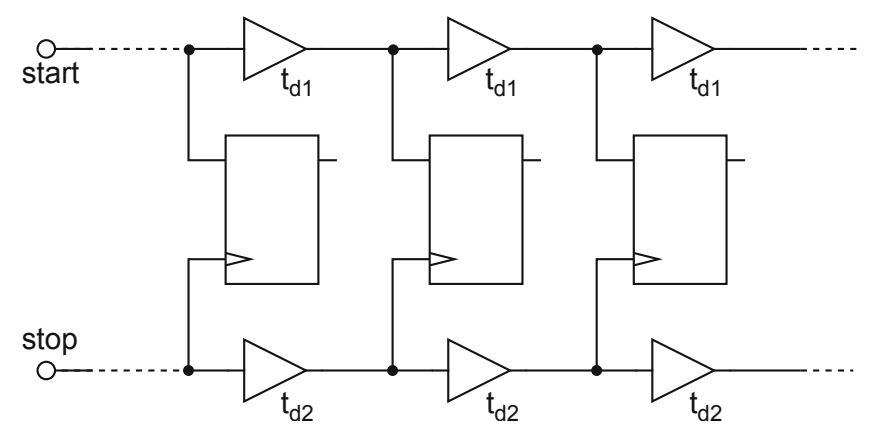

Figure 2.8: Vernier delay chain LPI-TDC 3].

tion. However, the number of active delay stages grows linearly with dynamic range. Maintaining well-controlled delay ratios requires complex calibration due to inherent susceptibility to PVT variation and mismatch, which results in design overhead.

\subsubsection{Local Passive Interpolation (LPI-TDC)}

The LPI-TDC main principle of operation is similar to that of the delay line architecture. However, in order to achieve sub-gate delay resolution, a voltage interpolation technique is used instead of vernier-style ratio of two delay elements. The principle is depicted in Figure 2.9. A rising edge $\mathrm{V}_{\mathrm{A}}$ (e.g. start signal) at time $\mathrm{t}_{\mathrm{A}}$ is delayed by $t_{d}$ of a single delay element, thus producing another output rising edge $V_{B}$ arriving at time $t_{\mathrm{B}}$. The two edges appear exactly $t_{d}^{\text {gate }}=t_{A}-t_{B}$ apart. Applying the input $\left(\mathrm{V}_{\mathrm{A}}\right)$ and output $\left(\mathrm{V}_{\mathrm{B}}\right)$ to a simple voltage divider, yields a derived output $\left(\mathrm{V}_{\mathrm{int}, \mathrm{i}}\right)$ that has a voltage level defined by 2.2 .

$$
V_{\text {int }, i}=V_{B}+a_{i}\left(V_{A}-V_{B}\right)
$$




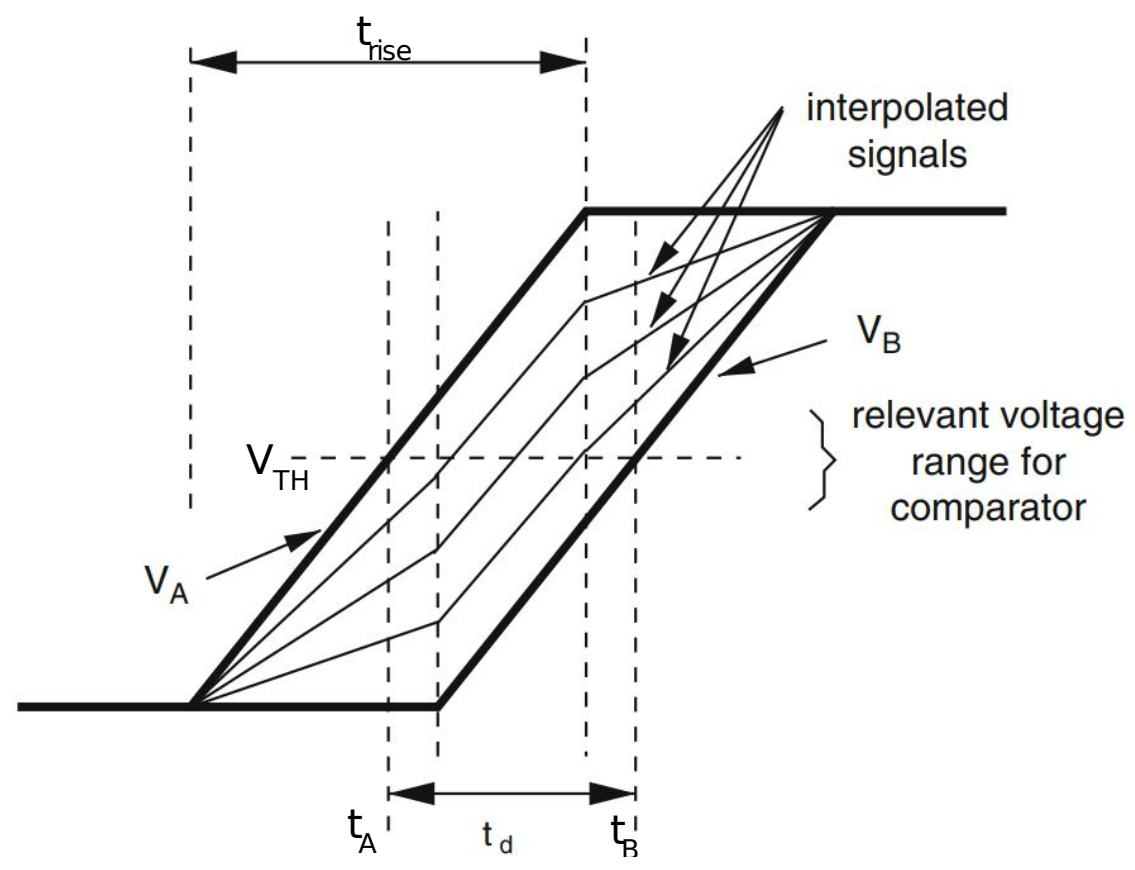

Figure 2.9: Local passive interpolation conceptual function 3 .

The coefficient $a_{i}=\frac{i}{I F}$ essentially represents voltage divider ratio at a particular node, and also determines how far away the interpolated signal $V_{\text {int,i }}$ will appear from the input $V_{A}$. The subscript $i$ implies that there could be multiple voltage divider outputs.

When both signals $V_{A}$ and $V_{B}$ are switching (with the same polarity), the interpolated outputs $\mathrm{V}_{\text {int,i }}$ should look like $\mathrm{V}_{\mathrm{A}}$ shifted linearly in time, which ideally would happen around threshold mid-level $\mathrm{V}_{\mathrm{TH}}$. Conceptually, each interpolated signal $\mathrm{V}_{\text {int,i }}$ would arrive after signal $\mathrm{V}_{\mathrm{A}}$ at a time $t_{i}=t_{A}+t_{d} \cdot a_{i}$. Sampling all interpolated signals with a common stop signal completes the TDC action and produces a pseudo-thermometer code, thus revealing the phase information between the start and stop signals. A conceptual circuit representation linearized around $\mathrm{V}_{\mathrm{TH}}$ is shown in Figure 2.10. The LPI-TDC resolution is $T_{L S B}=\frac{t_{d}^{\text {gate }}}{I F}$, which scales linearly with number of interpolation nodes per delay element. 


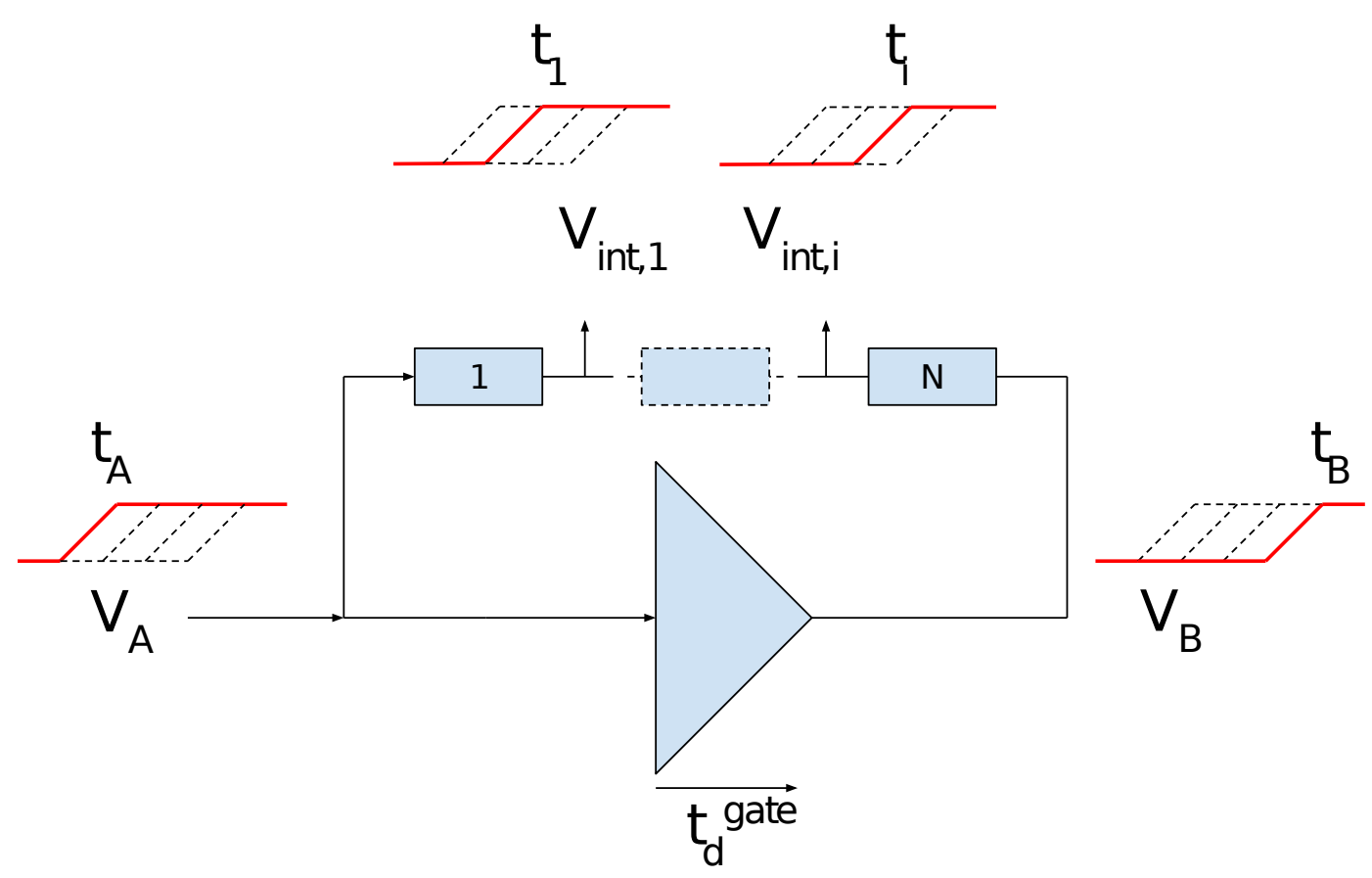

Figure 2.10: LPI interpolation conceptual circuit.

One of the main design challenges is to ensure that the voltage divider (passive interpolator) operates much faster than $t_{d}^{\text {gate }}$ delay to ensure that the interpolated signals $\mathrm{V}_{\text {int,i }}$ track the voltage changes $\left(V_{A}-V_{B}\right)$ in a linear fashion. Another challenge is to create an inverter delay element that has the same polarity at input and output. Further design details are discussed in Chapter 5. Some of the biggest advantages of LPI-TDC are low latency, good inherent linearity (with appropriate design techniques), sub-gate resolution while using lower number of active stages compared to Vernier style delay lines, which makes it a great candidate for fast ADPLL applications. 


\subsection{Background Summary}

The challenges of the analog PLL solutions were discussed to show some of the advantages the All-Digital PLL (ADPLL) alternatives have to offer. One of the key components in a modern ADPLL is a phase quantizer. A time-to-digital converter (TDC) was introduced as a common implementation of such quantizer. Several TDC generations have been presented, starting with a discussion of the analogcentric architectures in the early designs (first generation) and finishing with a third generation of digital implementation that take advantage of modern scalable CMOS processes. A local passive interpolation technique has also been presented, and will be discussed in further detail in this work. The discussion has mostly been focused on the core TDC designs, since most architectures can be arranged in a loop configuration (Nutt method) to extend the effective dynamic range of a phase quantizer [9]. 


\section{Chapter 3}

\section{ADPLL System Model and Simulation}

This chapter describes a discrete-time ADPLL system model that was created in MATLAB using Simulink toolkit. The purpose of this simulation is to demonstrate the loop dynamics when TDC is included in the feedback. A simplified architecture of the ADPLL shown in Figure 3.1 has been constructed. The following key elements have been modelled Figure 3.1: TDC, digitally-controlled oscillator (DCO), loop filter to control the closed-loop behaviour. A more sophisticated model could also include behavioural impairments such as non-linearity, noise, and metastability [4]. The ADPLL loop tracks the digital frequency control word (FCW) to generate a desired output frequency at the output of the DCO.

\subsection{DCO Model}

The DCO model was implemented with great attention to accuracy. It includes such features as DCO gain $\left(\mathrm{k}_{\mathrm{DCO}}\right)$, free-running frequency $\mathrm{f}_{0}$, and an accurate phase noise behavioural model. A novel method of DCO flicker noise modelling is proposed. Similar to some other works, the DCO noise shaping is based on the principle of filtering an Additive White Gaussian Noise source (AWGN) 4, 10, 11. 


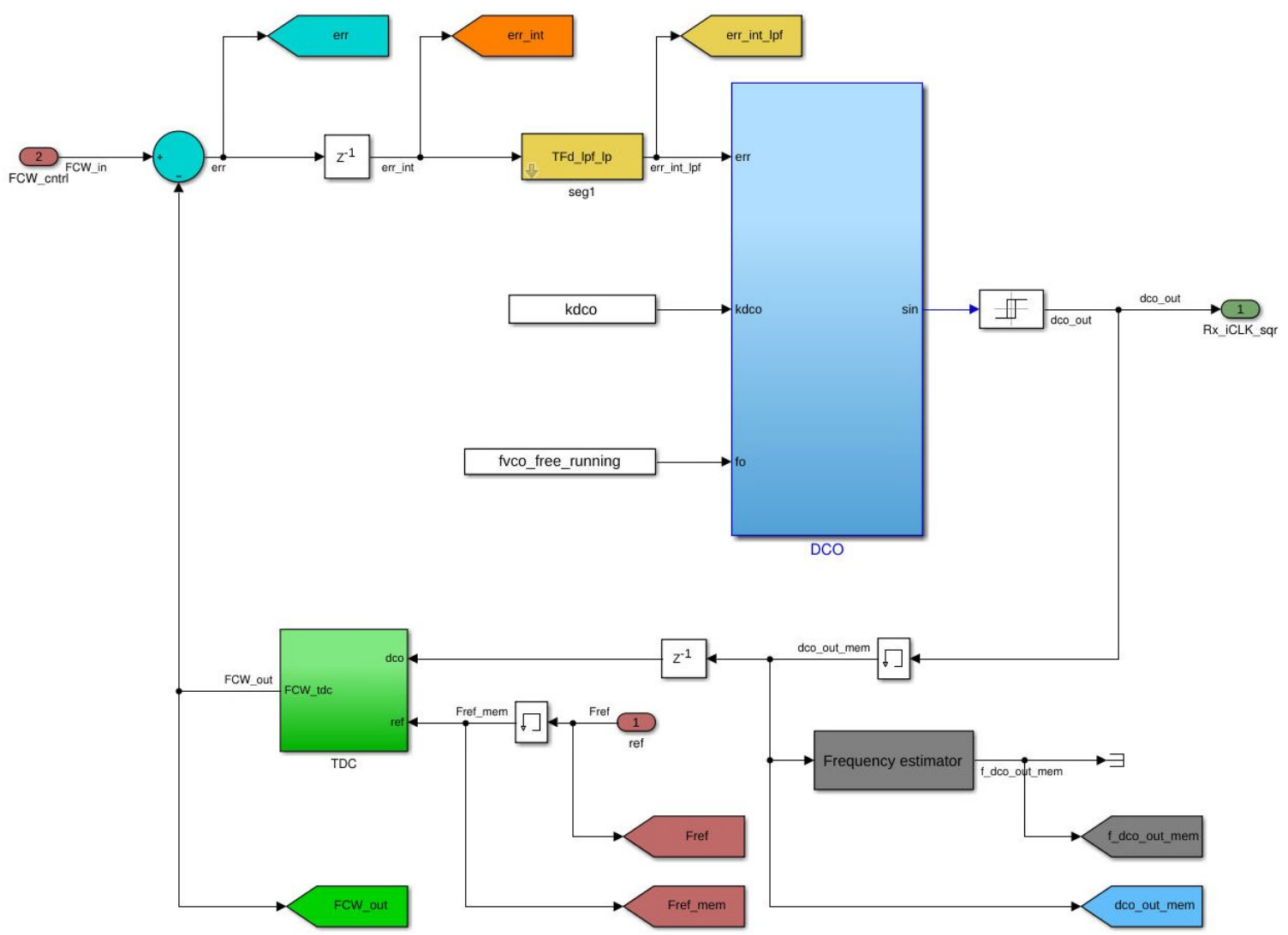

Figure 3.1: ADPLL Simulink model showing the TDC, digital loop filter, and DCO in a feedback configuration.

However, unlike a fixed slope piece-wise-linear [10], parallel low-pass filter [4], or subsampling model [11], this work is proposing to use the band-pass filter approach. Such method allows for great accuracy while maintaining low implementation complexity and flexibility.

A phase noise profile for an oscillator can be represented by a generalized shape shown in Figure 3.2. A good explanation to physical phenomenon causing the particular noise shape is discussed in [5].

Integrating the phase noise power spectral density (PSD) yields phase variance in $\operatorname{rad}^{2}$ (i.e. time domain jitter). The goal of the behavioural time-discrete model is to 


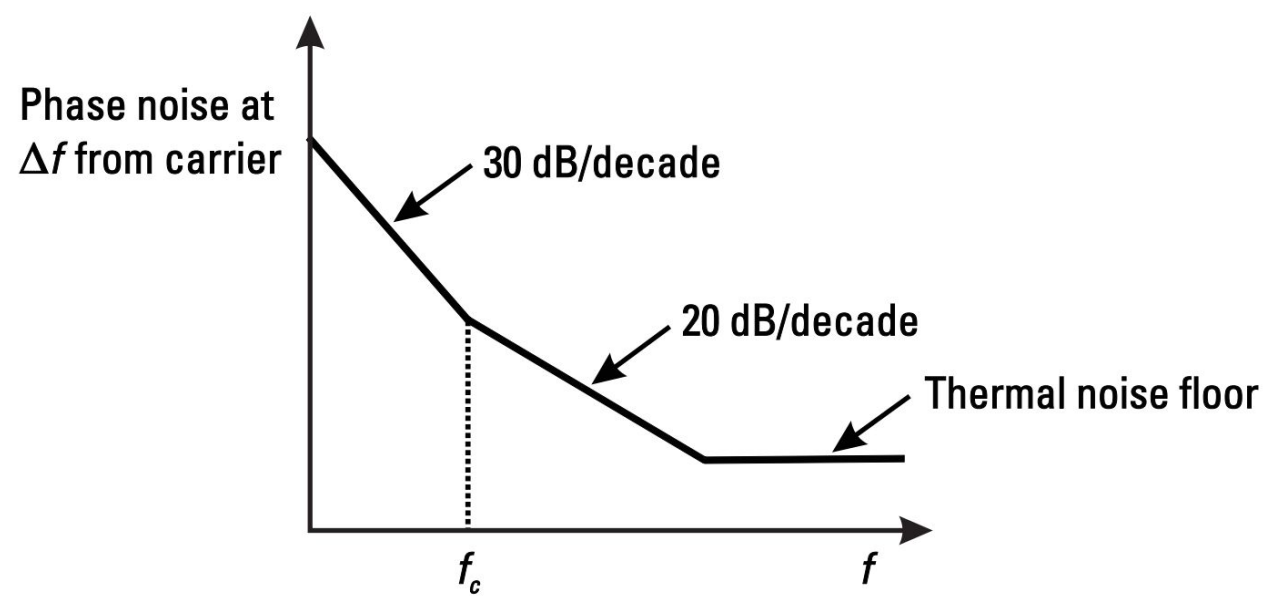

Figure 3.2: Typical oscillator phase noise profile [5].

closely match the phase noise variance of an actual DCO. The first step of approximation is realizing that the phase noise profile can be segmented into a piece-wise linear representation. The more segments there are the more complex and accurate the model would be required to be in order to achieve a perfect match. Passing the AWGN source through a combination of band-pass filters (one for each linear segment), it is feasible to approximate the phase noise variance with high degree of accuracy. Using an iterative approach, a set of custom-order band-pass filters was designed for each segment to match individual contribution to the total phase variance.

To reduce the computational complexity, a simplified integration technique was used, proposed by William F. Egan in [12]. For a given log-log PSD segment, which has phase noise PSD level $S\left(f_{1}\right)$ at starting frequency $f_{1}$ and $S\left(f_{2}\right)$ at ending frequency $\mathrm{f}_{2}$, the phase noise power can be estimated by a set of equations given in 3.1 and (3.2).

$$
\text { slope } b=\frac{S\left(f_{1}\right)-S\left(f_{2}\right)}{10 \cdot \log _{10}\left(\frac{f_{2}}{f_{1}}\right)}
$$




$$
\left.P_{y}\right|_{f 2} ^{f 1}= \begin{cases}\frac{f_{1} S_{y}\left(f_{1}\right)}{b+1} \cdot\left[\left(\frac{f_{2}}{f_{1}}\right)^{b+1}-1\right], & \text { if } b \leq-1 \\ f_{1} \cdot S_{y}\left(f_{1}\right) \cdot \ln \left(\frac{f_{2}}{f_{1}}\right), & \text { otherwise }\end{cases}
$$

The integration accuracy is a function of slope as shown in Figure 3.3

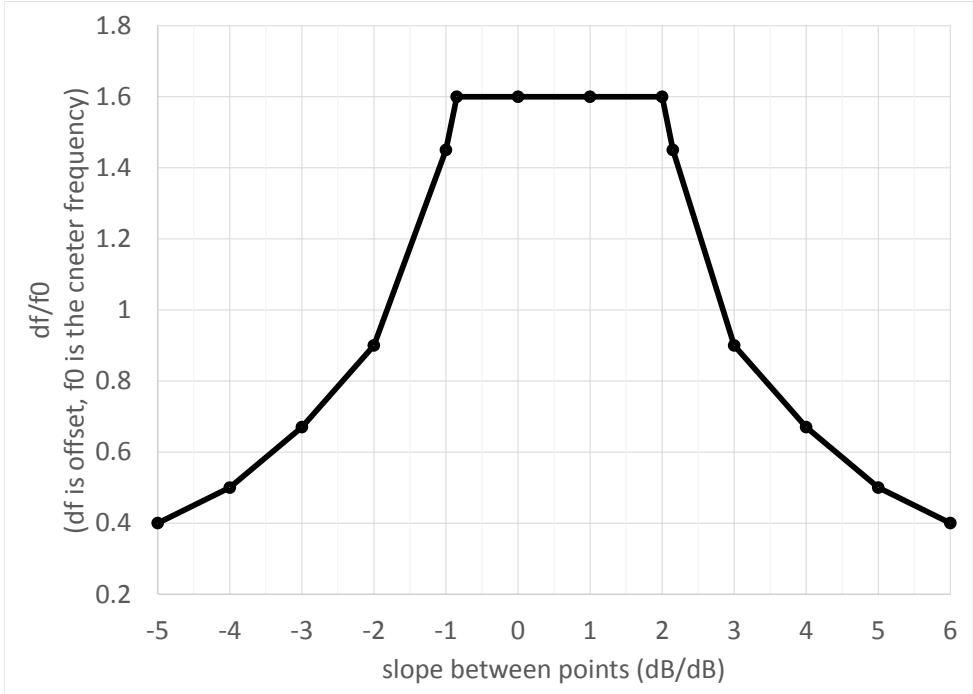

Figure 3.3: Averaging range $\left(\frac{\Delta f}{f 0}\right)$ that causes $1 \mathrm{~dB}$ error using Egan integration approximation $[12$.

A simple 2-segment phase noise profile was assumed as shown in Table 3.1

Table 3.1: A theoretical DCO phase noise profile, assumed for the proposed behavioural model.

\begin{tabular}{lc}
\hline $\mathrm{f}(\mathrm{Hz})$ & $\mathrm{S}_{\mathrm{f}}(\mathrm{dBc} / \mathrm{Hz})$ \\
\hline $10 \mathrm{k}$ & -43 \\
$500 \mathrm{k}$ & -94 \\
$1 \mathrm{G}$ & -160 \\
\hline
\end{tabular}

The two segments have been approximated using cascaded low-pass and high-pass filters placed after a AWGN source to yield the same total noise power (Figure 3.4 
and Figure 3.5). It should be noted, that the model accuracy can be further improved by segmenting the phase noise profile into narrower bands.

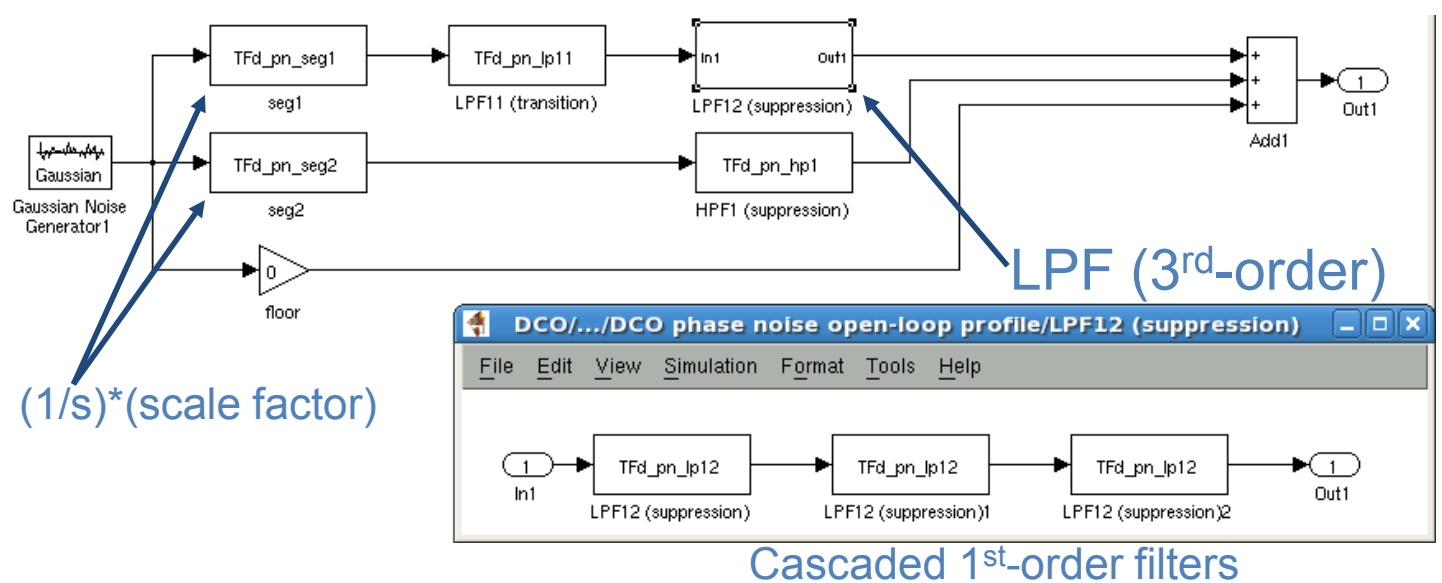

Figure 3.4: Implementation of the DCO noise shaping by means of cascaded filters with different corner frequencies.
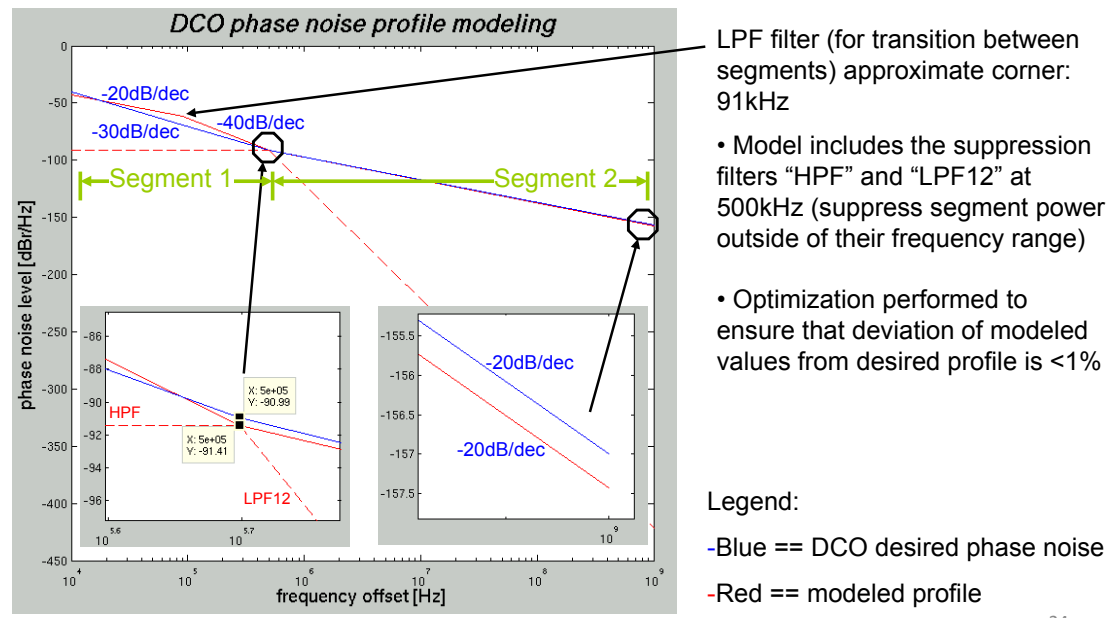

Figure 3.5: Open-loop DCO phase noise model compared to desired profile.

The integrated phase noise power of the simple profile and noise variance provided by the piecewise-linear band-pass model is summarized in Table 3.2

The noise power for individual and the overall variance is $« 1 \%$ between the desired 
Table 3.2: Comparison between desired and modelled DCO phase noise variance.

\begin{tabular}{ccc}
\hline segment & desired varaince (Egan integration) & band-pass model variance \\
\hline 1 & 0.50053 & 0.50051 \\
2 & 0.00039816 & 0.00040099 \\
total & 0.50093 & 0.50091 \\
\hline
\end{tabular}

profile and the equivalent model. The DCO noise is then added to the discrete time (phase) domain DCO model shown in Figure 3.6. The model was verified through simulation in an open-loop configuration, showing an acceptable match with expected phase noise (Figure 3.7).
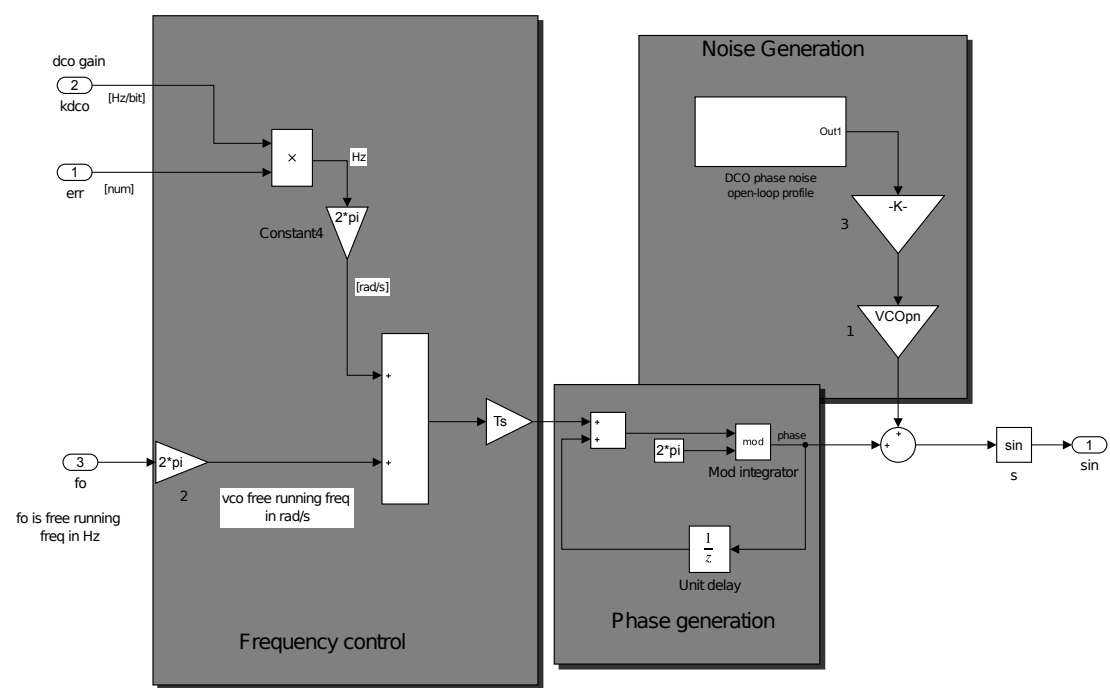

Figure 3.6: ADPLL discrete-time DCO model with phase noise injection. 


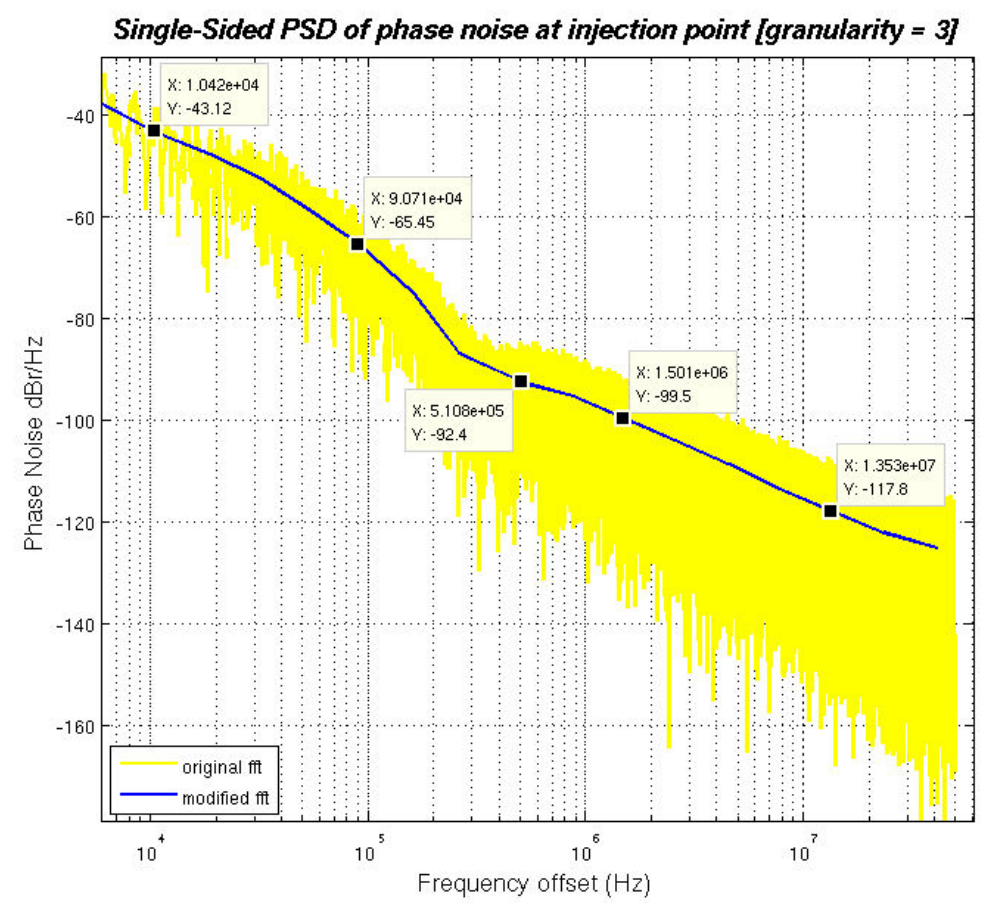

Figure 3.7: ADPLL DCO model open-loop single side band phase noise spectrum.

\subsection{ADPLL Loop Filter}

To control the ADPLL feedback path dynamic behaviour (response and stability), an ideal discrete-time domain filter was implemented, which sets the overall loop bandwidth (LBW). An s-domain filter was defined and then passed through a built-in MATLAB tool $c 2 d$ to complete the conversion from the continuous to the discretetime domain. 


$$
\begin{aligned}
f_{\text {loop }} & =1 M H z \quad(L B W) \\
T_{s} & =1 p s \quad(\text { discrete time step size (i.e. sampling time) }) \\
\omega_{\text {loop }} & =2 \pi f_{\text {loop }} \\
T F_{\text {loop }, \text {-domain }} & =\frac{1}{1+\frac{s}{\omega_{\text {loop }}}} \\
T F_{\text {loop }, z-\text { domain }} & =c 2 d\left(T F_{\text {loop }, \text { s-domain }}, T_{s},{ }^{\prime} \text { tustin }^{\prime}\right)
\end{aligned}
$$

\subsection{TDC Model}

The coarse TDC has been implemented as an ideal counter as shown in Figure 3.8 The fine TDC model is implemented using an ADC that quantizes abolute phase error. To model the effect of finite resolution, the ADC parameters were set to have 5 bits of quantization levels with a total dynamic range of $250 \mathrm{ps}$ which translates to about 8ps of resolution. However, the model assumes that a simulation discrete step time is orders of magnitude smaller compared to the resolution of the time-to-digital converter. A simulation step size of $100 \mathrm{fs}$ was chosen for this demonstration.

An alternative way to model the effect of finite TDC resolution $\left(T_{\mathrm{LSB}}\right)$, is to compute the equivalent phase deviation at steady-state output frequency caused by the time-to-digital converter. It can then be used to set the value of $k_{D C O}$, which represents the DCO gain in Hz/LSB ( (3.4)), assuming that the loop filter corner frequency dominates the loop update rate [4]. Such a modelling approach restricts its usability only to target a frequency with severely limited tuning range ability, but should be sufficient to spot-check system operation, especially for the purposes of a demonstration. 


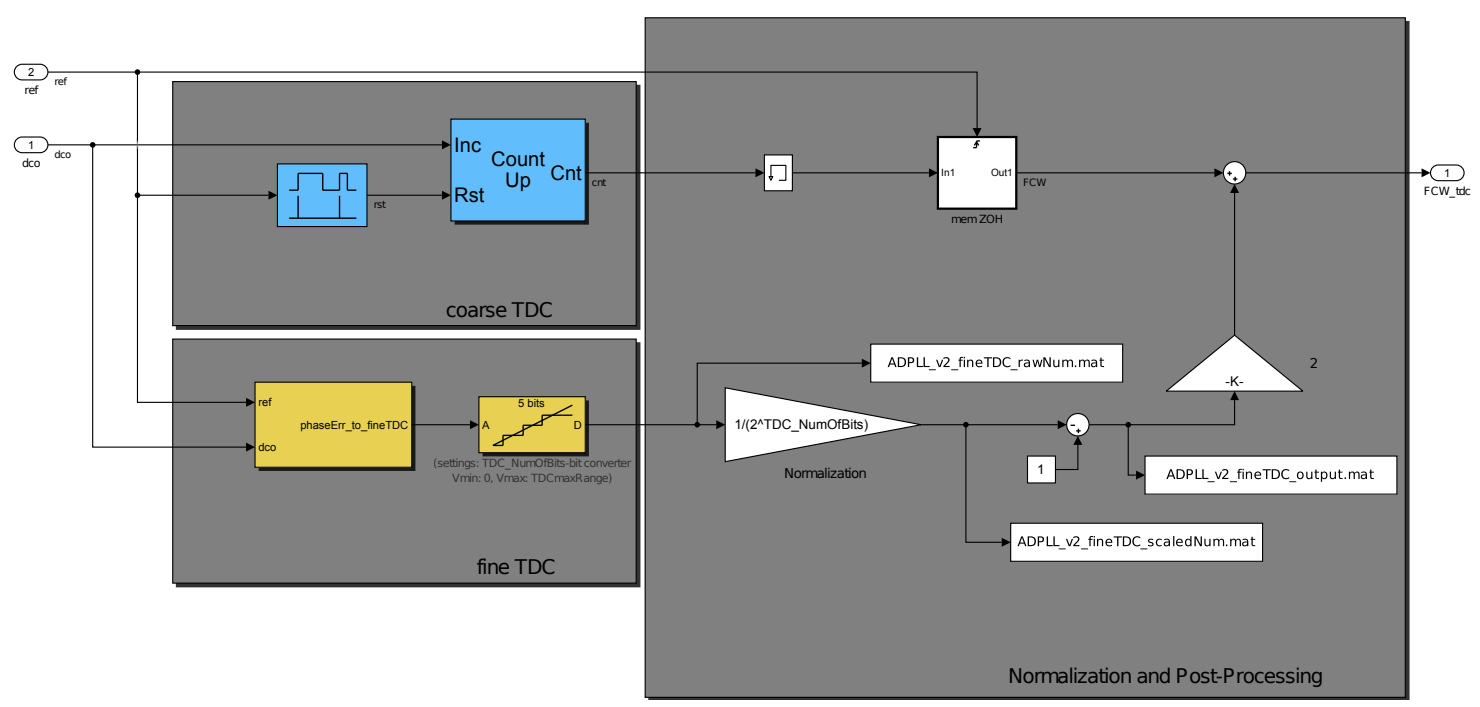

Figure 3.8: TDC model for ADPLL including coarse loop counter and fine phase quantizer

Another alternative approach, including VHDL code, is given in 44.

$$
\begin{aligned}
k_{D C O} & =f_{\text {loop }} \cdot T_{L S B} \cdot f_{D C O}(H z / L S B) \\
& =1 \mathrm{MHz} \cdot 8 \mathrm{ps} \cdot 5 \mathrm{GHz}=40 \mathrm{kHz}
\end{aligned}
$$

\subsection{Behavioural System Model Demonstration}

The result of the simulation is shown in Figure 3.9. which confirms the ability of the ADPLL loop to maintain a $5 \mathrm{GHz}$ output frequency in the presence of DCO noise and quantization of the $\mathrm{TDC}$ due to its finite resolution of $\mathrm{T}_{\mathrm{LSB}}=8 \mathrm{ps}$. 

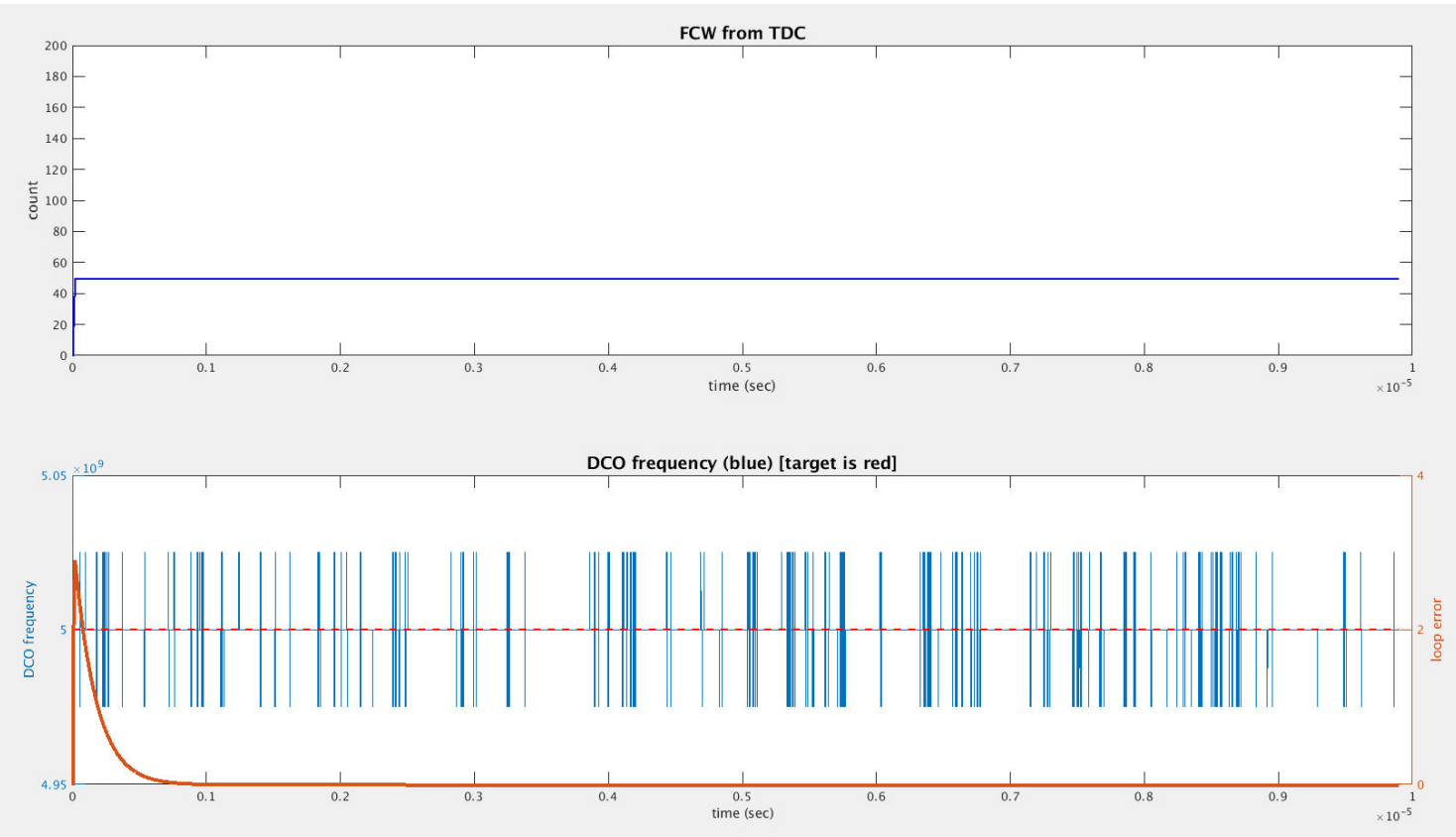

Figure 3.9: A closed-loop simulation of an ADPLL in Simulink with $\mathrm{LBW}=1 \mathrm{MHz}$ $\mathrm{FCW}=50$

\subsection{Summary of the System Model Simulation}

A time-to-digital converter has been modelled and simulated in a discrete-time domain behavioural model of an ADPLL. A digitally-controlled oscillator (DCO) with a realistic and highly accurate phase noise model has also been included in the loop. To control the amount of feedback, a digital filter has been designed to provide some averaging. The closed-loop simulation has demonstrated the ability of an all-digital PLL to successfully synthesize a $5 \mathrm{GHz}$ output clock in the presence of a finite TDC resolution (quantization) and DCO noise. 


\section{Chapter 4}

\section{TDC Performance Metrics and Specifications}

In this chapter TDC performance figures are derived. The relationship between various metrics is established with respect to ADPLL applications. The chapter is concluded with a detailed list of proposed specifications for this work. A custom figure of merit is introduced for comparative purpose with other works.

\subsection{TDC Performance Metrics}

The TDC characterization metrics are similar to those found in a conventional ADC, namely resolution, dynamic range, non-linearity, noise, and effective number of bits (ENOB). However, some of them require further redefinition specific to TDC design. The moment in time when the start signal arrives is labelled as $\mathrm{T}_{\text {start }}$, while the arrival of the stop signal occurs at time $\mathrm{T}_{\text {stop }}$. Therefore, a test time interval that the TDC converts can be expressed by 4.1.

$$
\mathrm{T}_{\text {test }}=\mathrm{T}_{\text {stop }}-\mathrm{T}_{\text {start }}
$$




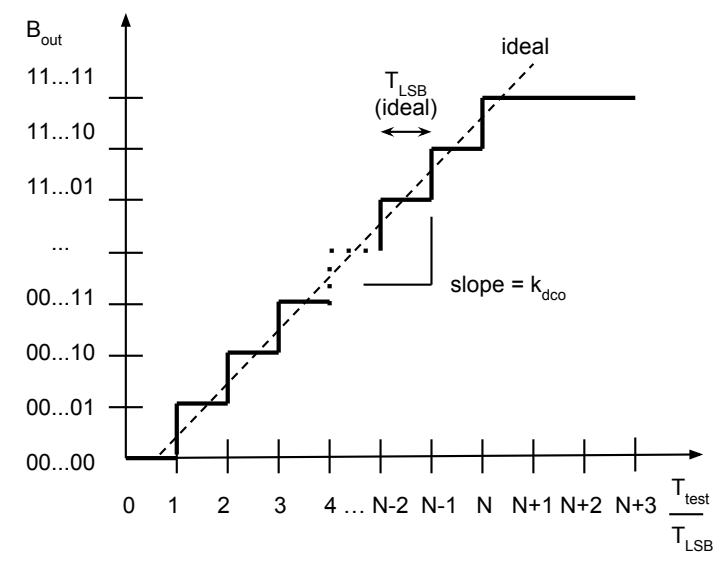

Figure 4.1: Ideal input-output transfer function of TDC.

An ideal TDC transfer function of digital output code $B_{\text {out }}$ versus the input test time interval is shown in Figure 4.1 .

Due to TDC quantization, multiple time intervals $\mathrm{T}_{\text {test }}$ map to the same output code. The minimum width of $\mathrm{T}_{\text {test }}$ that corresponds to a $1 \mathrm{LSB}$ increment of $\mathrm{B}_{\text {out }}$ is the absolute resolution $\mathrm{T}_{\mathrm{LSB}}$. Mathematically this relationship can be expressed by eqation 4.2 .

$$
T_{\text {test }}=B_{\text {out }} T_{L S B}+\epsilon \quad 0 \leq \epsilon \leq T_{L S}
$$

The $\epsilon$ represents quantization error. The ideal dynamic range of a TDC can be expressed by 4.3 .

$$
D R=2^{N} \cdot T_{L S B}
$$

However, non-idealities such as gain, offset, noise, and non-linearity will degrade the effective resolution and dynamic range. 


\subsubsection{TDC Quantization Error}

Assuming uniform error distribution, the mean quantization error $\epsilon$ is defined by (4.4).

$$
\langle\epsilon\rangle=\frac{1}{T_{L S B}} \int_{i=0}^{T_{L S B}} \epsilon d \epsilon=\frac{1}{2} T_{L S B}
$$

The quantization noise power is then 4.5 [3].

$$
\left\langle\epsilon^{2}\right\rangle=\frac{1}{T_{L S B}} \int_{i=0}^{T_{L S B}} \epsilon^{2} d \epsilon=\frac{1}{3} T_{L S B}^{2}
$$

The total error is the result of a constant $\langle\epsilon\rangle$ and a mean free $\langle\eta\rangle$ component (4.6).

$$
\begin{aligned}
\left\langle\epsilon^{2}\right\rangle & =\langle\epsilon\rangle^{2}+\left\langle\eta^{2}\right\rangle \\
& =\frac{1}{4} T_{L S B}^{2}+\frac{1}{12} T_{L S B}^{2}
\end{aligned}
$$

For an n-bit TDC the quantization SNR can be estimated by 4.7

$$
S N R=6.02 \cdot n+1.76 d B
$$

Just as in ADC converters, each additional bit of TDC resolution improves SNR by $\approx 6 d B$.

\subsubsection{Linear Imperfections of a TDC}

A real TDC transfer function can deviate from the ideal as shown in Figure 4.2 Drawing a line of best fit (or end-to-end) helps understand and analyze TDC error contributors. The linear imperfections, discussed in this section, are caused by offset 


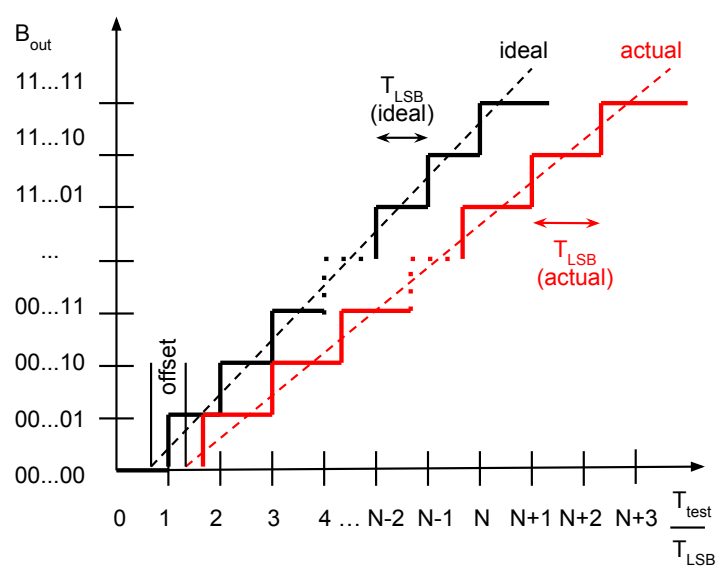

Figure 4.2: Linear imperfections causing changes in input-output transfer function characteristic of a TDC.

(additive) and slope (gain) errors of the linearized transfer fucntion.

A systematic offset error is typically caused by unaccounted phase skew between the start and stop signals. For simplicity, it can be defined as the difference in time between an ideal $\mathrm{T}_{\mathrm{LSB} \text {,ideal }}$ and an actual $\mathrm{T}_{\mathrm{LSB} \text {,actual }}$ to transition from $\mathrm{B}_{\text {out }}=0$ by 1LSB. The time when the TDC output code observes the first transition is denoted as $\mathrm{T}_{00 . .01}$. Mathematically the offset error can be expressed by 4.8

$$
E_{o f f s e t}=\frac{T_{00 \ldots 01}-T_{L S B}}{T_{L S B}}
$$

Evidently, the offset error can be linearly subtracted, thus allowing a linear gain error which might be caused by PVT variations or deviation from desired TDC bias conditions. The error can be calculated from the slope of the linearized transfer function, $\mathrm{k}_{\mathrm{TDC}} \quad 4.9$.

$$
k_{T D C}=\frac{\Delta B_{\text {out }}}{\Delta T_{\text {test }}}
$$

For an ideal transfer function 4.9 reduces to 4.10 , which implies 1 LSB change 
for a time interval $T_{L S B, \text { ideal }}$.

$$
k_{T D C}=\frac{1}{\Delta T_{L S B}}
$$

The deviation of actual slope from ideal can be attribted to gain error in 4.11.

$$
E_{\text {gain }}=\frac{T_{11 \ldots 11}-T_{00 \ldots 01}}{T_{L S B \text { ideal }}}-\left(2^{N}-2\right)
$$

A more general form of the TDC transfer function slope can be re-written as (4.12).

$$
k_{T D C}=k_{T D C \text { ideal }}\left(1-\frac{E_{\text {gain }}}{2^{N}-2}\right)
$$

If there is no gain error, then the equation reduces back to the ideal form of 4.10.

Such impairments as offset and gain can usually be compensated for using a forward calibration routine or a form of closed feedback trimming. Once the linear sources of error are removed, the transfer function can be re-evaluated to reveal other sources of errors such as non-linear behaviour discussed in Section 4.1.3

\subsubsection{Non-linear characteristics of a TDC}

The TDC transfer function may also deviate from ideal characteristic in non-linear fashion. This might be due to inherent non-uniformity of the TDC by design or due to poor implementation of various elements causing unequal delays, mismatches, or metastability within the time-to-digital converter core circuitry. Analogous to ADC nomenclature, two main categories can be identified: integral and differential nonlinearity.

The Integral Non-Linearity (INL) is a measure of macroscopic deviations of the TDC transfer function across its entire dynamic range. The ideal TDC input-output 


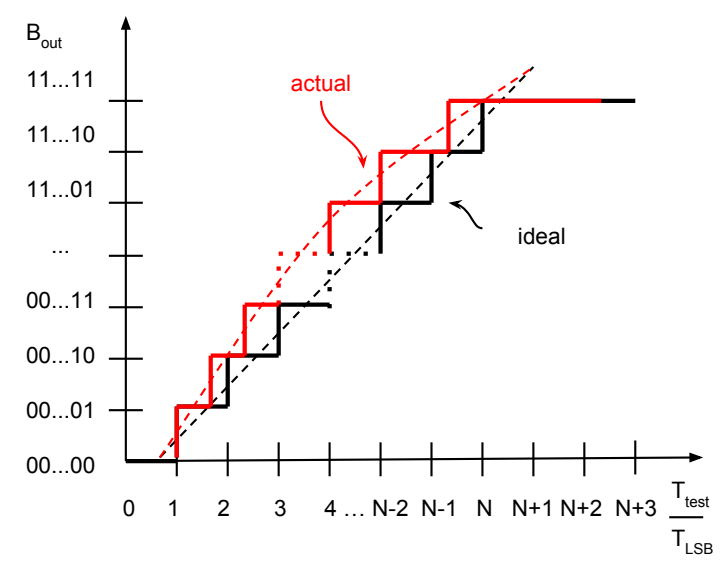

Figure 4.3: Integral non-linear imperfections causing changes in input-output transfer function characteristic of TDC.

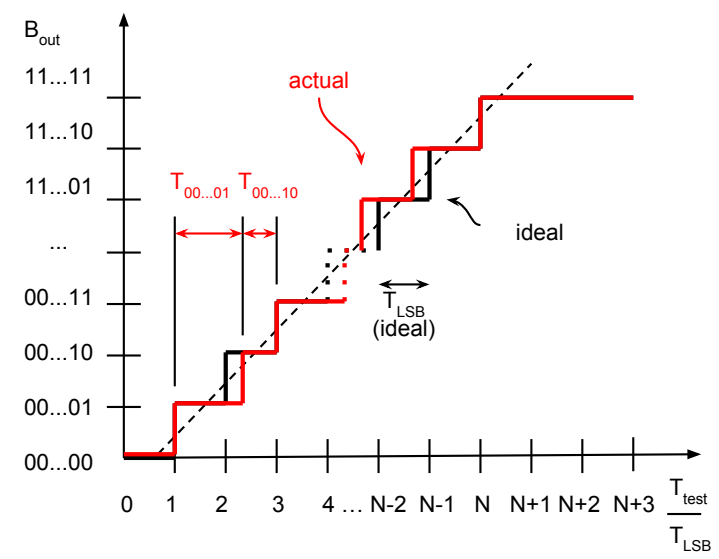

Figure 4.4: Differential non-linear imperfections causing changes in input-output transfer function characteristic of TDC.

behaviour can be approximated using the best fit line method, after removing linear offset and gain errors discussed in Section 4.1.2 An example showing the effect of integral non-linearity is shown in Figure 4.3

The differences between converter actual step sizes at each level from ideal $\mathrm{T}_{\mathrm{LSB} \text {,ideal }}$ are represented by Differential Non-Linearity (DNL). An example of a converter affected by the DNL error is shown in Figure 4.4 . 


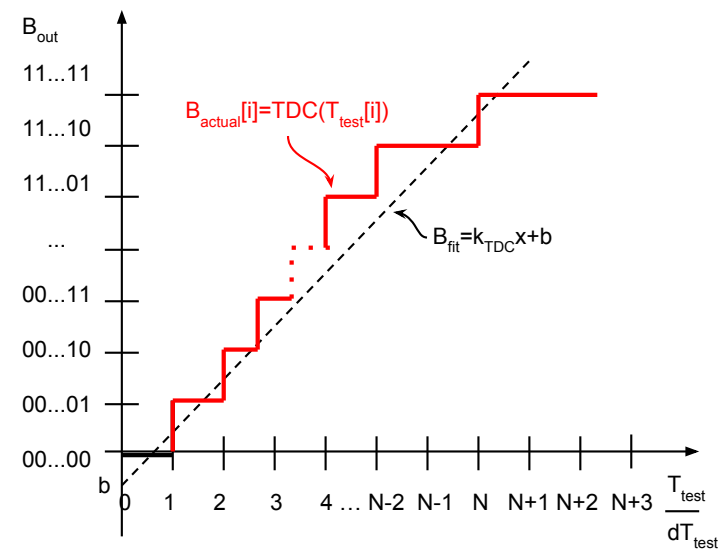

Figure 4.5: Computational principle of INL.

Qualitatively it can be seen that large DNL excursions may cause some output codes $\mathrm{B}_{\text {out }}$ to be completely missing. In an ADPLL this might cause rise to undesired system noise. For a well-behaved and low noise TDC design, the INL for any output code [i] within the TDC dynamic range, is simply an accumulation (integration) of all DNL up that point, hence, the name integral 4.13.

$$
I N L=\sum_{i=0}^{N} D N L[i]
$$

However, this work adopts a more general way to compute INL, independent of DNL. Mathematically the INL can be expressed as the deviation of the actual output code from the ideal that is determined by a line of best fit for all data points (assuming offset and gain errors are removed) as seen in Figure 4.5 A line of best fit is a linear equation 4.14.

$$
\begin{array}{r}
y=m \cdot x+b \quad \Leftrightarrow \quad B_{o u t, f i t}=k_{t d c, f i t} \cdot T_{\text {test }}+b \\
k_{t d c, f i t}=\frac{1}{T_{L S B}}
\end{array}
$$


Therefore, for each test interval $\mathrm{T}_{\text {test }}[\mathrm{i}]$ and resulting output code $\mathrm{B}_{\text {out }}[\mathrm{i}]$ the INL[i] (normalized to an ideal $\mathrm{T}_{\mathrm{LSB}}$ ) can be computed using 4.15).

$$
\begin{aligned}
I N L[i] & =\frac{B_{\text {out }, \text { actual }}[i]-B_{\text {out }, \text { fit }}[i]}{B_{\text {out }, f i t}[i]} \\
& =\frac{B_{\text {out }, \text { actual }}[i]}{B_{\text {out }, \text { fit }}[i]}-1 \\
& =\frac{B_{\text {out }, \text { actual }}[i]}{k_{\text {tdc,fit }} T_{\text {test }}[i]+b}-1
\end{aligned}
$$

Similar to the ADC nomenclature, only the maximum value $\max |I N L[i]|$ is reported in the design summary.

A commonly used technique called "code density test" can be used to compute DNL [13]. A total of $\mathrm{N}$ test intervals of width $\Delta T_{\text {test }}=\left(T_{\text {test }}[i+1]-T_{\text {test }}[i]\right)$ are applied at the TDC input, uniformly covering its entire dynamic range DR 4.3. Under an assumption that $\Delta T_{\text {test }}<T_{L S B}$, there are going to be multiple $T_{\text {test }}$ [i] that will be quantized by the $\mathrm{n}$-bit TDC to the same output code $\mathrm{B}_{\text {out }}$. Let $\mathrm{k}_{\exp }$ represent the number of $\Delta T_{\text {test }}$ that are expected to land within a $1 \mathrm{LSB}$ of $\mathrm{B}_{\text {out }}$ code 4.16).

$$
k_{\text {exp }}=\frac{N}{2^{n}}
$$

However, the non-ideal nature of the TDC and noise will cause some test intervals to land in adjacent code bins, mainly affecting those test points landing close to code bin edges (transition between adjacent codes). The DNL by code density test can be defined as the error between number of actual $k_{\text {act }}$ versus expected code hits $k_{\exp }$ as shown in 4.17).

$$
D N L[i]=\frac{k_{a c t}-k_{e x p}}{k_{\text {exp }}}
$$

The accuracy of such a DNL definition is limited to $\Delta \mathrm{T}_{\text {test }}$ step size, which ideally 
should be

$$
\Delta T_{\text {test }} \gg \epsilon
$$

such that quantization error $\epsilon$ dominates the noise. Some literature suggests using

$$
\Delta T_{\text {test }} \geq \frac{T_{L S B}}{10}
$$

as a rule of thumb [14] for measurements.

\subsubsection{Single-Shot Precision}

One of the fundamental dynamic performance metrics of a time-to-digital converter is the Single Shot Precision (SSP). It is defined as a standard deviation of output code for a repeatedly applied constant input tests time interval $T_{\text {test }}$ (4.1). It is a TDC alternative metric to signal-to-noise ratio of ADC converters. In traditional ADC characterization, a single-tone measurement is typically performed, during which a continuous sinusoidal sequence of input voltages is applied. The ADC output code is then passed through an ideal digital-to-analog converter (DAC) to reconstruct the analog signal (or the output code of ADC is low-pass filtered) [2]. Using frequency decomposition technique, such as a discrete fourier transform (DFT), it is feasible to measure the signal power at a fundamental test tone frequency $\mathrm{P}_{\text {signal }}$, its harmonics $\left(\mathrm{P}_{\text {harmonics }}\right)$, and noise floor power $\left(\mathrm{P}_{\text {noise }}\right)$. The signal-to-noise $(\mathrm{SNR})$ and signal-to-noise-and-distortion (SNDR) ratio can then be computed in (4.18) and 4.19 respectively.

$$
\begin{gathered}
S N R=10 \cdot \log _{10}\left(\frac{P_{\text {signal }}}{P_{\text {noise }}}\right) \\
S N D R=10 \cdot \log _{10}\left(\frac{P_{\text {signal }}}{P_{\text {noise }}+P_{\text {harmonics }}}\right)
\end{gathered}
$$


The dynamic SNR includes the effects of quantization and other types of noises, while SNDR also includes the linearity impairments, which degrades with larger input amplitude (peak-to-peak sinusoidal input). For very low input amplitudes, below the resolution of the ADC, the SNR is very low. Conversely, with increased input amplitude the SNR continues to grow up to a point $\mathrm{SNR}_{\max }$ where the ADC output starts to saturate, which leads to subsequent degradation of SNR beyond this point. The effective dynamic range of an $\mathrm{ADC}$ can be considered the range of input amplitudes which satisfies all $\mathrm{SNR}$ values within the range $0<\mathrm{SNR}<\mathrm{SNR}_{\max }$.

Applying similar methodology to TDC is more difficult due to the complexity of constructing a continuous sinusoidal sequence of time periods with resolution exceeding that of the TDC itself [15]. However, by observing the standard deviation $\sigma$ of the TDC output code for a given test period, it is possible to measure the noise contribution of the TDC. Test time intervals of different duration can be applied to an TDC to measure the SSP across the entire dynamic range of the converter. To better understand the dominant contributors to SSP, a double-shot precision test can be applied. Two test time intervals are repeatedly converted by a TDC and two SSP series are extracted. Using auto and cross-correlation functions it is then possible to deduce which part of the TDC circuit contributes most of the noise [3. The uncertainty of the TDC output due to noise degrades the instantaneous accuracy of the converter. Averaging the TDC output can help reduce the error. Knowing SNR (ADC) and SSP (TDC) figures may help system designers determine the amount of averaging required for a specific application.

\subsubsection{Effective Number of Bits}

Another way of looking at TDC dynamic performance instead of SNR figures is to convert it back into resolution. As described in Section 4.1.1 the n-bit converter has a 
fundamental limit to attainable SNR 4.7). The actual SNR measurement will most likely be worse due to additional noise and linearity impairments. The alternative way of looking at SNR degradation is to find the effective number of bits (ENOB) that would be required for another ideal converter to achieve the level of SNR degraded by the noise. Instead of SNR, the TDC metric describing measurement uncertainty is the single-shot precision discussed in Section 4.1.4 The ENOB and effective $T_{L S B}^{*}$ resolution can be computed using 4.20 and 4.21 respectively.

$$
E N O B=\log _{2}\left(\frac{D R}{\sqrt{12} \cdot S S P}\right)
$$

Subsequently, the fewer effective number of bits for a given dynamic range can be seen as though TDC resolution $\mathrm{T}_{\mathrm{LSB}}$ is increased [3]. A simplified effective resolution can be given as 4.21

$$
\begin{aligned}
T_{L S B}^{*} & \approx \frac{D R}{2^{E} N O B}=\frac{2^{n} \cdot T_{L S B}}{2^{E} N O B} \\
& =2^{n-E N O B} \cdot T_{L S B, \text { ideal }}
\end{aligned}
$$

The calculations above assume normal distribution of TDC quantization noise. In practice, the noise would likely be larger, so additional margin should be built in for application. 


\subsection{Figure of Merit}

A figure of merit helps compare various designs against each other by normalizing performance to power, area, and frequency of operation. There are two commonlyaccepted figures of merit in the TDC reference literature:

$$
\begin{aligned}
& F O M_{P}=\frac{\text { Power }}{f_{\text {ref }} \cdot 2^{E N O B}} \\
& F O M_{A}=\frac{\text { Area }}{f_{\text {ref }} \cdot 2^{E N O B}}
\end{aligned}
$$

However, these figures of merit assume that both start and stop signals arrive at an equal rate of $\mathrm{f}_{\mathrm{ref}}$. For some architectures, including this work, such a definition would be inaccurate as a significant part of the circuitry may operate at a much higher rate, thus increasing the dynamic power consumption. Moreover, some works achieve much better raw FOM 4.22 by moving to newer technology nodes. To compare architectures, it would be informative to decouple technology advantages from implementation techniques, since practically all designs can be ported to smaller technology nodes and benefit from lower power consumption. Lastly, the cost of manufacturing is heavily influenced by area consumption and lithography (technology).

Therefore, a cost-sensitive (and partially technology-independent) figure of merit is proposed in 4.24.

$$
F O M_{P A C}^{*}=\frac{\sum_{i=1}^{N} \frac{\text { Power }_{i}}{f_{i}} \cdot \text { Area }_{m m^{2}} \cdot \text { Cost }_{\$ / m m^{2}}}{2^{E N O B}} \quad[\$ \cdot \text { Joules } / \text { bit }]
$$

The Power ${ }_{i}$ and $f_{i}$ refer to dynamic power normalized to the system frequency component that contributes to it. The latest information of cost was obtained from 
CMC Microsystems [1] and summarized in table Table 4.1

Table 4.1: Cost of manufacturing - academic pricing [1].

\begin{tabular}{lc}
\hline Prototype Manufacturing Technology $\quad$ through CMC & Prices $\$ / \mathrm{mm}^{2}$ (Canadian Academic Price) \\
\hline STMicroelectronics $28 \mathrm{~nm}$ CMOS FD SOI (through CMP) & 12000 \\
TSMC $65 \mathrm{~nm}$ CMOS GP (through MOSIS) & 6100 \\
TSMC 0.13 um CMOS (through MOSIS): CR013G & 2500 \\
TSMC 0.18 um CMOS (through MOSIS): CMOSP18 & 1350 \\
TSMC $0.35 \mu \mathrm{m}$ CMOS (through MOSIS): CMOSP35 & 475 \\
\hline
\end{tabular}

\subsection{Deriving the Target Specifications}

The specification of LPI-TDC are derived for an ADPLL application. Given the highly integrated nature of the ADPLL system, an upper target frequency $5 \mathrm{GHz}$ was selected (e.g. WLAN 802.11), which allows for an easy on-chip integrated antenna. The re-configurable nature of the ADPLL should allow for a wide range of frequencies down to GSM [16] bands of about $1 \mathrm{GHz}$, thus also covering such applications as Bluetooth at $2.4 \mathrm{GHz}$. The frequency tuning range translates into a phase quantizer Dynamic Range requirement of 200 ps - 1 ns. As covered earlier in Section 2.1.2. the phase quantizer may consist of a coarse counter and fine TDC components. For demonstration purposes, it was decided to keep the fine TDC to 5 bits, while the rest of dynamic range can be covered by a coarse counter or a loop configuration (see Section 2.5). A quick search of recent publications has yielded several examples of high-speed counters which were implemented and measured in a similar generation of technology as this work and target frequency rates $17-19$.

The LPI-TDC resolution translates to quantization noise which limits the ADPLL 
performance [20]. The single-side band phase noise spectrum is defined by 4.25

$$
S_{d B}=10 \log _{10}\left(\frac{\left(2 \pi f_{D C O} \cdot \sigma_{t}\right)^{2}}{f_{\text {ref }}}\right)
$$

Assuming the TDC timing uncertainty $\sigma_{t}$ is dominated by white quantization noise, (4.6) can be substituted into 4.25 to relate the resolution and effective phase noise spectrum 4.26.

$$
S_{d B}=10 \log _{10}\left(\frac{\left(2 \pi f_{D C O} \cdot T_{L S B}\right)^{2}}{12 \cdot f_{\text {ref }}}\right)
$$

A Banerjee's figure of merit 4.27) for a normalized noise floor of $-215 \mathrm{~dB}$ was selected to satisfy most GSM application requirements and leave $10 \mathrm{~dB}$ margin (over $205 \mathrm{~dB}$ worst case), thus making it sufficient to cover a broad range of RF applications [4.21].

$$
\begin{aligned}
B F M_{d B} & =S_{d B}-10 \log _{10} f_{r e f}-20 \log _{10}\left(\frac{f_{D C O}}{f_{r e f}}\right) \\
& =10 \log _{10}\left(\left(2 \pi f_{D C O} \cdot \sigma_{t}\right)^{2}\right) \\
& =10 \log _{10}\left(\frac{\left(2 \pi f_{D C O} \cdot T_{L S B}\right)^{2}}{12}\right)
\end{aligned}
$$

The equation (4.27) can now also be used to estimate the single-shot precision margin and the effective number of bits as derived in Section 4.1.5

\subsection{Summary of LPI-TDC Specifications}

A discussion and derivation of performance metrics applicable to a time-to-digital converter have been presented in Section 4.1 A cost-sensitive Figure of Merit (FOM) has been introduced to enable a comparison between various architectures across a wide range of technologies. An analytical relationship has then been established 
between the TDC parameters and a typical application requirements. A comprehensive specification summary along with a few application scenarios are presented in Table 4.2 .

Table 4.2: List if LPI-TDC specifications for ADPLL application. Several scenarios presented to show the effect of design margin.

\begin{tabular}{|c|c|c|c|c|c|}
\hline Parameter & units & $\begin{array}{l}5 \mathrm{GHz} \\
\text { Case } 1\end{array}$ & $\begin{array}{l}5 \mathrm{GHz} \\
\text { Case } 2\end{array}$ & $\begin{array}{l}5 \mathrm{GHz} \\
\text { Case } 3\end{array}$ & $\begin{array}{l}1 \mathrm{GHz} \\
\text { Case } 1\end{array}$ \\
\hline $\mathrm{BFM}_{\mathrm{dB}}$ & $\mathrm{dB}$ & -215 & -210 & -205 & -215 \\
\hline ENOB & LSB & 4.7 & 4 & 3 & 4.7 \\
\hline $\mathrm{T}_{\mathrm{LSB}, \mathrm{eff}}$ & ps & 9.80 & 1.74 & 3.10 & 9.80 \\
\hline n (bits) & LSB & 5 & 5 & 5 & 5 \\
\hline $\mathrm{f}_{\mathrm{DCO}}$ & $\mathrm{Hz}$ & $5.0 \mathrm{G}$ & $5.0 \mathrm{G}$ & $5.0 \mathrm{G}$ & $1.0 \mathrm{G}$ \\
\hline $\mathrm{f}_{\mathrm{ref}}$ & $\mathrm{Hz}$ & $100 \mathrm{M}$ & $100 \mathrm{M}$ & $100 \mathrm{M}$ & $100 \mathrm{M}$ \\
\hline $\mathrm{S}_{\mathrm{ssb}}$ & $\mathrm{dBc} / \mathrm{Hz}$ & -101 & -96 & -91 & -115 \\
\hline Dynamic Range (fine TDC core) & ps & 255 & 279 & 248 & 255 \\
\hline $\mathrm{T}_{\mathrm{LSB}, \text { design }}$ & ps & 7.96 & 8.72 & 7.75 & 7.96 \\
\hline $\mathrm{SSP}_{\mathrm{ps}}$ & ps & 2.83 & 5.03 & 8.95 & 2.83 \\
\hline $\mathrm{SSP}_{\mathrm{LSB}}$ & LSB & 0.36 & 0.58 & 1.15 & 0.36 \\
\hline VDD & $\mathrm{V}$ & 1.40 & 1.40 & 1.40 & 1.40 \\
\hline $\begin{array}{l}\text { Interpolator Power } \\
\left.\text { (at } \mathrm{f}_{\mathrm{DCO}}\right)\end{array}$ & $\mathrm{mW}$ & 94.50 & 94.50 & 94.50 & 14.00 \\
\hline $\begin{array}{l}\text { IO Buffers } \\
\left(\text { at } f_{\text {ref }}\right)\end{array}$ & $\mathrm{mW}$ & 10.50 & 10.50 & 10.50 & 8.40 \\
\hline $\begin{array}{l}\text { Total Power Consumtion } \\
\text { (fine TDC core) }\end{array}$ & $\mathrm{mW}$ & 105.00 & 105.00 & 105.00 & 22.40 \\
\hline Area & $\mathrm{mm}^{2}$ & 0.20 & 0.20 & 0.20 & 0.20 \\
\hline $\begin{array}{l}\mathrm{FOM}_{\mathrm{P}} \\
\text { (fine TDC core) }\end{array}$ & Joules/bit & $4.77 \mathrm{p}$ & $7.74 \mathrm{p}$ & $1.55 \mathrm{p}$ & $3.77 \mathrm{p}$ \\
\hline Counter Specification & count & 40 & 40 & 40 & 40 \\
\hline $\begin{array}{l}\text { Power Consumption } \\
\text { (coarse counter estimate [ [17]]) }\end{array}$ & $\mathrm{mW}$ & 5 & 5 & 5 & 5 \\
\hline Dynamic Range with counter & ps & $10.2 \mathrm{E}+3$ & $11.2 \mathrm{E}+3$ & $9.9 \mathrm{E}+3$ & $10.2 \mathrm{E}+3$ \\
\hline $\begin{array}{l}\mathrm{FOM}_{\mathrm{P}} \\
\text { (coarse counter \& fine TDC core) }\end{array}$ & Joules/bit & $120.1 \mathrm{f}$ & $195.2 \mathrm{f}$ & $390.3 \mathrm{f}$ & $99.1 \mathrm{f}$ \\
\hline $\begin{array}{l}\text { FOM }_{\text {PAC }} \\
\text { (coarse counter [ [17] }] \& \text { fine TDC core) }\end{array}$ & $\$$.Joules/bit & $48.1 \mathrm{p}$ & $78.1 \mathrm{p}$ & $156.1 \mathrm{p}$ & $39.6 \mathrm{p}$ \\
\hline
\end{tabular}




\section{Chapter 5}

\section{Design of LPI-TDC}

This chapter presents implementation steps for a 5-bit time-to-digital converter based on the local passive interpolation. Schematic design and simulations were done using Cadence tools release 5.1 and later ported into a newer version 6.1, which enabled the use of the improved simulation tools and analyses. All simulations were done using Cadence Spectre engine and the IBM 0.13 $\mu \mathrm{m}$ CMOS BSIM4v4 MOSFET models (v1.8.0.4DM) from the Process Design Kit (PDK) provided by the foundry. To simplify the analysis, the architecture was partitioned into individual building blocks: interpolation delay cell (Section 5.1), Flip-Flop (latch) (Section 5.3), and input/output (I/O) buffers (Section 5.4 capable of driving large capacitive loads. The top-level diagram of the proposed LPI-TDC is shown in Figure 5.1. The discussion is accom-

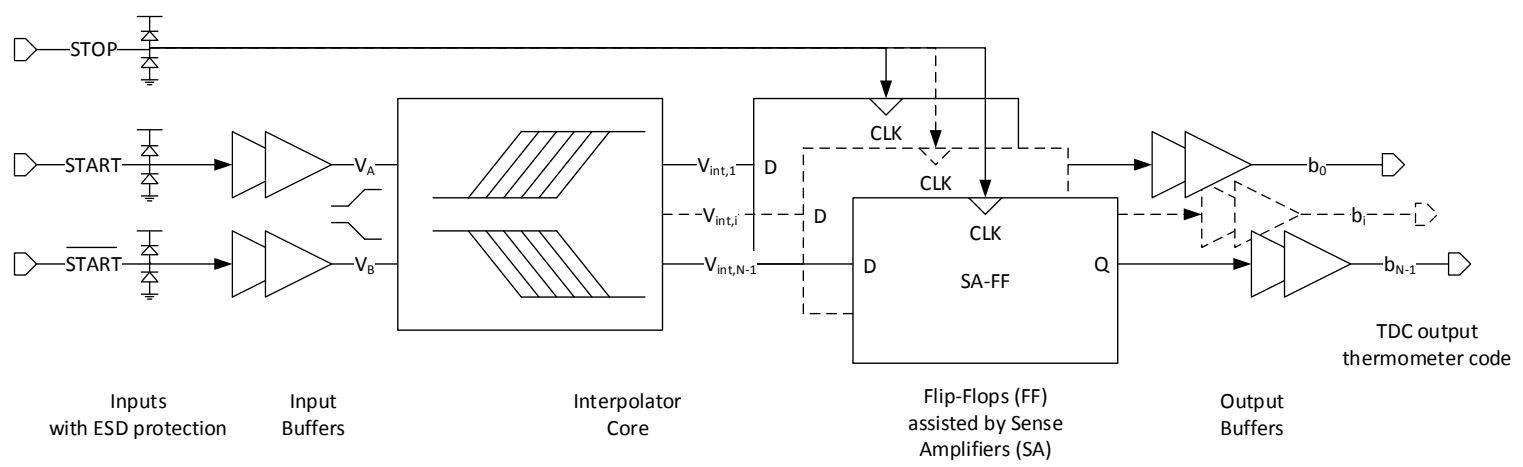

Figure 5.1: Block level diagram of the proposed LPI-TDC circuit. 
panied with layout considerations, implementation, while the chip-level floorplan and integration are presented in Section 5.5

\subsection{Interpolation Delay Cell}

The interpolation core shown in Figure 5.1 consists of multiple delay cells arranged in series. Each cell includes a delay element and local interpolator (voltage divider). As discussed in Section 2.4.3, each delay element should take an input signal $\mathrm{V}_{\mathrm{A}}$ to generate an output signal $V_{B}$ delayed by a single $t_{d}^{\text {gate }}$ (inverter propagation delay). A differential digital delay element is used to achieve this task. The two inverters

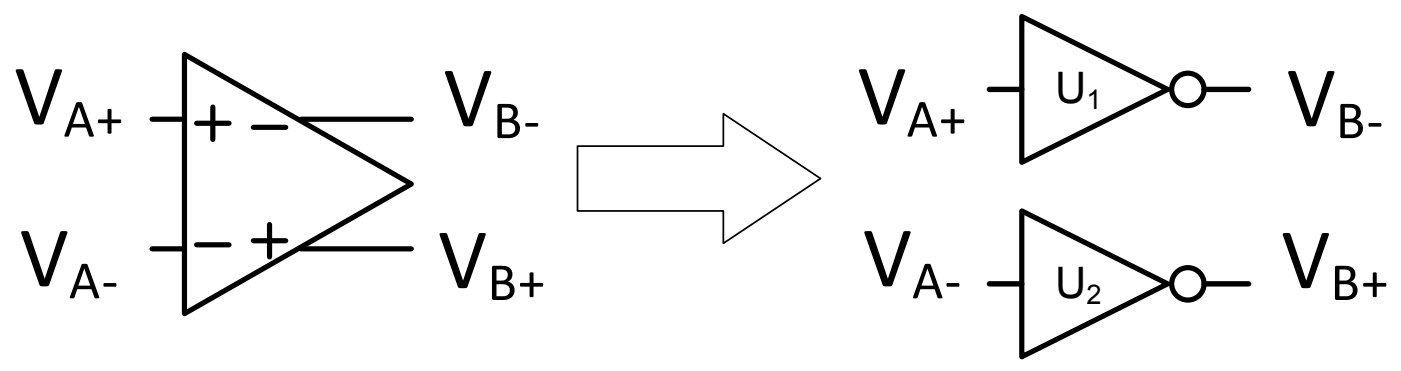

Figure 5.2: TDC differential delay element.

$\left(\mathrm{U}_{1}\right.$ and $\left.\mathrm{U}_{2}\right)$ are designed to have identical drive strength, thus providing equal delay $t_{d}^{\text {gate }}$ with opposite polarities. To perform interpolation, the input and output of each cell is connected to a voltage divider in cross-coupled fashion as shown in Figure 5.3

The interpolation factor IF and circuit device sizes are now determined to satisfy the desired resolution $\mathrm{T}_{\mathrm{LSB}} \approx 8 \mathrm{ps}$. Therefore, $\mathrm{t}_{\mathrm{d}}^{\text {gate }}$ and IF parameters have to be defined. The propagation delay $\mathrm{t}_{\mathrm{d}}^{\text {gate }}$ is mainly a function of inverter device sizing, while the IF is determined by number of interpolation elements.

The analysis begins with understanding the passive voltage divider limitations. 


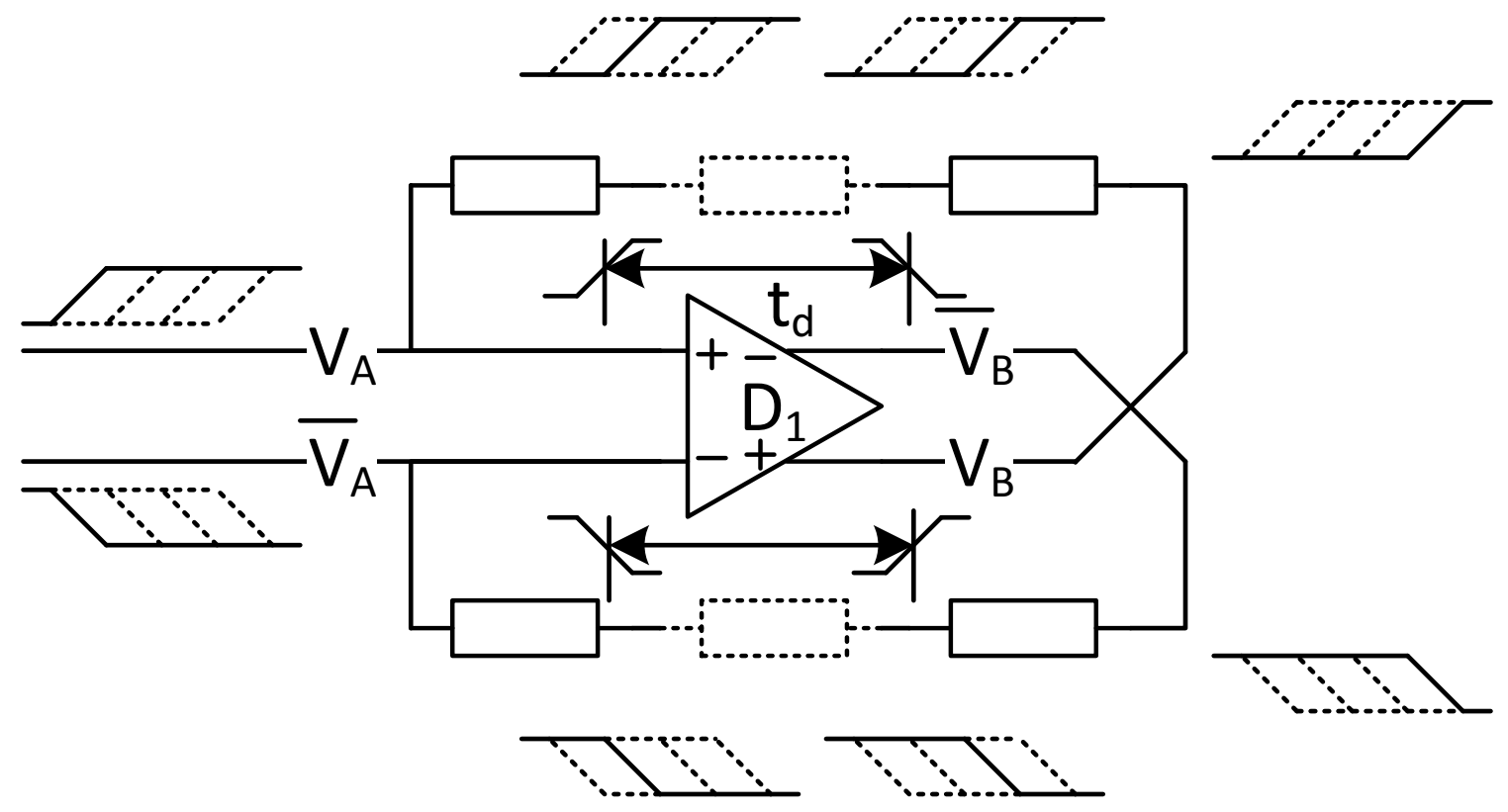

Figure 5.3: TDC delay cell interpolation principle.

To minimize variation of interpolation action, maintain good linearity and low capacitance across various PVT conditions, it was decided to use passive resistors, as opposed to active elements such as transistor-based switches or diodes. The thermal noise of the resistor is expected to be negligible compared to the signal slopes arriving at comparator inputs.

Based on IBM design documentation for cmrf8sf process flavour, the resistor choices have been narrowed down to $\mathrm{P}+$ Polysilicon (opppcres) and RP Polysilicon (oprppres) models. The interpolation by voltage division implies that the TDC resolution should not strongly depend on absolute value of resistors. The main requirement is that they match well enough within a delay cell. However, it is still desirable that the absolute resistance value does not significantly increase due to PVT variation to avoid large time constants that could compromise the speed of interpolation (product of resistor and parasitic capacitances). The $\mathrm{P}+$ Polysilicon resistor 
was ultimately selected due to significantly better area efficiency over RP Polysilicon, benefitting from higher sheet resistivity $\left(\rho_{\text {pppcres }}>\rho_{\text {rppres }}\left(\frac{\Omega}{s q r}\right)\right)$. The opppcres resistor is also second best in this technology for PVT variation due to marginally larger temperature coefficient (tempco) compared to oprppres. This variation due to tempco can be mitigated through other design trade-offs if needed (e.g. power). The absolute percent error of opppcres for a range of resistance values and temperatures is plotted in Figure 5.4 .

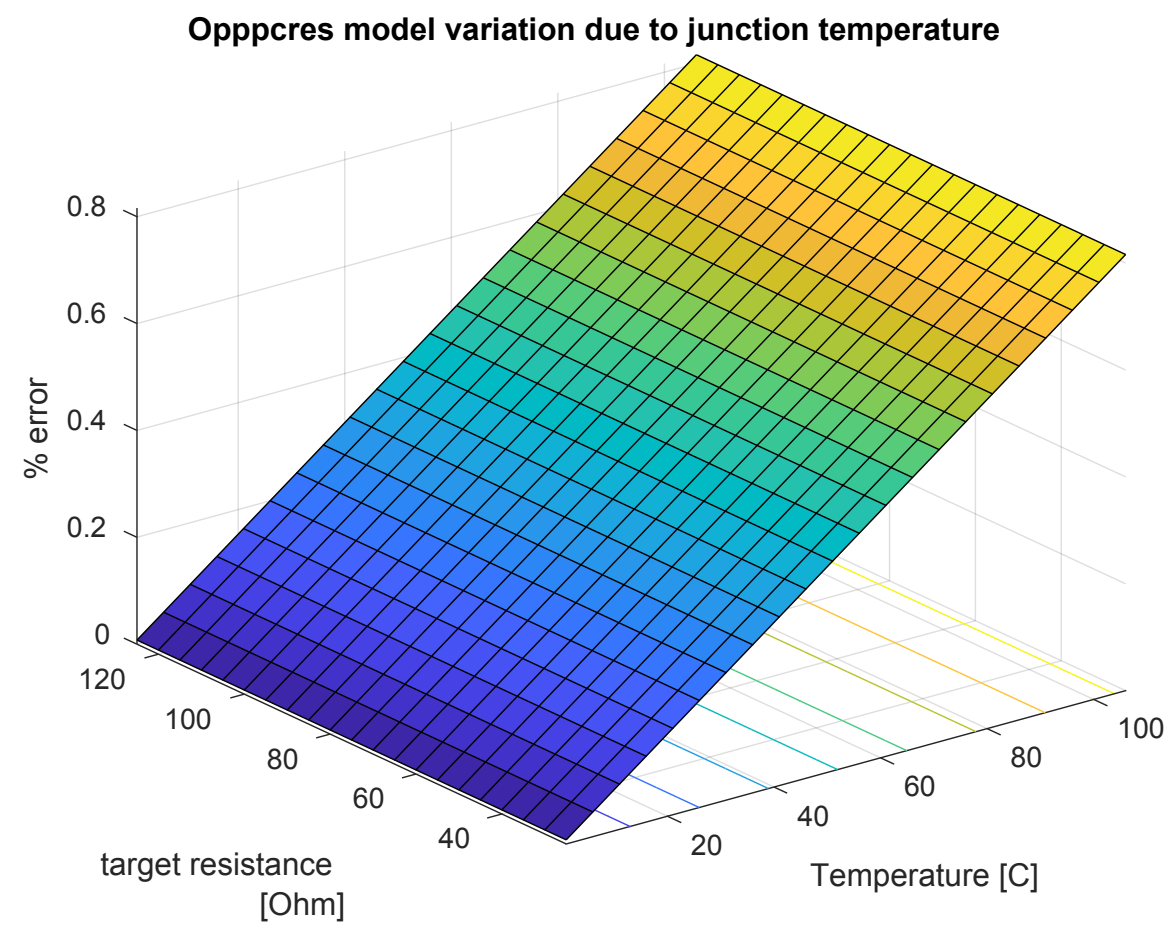

Figure 5.4: Error of final resistance value of opppcres model compared to rppres for a range of target resistance values and temperature variation.

The upper limit of resistor value is determined by maximum $\mathrm{RC}$ time constant that can be tolerated, while the minimum value is constrained by power and area. Based on preliminary layout estimates, the interconnect parasitics were assumed to be on the order of $10 \mathrm{fF}$ due to metal-to-metal and metal-to-substrate capacitances. 
This comes in addition to parasitic capacitance due to downstream circuit load, such as the Sense-Amplifier assisted Flip-Flop (SAFF) as depicted in Figure 5.1 A total of $100 \mathrm{fF}$ is budgeted for parasitic capacitance at interpolation node. The start signal target frequency is $5 \mathrm{GHz}$, which has a period of 200 ps. For robust operation at such frequencies, signal edges should have rise time on the order of $t_{\text {rise }}=\frac{1}{5} T_{\text {period }}=40 \mathrm{ps}$. The RC time constant of interpolator should be faster than that, to guarantee linear interpolation at such frequencies, so an additional factor 4 is added to the margin, making it a total of $\frac{1}{20}$ at interpolation nodes. Using the formula from $[2$, the $\mathrm{RC}$ product is calculated in (5.1).

$$
t_{\text {rise }} \approx 2.2 \cdot \tau_{R C}=2.2 \cdot R \cdot C
$$

Rearranging (5.1) and substituting known values shows the required target resistance at each node to be apprximately $50 \Omega(5.2)$.

$$
R=\frac{20 p s}{2.2 \cdot 100 f F}=45.5 \Omega
$$

This value is very well suited to be implemented using $\mathrm{P}+$ Polysilicon resistor while occupying small layout footprint and low parasitic self-capacitance.

The next step is to design an inverter with sufficiently low propagation delay. Assuming that start signal rise time is $40 \mathrm{ps}$, as estimated earlier, the gate delay time requirement can be approximated by 3.3 .

$$
t_{d}^{\text {gate }}=\frac{1}{2} t_{\text {rise }} \frac{I F}{I F-1}
$$

Assuming IF $\approx 5$ for design margin, the inverter delay evaluates to $\frac{1}{2}(40 p s) \cdot \frac{5}{4}=$ 25ps. To provide some design margin, it would be wise to make it even smaller. 
However, achieving faster propagation delay in this $0.13 \mu \mathrm{m}$ technology may not be feasible.

Therefore, an alternative solution is to target lower IF for the same $t_{d}^{\text {gate }}=25 \mathrm{ps}$. From TDC specification (Section 4.3 the $\mathrm{T}_{\mathrm{LSB}}=8 \mathrm{ps}$, which evaluates to 3 (5.4).

$$
I F=\frac{t_{d}^{\text {gate }}}{T_{L S B}}=\frac{25 p s}{8 p s} \approx 3
$$

Therefore, the adjusted inverter specification is $\mathrm{t}_{\mathrm{d}}^{\text {gate }}=24 \mathrm{ps}, \mathrm{IF}=3$.

To ensure simulation accuracy at high frequencies, the RF models of n-type MOSFET (NFET) and p-type MOSFET (PFET) devices are used. The improved model accuracy compared to standard MOSFET transistor models comes at the cost of layout placement restrictions and increased minimum gate widths. However, such trade-offs are deemed reasonable, given the experimental nature of the work, which depends on simulation results. The inverter delay unit is shown in Figure 5.5

Figure 5.5: The Inverter Delay Unit schematic showing RF MOSFET transistors. 
To maintain overall linearity of time-to-digital converter, both high-low and lowhigh transfer characteristics should have equal delays (i.e. same switching point). Ideally this transition should happen at an input signal level of $\approx \frac{V D D}{2}$, where VDD is the supply voltage. This condition requires that the inverter should have equal drive strengths for charge and discharge currents, the PFET and NFET are sized such that $\frac{W_{P}}{L_{P}}=\frac{\mu_{N}}{\mu_{P}} \cdot \frac{W_{N}}{L_{N}} \approx 3 \cdot \frac{W_{N}}{L_{N}}$, where $\mu_{P}$ and $\mu_{N}$ represent carrier mobility for a given FET, which is a process parameter specific to technology. The device sizes are iteratively adjusted through simulation until desired propagation delay is achieved, while maintaining sizing ratio for symmetrical switching. The final device sizes are given in Table 5.1 .

Table 5.1: Differential delay unit (inverter) device sizing.

\begin{tabular}{cccc}
\hline Device & Width $\mu \mathrm{m}$ & Length $\mathrm{nm}$ & Fingers \\
\hline T0 & 15.9 & 120 & 15 \\
T2 & 48.6 & 120 & 15 \\
\hline
\end{tabular}

Inverter delay varies over process corners, supply voltage, and temperature. An additional margin was built into inverter drive strength to make sure that $\mathrm{t}_{\mathrm{d}}^{\text {gate }}=24 \mathrm{ps}$ can be achieved at voltages much lower than maximum allowable of $\mathrm{VDD}=1.5 \mathrm{~V}$ and cover a wide range of temperatures as seen in Figure 5.6 The process corner was assumed to be close to nominal for this demonstration, based on historical data from the manufacturer. From Figure 5.6 it is expected that at room temperature, the $T_{L S B} \leq 24 p s$ can be achieved over the enire range of VDD levels. However, due to self-heating and finite thermal resistance of packaging, the final junction temperature will be higher. At $100{ }^{\circ} \mathrm{C}$, supply voltage will have to be $>1.4 \mathrm{~V}$.

The falling and rising delay mismatch is kept $<1$ ps by design within expected region of VT operation. This translates to DNL error of $<0.04 L S B \approx \frac{1 p s}{3 \cdot 8 p s}$. Moreover, 


\begin{tabular}{|c|c|c|}
\hline Name & Vis & vdd \\
\hline \multicolumn{3}{|l|}{ t_delay_rising } \\
\hline t_delay_rising & a & 1.2 \\
\hline t_delay_rising & a & 1.3 \\
\hline t_delay_rising & a & 1.4 \\
\hline t_delay_rising & a & 1.5 \\
\hline \multicolumn{3}{|l|}{ t_delay_falling } \\
\hline $\mathrm{t}$ delay falling & a & 1.2 \\
\hline t_delay_falling & a & 1.3 \\
\hline t_delay_falling & a & 1.4 \\
\hline t_delay_falling & a & 1.5 \\
\hline
\end{tabular}

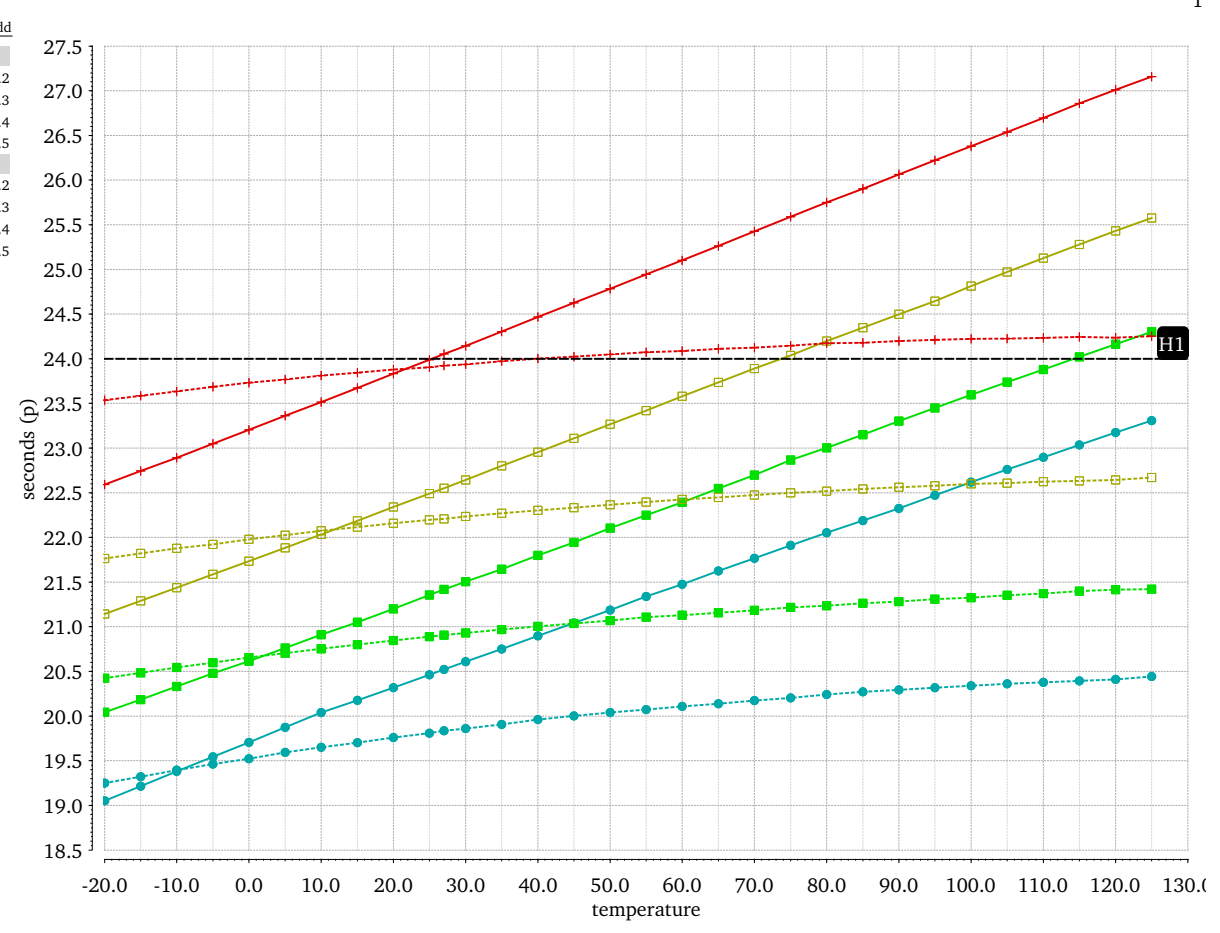

Figure 5.6: Inverter delay over VT variation (nominal process corner).

the mismatch does not result in growing skew, benefiting from a differential structure of the LPI-TDC interpolator design as mismatches cancel from one delay stage to another, as rising and falling edge interpolation switches sides.

The differential delay unit layout is in Figure 5.7 Each inverter benefits from a low-impedance double-sided gate ring to make sure all device fingers carry equal current, thus maintaining the device characteristic predicted by the model. The builtin guard rings for PFET and NFET devices also help alleviate latch-up concerns (for design reliability). Thick power (VDD) and ground (VSS) rails were implemented using stacked parallel metal plates uniformly strapped through via arrays across entire area. This was done to ensure that a large amount of dynmaic power can be delivered unaffected by low supply impedance. The power rails were designed to handle $50 \mathrm{~mA}$ of DC current. The estimated dynamic current draw by LPI-TDC core is on the order of $50 \mathrm{~mA}$. In this technology the electromigration rules estimate a ratio of $I_{R M S} \approx I_{D C}$, 


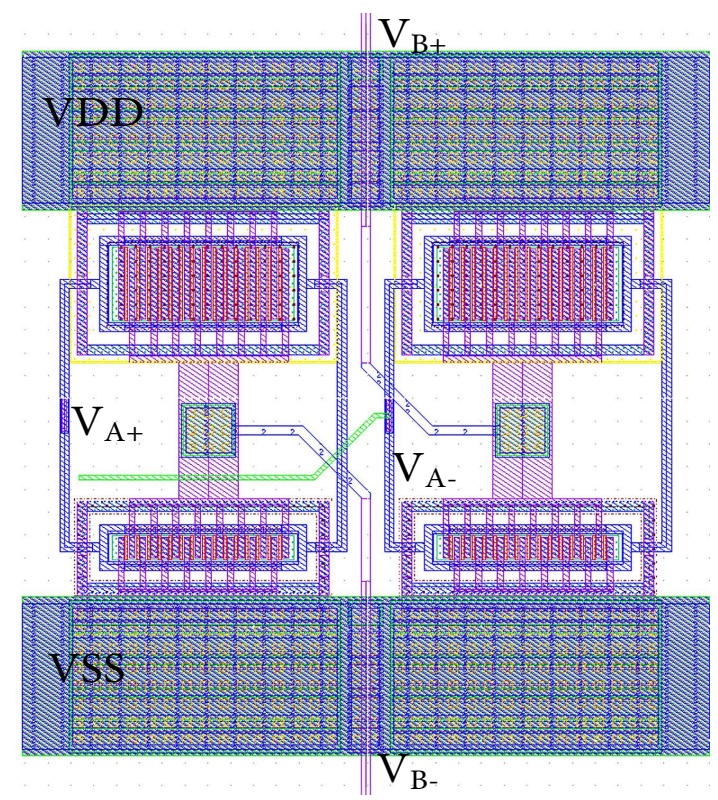

Figure 5.7: TDC delay unit layout.

clearly showing healthy margin to accommodate larger currents if required. .

Using this delay unit layout arrangement, the complete delay cell including resistive interpolator was assembled. The resistors were sized such that they occupy the same horizontal footprint as differential delay unit, as well as to be able to handle large cross currents. The final dimensions of interpolation are in Table 5.2

Table 5.2: Interpolation resistor sizing.

\begin{tabular}{ccccc}
\hline Device & Width $\mu \mathrm{m}$ & Length $\mu \mathrm{m}$ & Multiplicity & Resistance $[\Omega]$ \\
\hline $\mathrm{R}_{\mathrm{INT}}$ & 8 & 1.68 & 2 & 47.6 \\
\hline
\end{tabular}

Two of such interpolation delay cells were abutted together in layout and subsequently extracted for post-layout simulations. However, the extracted views generate large netlists which significantly slow down simulations. To speed up the post-layout verification without losing the modelling accuracy, dominant parasitics were backannotated from extracted view to schematic circuit as shown in Figure 5.8 Block-level 
verification was performed to ensure that responses for extracted and back-annotated schematic netlists match.

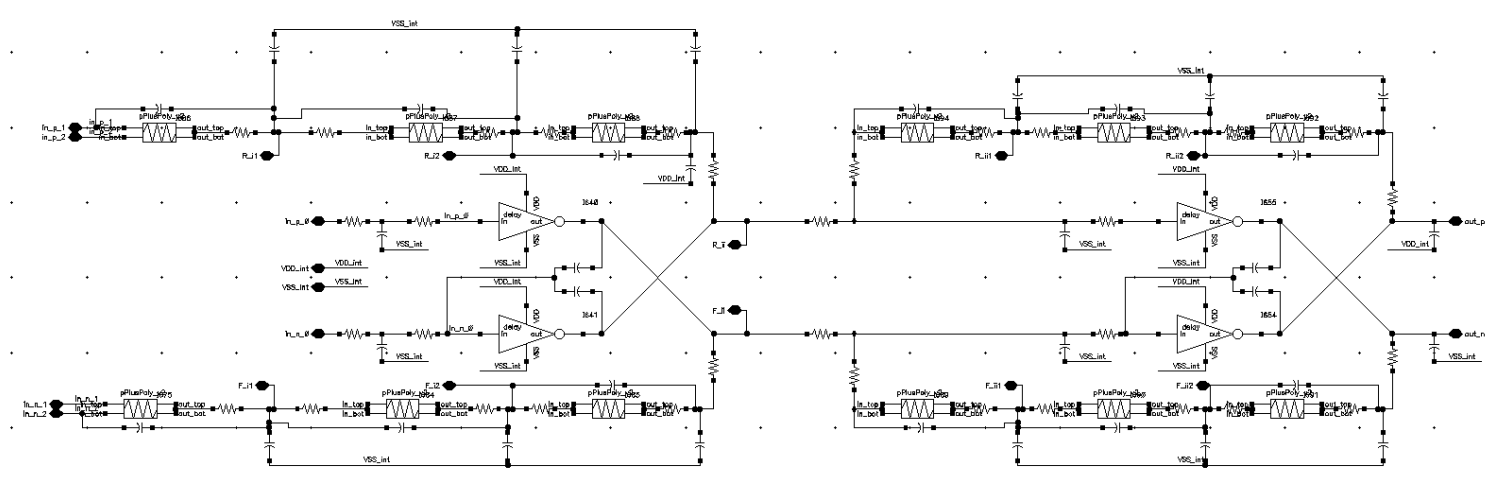

Interpolation resistors unit 1 Interpolation resistors unit 2

$\begin{array}{lllllll}\mathrm{V}_{\mathrm{A} 1+} & \mathrm{V}_{\text {int2+ }} & \mathrm{V}_{\text {int3+ }} & \mathrm{V}_{\mathrm{B} 1+} & \mathrm{V}_{\text {int5+ }} & \mathrm{V}_{\text {int6+ }} & \mathrm{V}_{\mathrm{B} 2+} \\ \text { VDD } & & & & & \end{array}$
$\mathrm{V}_{\mathrm{A} 1+} \quad$ differential delay unit $1 \quad$ differential delay unit 2 $\mathrm{V}_{\mathrm{A} 1-}$

VSS
$\mathrm{V}_{\mathrm{A} 1-} \quad \mathrm{V}_{\text {int2- }} \quad \mathrm{V}_{\text {int3- }} \quad \mathrm{V}_{\mathrm{B} 1-} \quad \mathrm{V}_{\text {int5- }} \mathrm{V}_{\text {int6- }}$

Figure 5.8: Dual delay stage schematic with back-annotated parasitic capacitances and resistances for simulation accuracy. 


\subsection{Interpolation Core}

Using the interpolation delay cells discussed in Section 5.1, the core is assembled. The minimum target period for proposed fine LPI-TDC is $\frac{1}{5 G H z}=200 p s$. However, it is worth extending the range, to account for TDC resolution variation. From preliminary simulations of interpolation delay cell (Figure 5.6), a minimum resolution of $\approx 7 p s$ is expected. A 5-bit TDC was set as target specification (Section 4.3) agrees with simulation predictions so far and should be able to accommodate the full start signal period $D R=7 p s \cdot 2^{5}=224 p s$. However, the first and last interpolation nodes will experience different capacitive loading, which could lead to linearity degradation. Therefore, additional interpolation nodes are required to pad (buffer) the first and last bits of the interpolator. A total of 11 delay cells were placed, taking up $312 \mu \mathrm{m} \times 48 \mu \mathrm{m}$ of the chip area $\left(0.015 \mathrm{~mm}^{2}\right)$. Of the 34 nodes available, the middle $2^{5}=32$ are expected to be used by the converter. The resulting offset between the start and the stop signals due to the unused nodes is deterministic and can be calibrated in the post-processing if required.

The complete interpolator core layout is shown in Figure 5.9

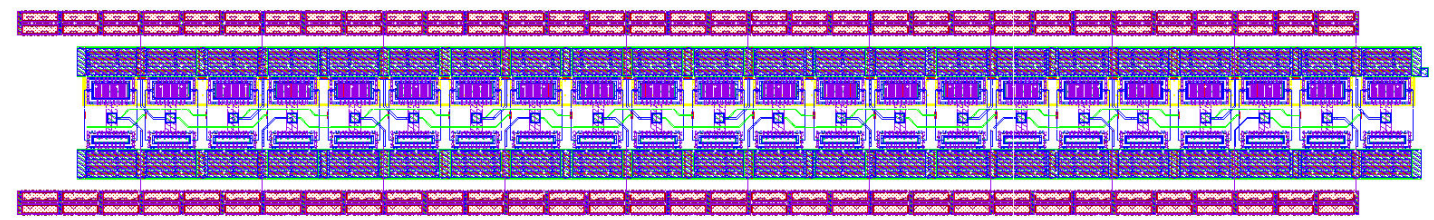

Figure 5.9: Interpolation core of 5-bit LPI-TDC consisting of 11 individual delay cell units with interpolation factor $\mathrm{IF}=3$ each. 


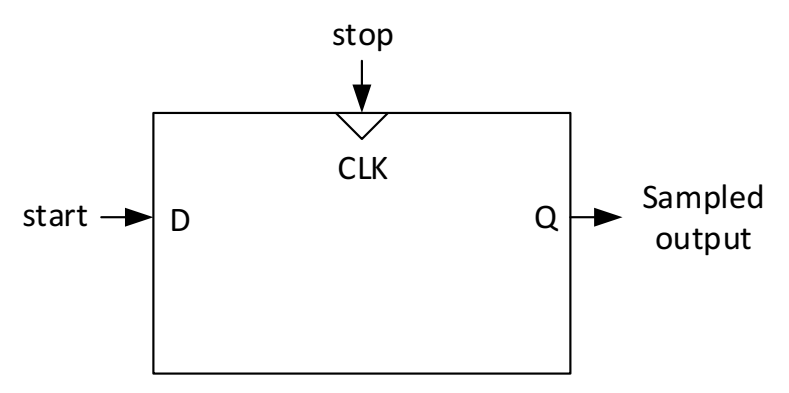

Figure 5.10: A basic Flip-Flop block diagram.

\subsection{Sense Amplifier Assisted Flip-Flop}

The reference clock (stop) captures the state of interpolated input (start) signal. The output of interpolator appears as a monotonic pseudo-thermometer code switching at rate of start signal. For this design, it was decided to use on-chip state sampling in order to avoid routing entire 5-bit wide output (32 nodes) while switching at $5 \mathrm{GHz}$, since it would pose a significant signal integrity challenge in measurement. Therefore, a Flip-Flop structure was chosen to sample the LPI-TDC interpolator output state at lower rate of $\approx 100 M H z$ as per specification (Section 4.3). A generic Flip Flop diagram is shown in Figure 5.10 to establish terminology in the context of TDC discussion. The input Data $(D)$ is sampled by clock $(C L K)$ on rising edge. The result of sampling $D$ by $C L K$ is sent to output terminal $Q$.

The conventional digital style rise-edge Flip-Flop suffers from large setup/hold time requirements, usually on the order of tens of picoseconds 4,22 . The asynchronous nature of LPI-TDC state sampling has to take into account that input data $D$ might be in the middle of transition when the rising edge of sampling clock arrives. This would clearly violate the setup time requirements and force Flip-Flop to operate in meta-stable state. If the region of sampling uncertainty is very wide, far exceeding the $\mathrm{T}_{\mathrm{LSB}}$ resolution, multiple bits of the interpolation core may resolve to an incorrect value and most importantly cause a non-monotonic thermometer code. 
Errors of this type are typically referred to as "bubble errors" and their effect on the resulting TDC digital word is shown in a hypothetical example Table 5.3

Table 5.3: Flip-Flop sampling uncertainty causing bubble errors in TDC output word.

\begin{tabular}{lrr}
\hline scenario & thermometer code (32LSB, i.e. 5-bit) & TDC output code \\
\hline 0 bubble errors & 1111111111000000000000000000000 & 10 \\
1 buble error & 11111111110100000000000000000000 & 12 \\
2 bubble errors & 11111111110101000000000000000000 & 14 \\
\hline
\end{tabular}

Taking advantage of differential LPI-TDC interpolation core operation, the decision was made to use a Sense-Amplifier assisted Flip-Flop (SAFF) as seen in Figure 5.11 23], which are commonly found in RAM memory designs 22 . These circuits are also known as tactical flip-flops in some literature [4]. The use of digital style pre-amplifier minimizes effective setup time requirement due to its superior voltage sensitivity. The topology of the sense amplifier also makes the latch timing independent of input polarity, since all sense nodes are properly discharged after each comparison. The Set-Reset latch performs the state capture until the arrival of the next clock (stop ) rising edge.

Although improved SAFF performance over a standard Flip-Flop doesn't guarantee complete avoidance of bubble errors, it can limit the issue to a manageable degree. Any bubble errors caused by zero-mean random processes could potentially be dealt with averaging the TDC output code, which is inherently expected in an ADPLL application. However, bubble errors caused by systematic circuit issues (e.g. kick-back, clock feed-through) require different techniques. For such cases, there are well-known algorithms such as priority encoders which can detect and correct bubble errors $[22$. Adding error-correction capability comes at a cost of increased system complexity, 


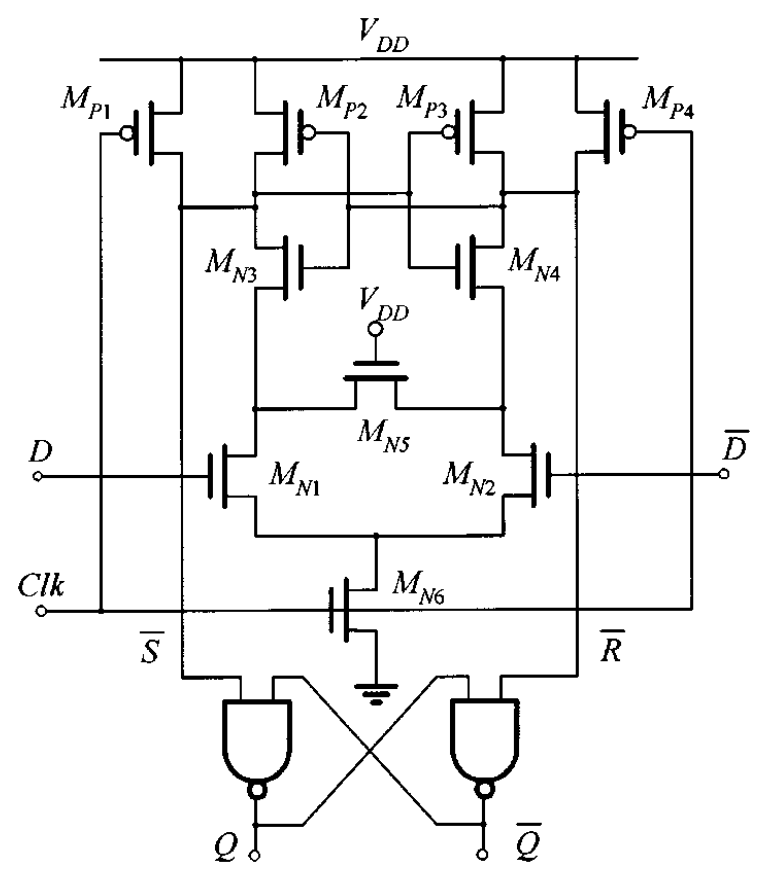

Figure 5.11: Sense-Amplifier assisted Flip-Flop.

power, and chip area. Furthermore, most error-correction solutions scale exponentially 24,25 with number of concurrent bubble errors that need to be removed within a single clock cycle. Therefore, for practical bubble error correction design, it was decided to limit the number of concurrent bubble errors to $<2$ LSB (ideally $<1$ LSB) of TDC resolution.

The optimization method proposed in [26] was used to minimize the latch delay and increase sensitivity (slew rate). The sense amplifier performance was optimized around $\frac{V D D}{2}$ input level, to match the interpolator output characteristic. The differential input pair was also deliberately sized larger to minimize mismatches that can cause offsets. The final SAFF schematic is seen in Figure 5.12

A significant amount of effort went into creating a well-matched layout while minimizing the parasitic capacitances that could affect the timing. To minimize offsets, devices were split into multiple fingers and placed in common-centroid fashion as well 


$\begin{array}{cccc}\text { M7 } & \text { M2 } & \text { M4 } & \text { M8 } \\ \text { M1 } & \text { M3 } & \\ & & \\ \text { M5 } & \text { M6 } & \text { M9 }\end{array}$

M21

M23

M22

M24

$\begin{array}{cccc}\text { M0 } & \text { M10 } & \text { M14 } & \text { M12 } \\ & & & \\ & \text { M11 } & \text { M13 } & \\ \text { M17 } & \text { M15 } & \text { M20 } & \text { M19 } \\ & & & \\ & & & \\ & \text { M16 } & \text { M18 } & \end{array}$

Figure 5.12: Sense-Amplifier assisted Flip-Flop.

as applying inter-digitation technique to uniformely distribute all critical devices, such as M5,M6 (Figure 5.13). An additional effort was made to layout this cell that would suit digital standard cell placement making it suitable for abutting adjacent cells as well as vertical pitch approximately equal to the interpolator delay cell.

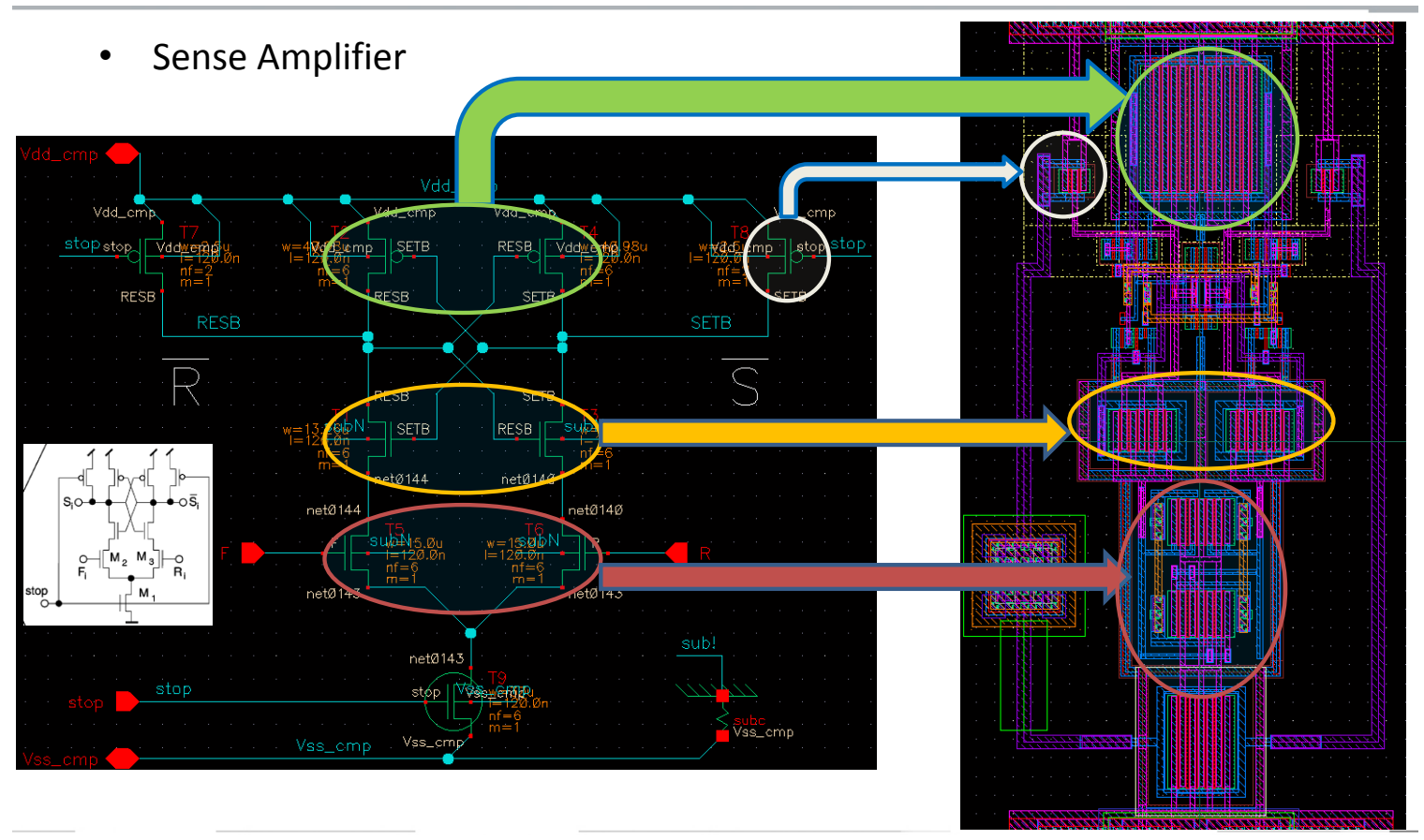

Figure 5.13: Sense-Amplifier common-centroid and inter-digitated layout of input differential pair.

The layout was ultimately extracted in a similar the same way as interpolator 
delay cell, and parastic capacitances were back-annotated to schematic for subsequent simulation. The post-layout verification results show the simulated sense amplifier behaviour in Figure 5.14. Two important phases can be identified in Figure 5.14 $t_{o}$ and $t_{l a t c h}$. The $t_{o}$ is defined as time from arrival of the clock signal to the point when M2 transistor turns on - this is the time required to begin the latching process. The $t_{\text {latch }}$ is the second phase during which the latching occurs, which is the most critical timing from LPI-TDC point of view, because any offsets, noise and other impairments during this time could compromise the decision. The SAFF design was optimizied to minimize both delays.

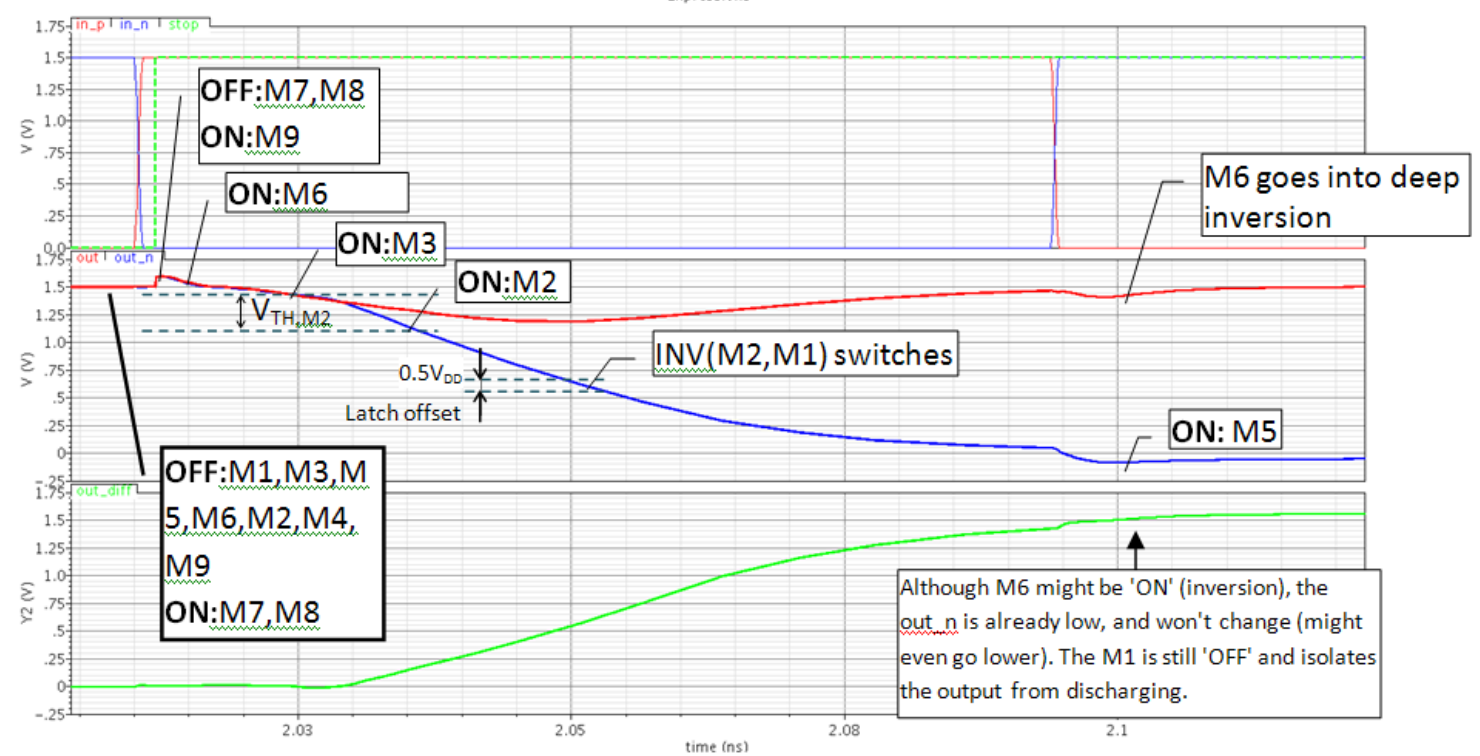

Figure 5.14: SAFF transient behaviour showing conversion sense amplifier in action with small CLK-to-D delay applied at the input.

To ensure that mismatches don't cause major degradation in $t_{\text {latch }}$ timing, a Monte-Carlo simulation was performed with over 500 runs to increase the confidence level Figure 5.15. The value of $t_{\text {latch }} \approx 10.8 p s$ is equivalent to $\frac{t_{\text {latch }}}{T_{L S B}}=\frac{10.8 p s}{8 p s}=$ 1.35LSB, which is $<2 \mathrm{LSB}$ as required. 
tlatch_SwitchAtop5Vdd_27

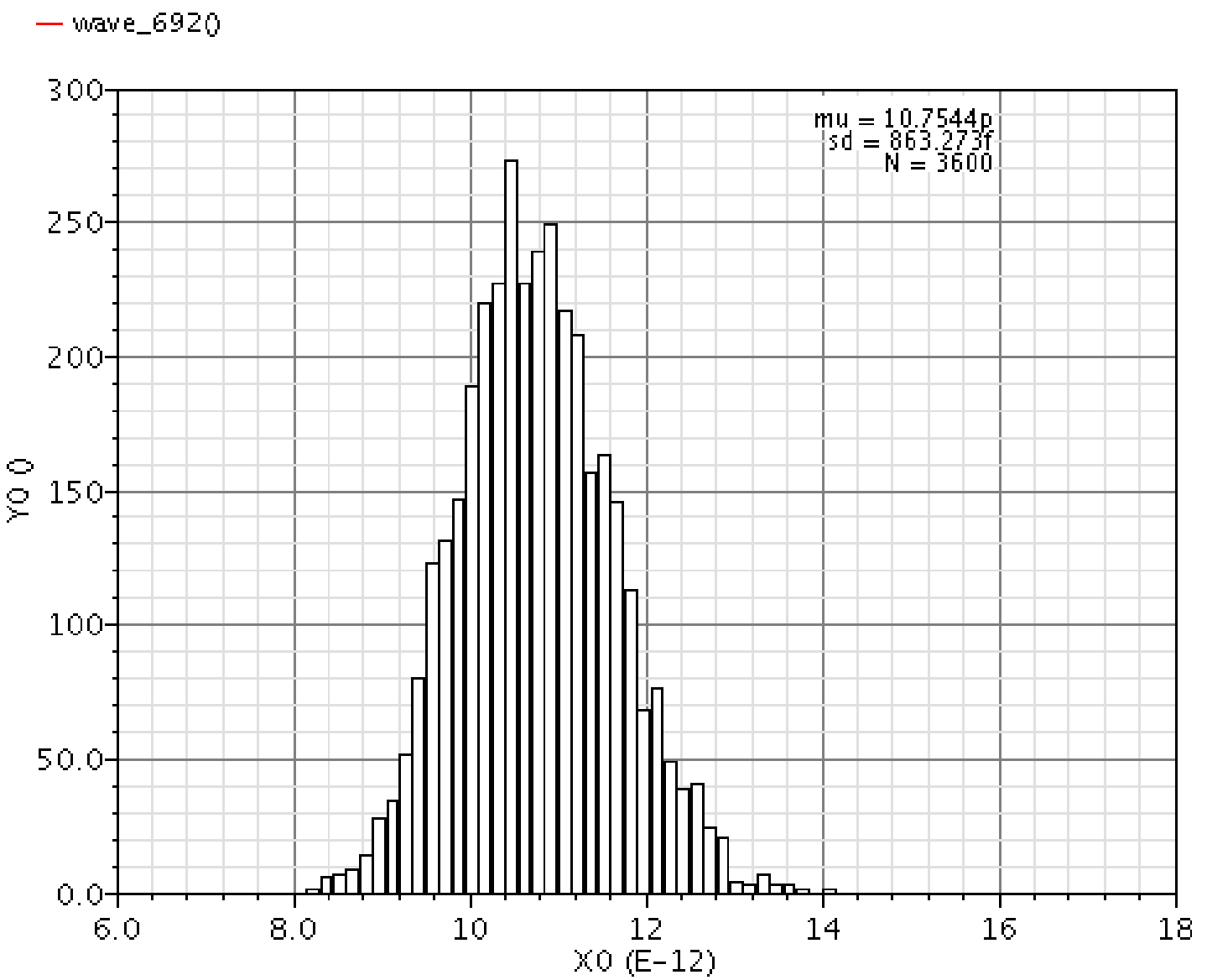

Figure 5.15: SAFF $t_{\text {latch }}$ timing variation due to design offsets caused by device mismatches.

An additional check was performed to verify the overall SAFF timing, ensuring that the maximum delay does not exceed the expected clock period of $10 \mathrm{~ns}$. It was also done to verify that the sampling window uncertainty does in fact remain $<2$ LSB when paired with the RS latch (which should be the case, given that it is dominated by SA stage sensitivity). The resulting plot in Figure 5.16 confirms the suitability of this SAFF to handle LPI-TDC latching. The window of uncertainty can be observed around the 55-65 ps mark when CLK-to-Q delay sharply increases. The overall width 


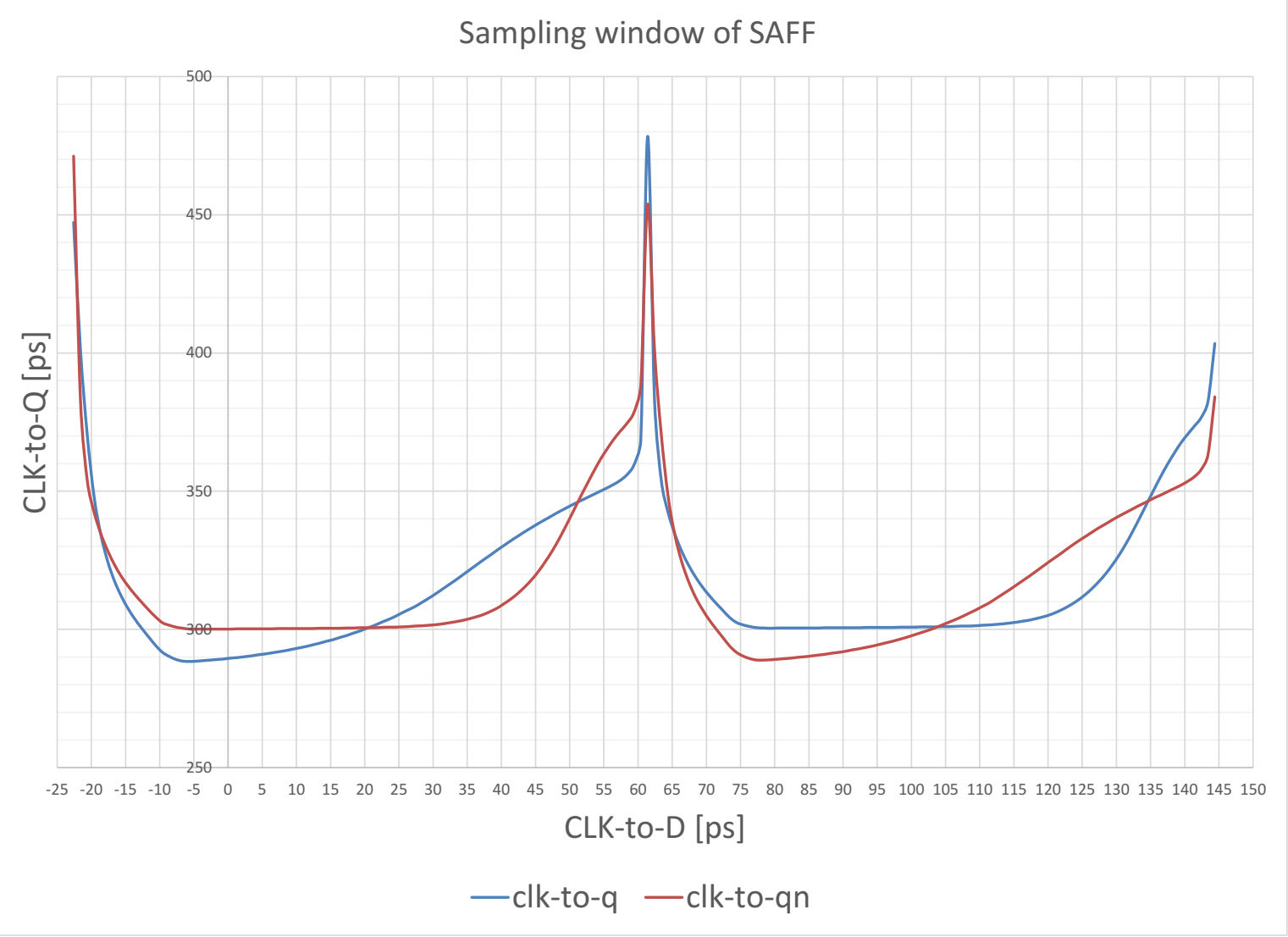

Figure 5.16: SAFF CLK-to-Q delay as a function of CLK-to-D delay shows very wide sampling window suitable for LPI-TDC resolution.

of the peak is about $10 p s=1.25 L S B$ in terms of CLK-to-D delay as expected.

Optimized device sizes are given in Table 5.4

Table 5.4: Device size specifications for the SAFF.

\begin{tabular}{cccc|cccc}
\hline Transistor & Width $\mu \mathrm{m}$ & Length $\mathrm{nm}$ & Fingers & Transistor & Width $\mu \mathrm{m}$ & Length $\mathrm{nm}$ & Fingers \\
\hline M9 & 30 & 120 & 6 & M15=M20 & 0.5 & 120 & 1 \\
M5=M6 & 15 & 120 & 6 & M16=M18 & 0.5 & 120 & 1 \\
M1=M3 & 13.26 & 120 & 6 & M17=M19 & 1 & 120 & 1 \\
M2=M4 & 40.98 & 120 & 6 & M0=M12 & 2.24 & 120 & 2 \\
M7=M8 & 2.5 & 120 & 2 & M21=M22 & 320 & 120 & 1 \\
M11=M13 & 1.12 & 120 & 1 & M23=M24 & 640 & 120 & 1 \\
M10=M14 & 1.12 & 120 & 1 & & & & \\
\hline
\end{tabular}


In most practical designs, the TDC thermometer code is further encoded (compressed) into binary format to reduce the number of bits for subsequent computations. This also simplifies routing, reduces chip area, power, and complexity of the circuitry outside of the TDC block. However, to minimize the risk of on-chip implementation errors, the thermometer-to-binary encoding and bubble error-correction of TDC output was intentionally assume off-chip implementation. Leaving out the onchip encoders allows for greater flexibility in measurements and unforeseeable debug.

\subsection{Signal Buffers}

Buffers are designed to drive large capacitive loads and to provide isolation between circuits. Several locations in the design have been identified to require such buffers: complimentary start input signals, output of Sense Amplifier Flip-Flops (SAFF), as well as the interface between interpolator core and SAFF. The start signal was left intentionally unbuffered, since it is running at relatively low frequencies and can be easily driven by off-chip sources. Such choice also enables options for bench-level experimentation with sampling clock amplitudes and timing.

All driver interfaces follow the minimum propagation delay tapered digital buffer design methodology [22]. The general principle is to cascade inverters with increasing drive strength (device sizes) in order to minimize the overall propagation delay as seen in Figure 5.17

The ratio of PFET to NFET widths $\frac{W_{p 1}}{W_{n 1}}$ (assumes same gate length) for the first element in the chain is scaled by a factor $\mathrm{A}^{\mathrm{N}}$, where $\mathrm{N}$ stands for inverter index. Mathematically the optimal delay can be achieved if the scale factor A is sized according to (5.5) [22].

$$
A=\left(\frac{C_{\text {out }}}{C_{\text {in }}}\right)^{\frac{1}{N}}
$$




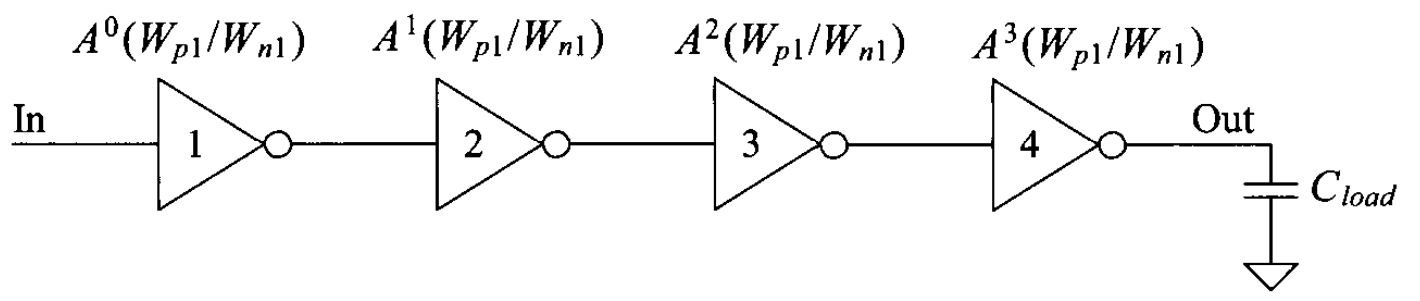

Figure 5.17: Cascaded inverters used to drive large capacitive load 2.

For CMOS style digital circuitry, the optimal solution is approximated by 5.6, where scaling factor $A=e \approx 2.72$. This assumes that the inter-stage wiring impedance is ignored.

$$
N=\ln \left(\frac{C_{\text {out }}}{C_{\text {in }}}\right)
$$

All peripheral interfaces also include parasitic inductance to model package bondwires, which was estimated $\approx 1 n H[5]$. From system level perspective, the additional delays added by all buffers result in phase offset between start and stop signals. However, the resulting output code offset can be simply calibrated during measurements.

\subsubsection{Input Buffer}

The input buffer for start signals was used to handle RMS cross-currents caused by interpolator switching. Such choice was driven by an uncertainty as to what equipment would be used in measurements, so an on-chip buffer was used to drive the interpolator at $5 \mathrm{GHz}$. To simplify the analysis, a buffer with the last stage being the same as the interpolator delay unit was chosen. The first stage was sized as a minimum-size symmetrical inverter. A quick calculation using (5.5) and (5.6) as a guide resulted in a buffer design seen in Figure 5.18 The main goal is to ensure that the overall phase offset stays well within one period of stop signal to avoid any additional measurement complications. 
Figure 5.18: Cascaded inverters used to drive interpolator core. $\mathrm{A} \approx 2.44, \mathrm{~N}=5$ stages.

For modelling accuracy, a 1nH bond-wire inductance was used. A set of primary and secondary electro-static discharge (ESD) protection diodes and bond-pad equivalent circuit were also included to account for their contribution to input parasitic capacitance. The ESD structure designed to protect against human-body model (HBM) discharge event was used to improve chip reliability in bench measurements and handling.

The buffer device sizes are summarized in Table 5.5 All devices have the same drawn gate length of $120 \mathrm{~nm}$.

Table 5.5: Device sizes of input buffer stages.

\begin{tabular}{cccccc}
\hline Stage index $(\mathrm{N})$ & $\mathrm{W}_{\mathrm{P}} \mu \mathrm{m}$ & $\mathrm{W}_{\mathrm{N}} \mu \mathrm{m}$ & $\mathrm{NOF}_{\mathrm{P}}$ & NOF $_{\mathrm{N}}$ & stage scaling $\left(\mathrm{A}^{\mathrm{N}}\right)$ \\
\hline 1 & 1.23 & 0.48 & 1 & 1 & 1 \\
2 & 3.24 & 1.14 & 4 & 2 & 2.44 \\
3 & 7.95 & 2.75 & 5 & 5 & 5.73 \\
4 & 19.9 & 6.6 & 10 & 10 & 13.75 \\
5 & 48.6 & 15.9 & 15 & 15 & 33.13 \\
\hline
\end{tabular}

\subsubsection{Interpolator to SAFF Buffer}

The SAFF circuit was designed to have fast charge/discharge current handling capabilities to achieve high sensitivity. However, this comes at a cost of increased load capacitance presented to the interpolator core. From earlier discussion in Section 5.1 the inteprolator load capacitance budget was assumed to be «100 fF. Therefore, a 
buffer would be useful to isolate the delay cell output from the SAFF input capacitance. Another reason to place a buffer at this interface is to reduce any clock feedthrough and kick-back during SAFF latching, which can cause a significant amount of undesired disturbance at interpolator output nodes.

To simplify the design task for this buffer, it was decided to reuse the existing blocks that were utilized in input buffer implementation Section 5.4.1. The threestage design is shown in Figure 5.19 Device sizes are identical to the first three stages of input buffer and can be found in Table 5.5
$\mathrm{N}=1$
$\mathrm{N}=2$
$\mathrm{N}=3$

Figure 5.19: Cascaded inverters used to isolate interpolator core from the SAFF. $\mathrm{A} \approx 2.44, \mathrm{~N}=3$ stages.

\subsubsection{Output Buffer}

The output of the SAFF has to drive package parasitic capacitances, as well as any instrumentation attached to it, such as a logic analyzer or oscilloscope probes. The combined load capacitance was estimated at 10pF. A Bond-pad model and a bondwire inductance were also included in verification. However, since the output is operating at a stop rate of $\approx 100 M H z$, the main requirement for this buffer is to ensure that the delay is «10 ns. Therefore, to save power and chip area, a single stage design 
Table 5.6: Device sizes of output buffer stages.

\begin{tabular}{|c|c|c|c|c|c|}
\hline Stage index $(\mathrm{N})$ & $\mathrm{W}_{\mathrm{P}} \quad \mu \mathrm{m}$ & $\mathrm{W}_{\mathrm{N}} \quad \mu \mathrm{m}$ & $\mathrm{NOF}_{\mathrm{P}}$ & $\mathrm{NOF}_{\mathrm{N}}$ & stage scaling $\left(\mathrm{A}^{\mathrm{N}}\right)$ \\
\hline 1 & 48 & 24 & 12 & 12 & $\approx 20$ \\
\hline
\end{tabular}

was used, summarized in Table 5.6 .

\subsection{Top-Level Integration}

The assembled chip ICGCUKIK is shown in Figure 5.20. The overall chip dimensions are $1.5 \mathrm{~mm} \times 1.5 \mathrm{~mm}$, while the LPI-TDC, SAFF, and core buffers take up $367 \mu \mathrm{m} \times$ $242 \mu \mathrm{m}\left(0.09 \mathrm{~mm}^{2}\right)$.

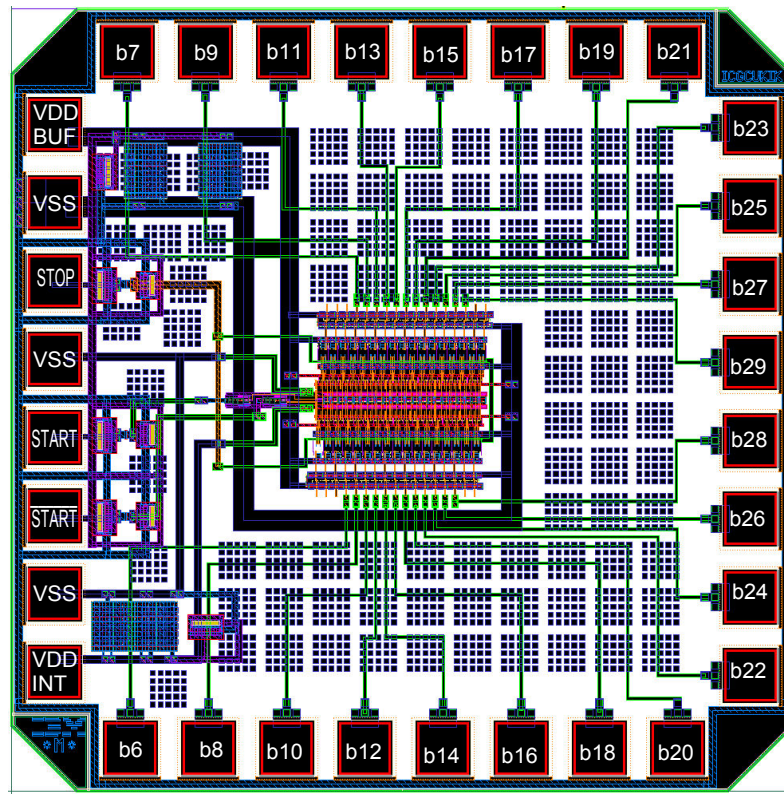

(a) chip layout

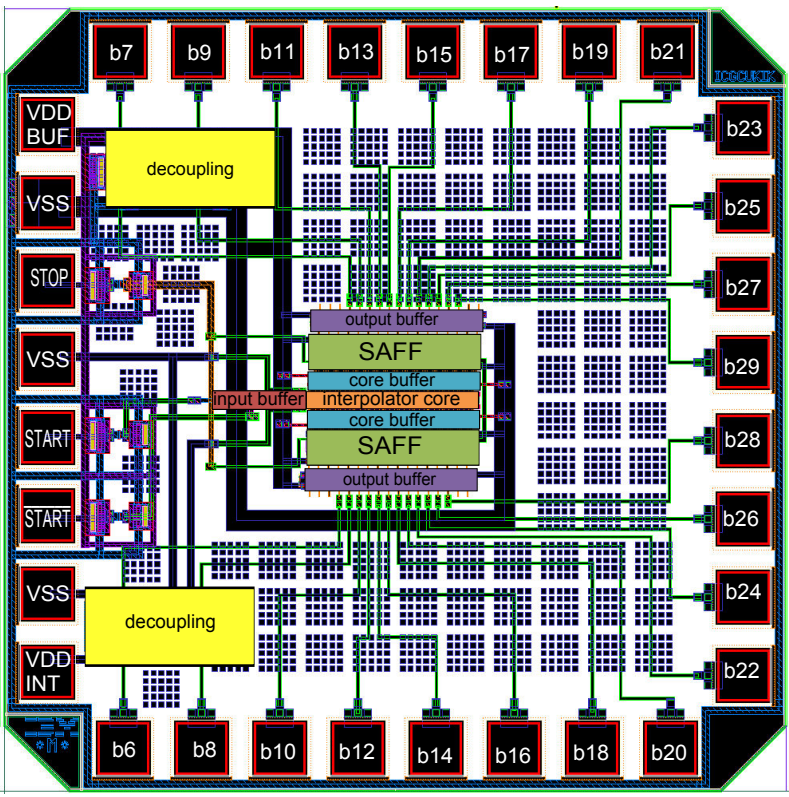

(b) chip floorplan

Figure 5.20: ICGCUKIK, LPI-TDC layout with metal fill.

Instead of connecting all 32 bit lines $\left(=2^{5}\right)$ of the LPI-TDC output, only a subset of 24 bit lines (bit $<6>$ to bit $<29>$ ) were routed to the chip interface. The decision was mainly driven by schedule limitation, because designing a new package with 
dimensions suitable to handle all 32 bit lines was not feasible at the time of tape-out. However, this does not limit the ability to characterize device FOM and performance metrics, as will be discussed later in Chapter 6 .

To avoid the noise coupling (switching, kick-back, clock feedthrough) between the SAFF and the interpolator core, each block has been intentionally assigned a separate power domain (VddBuf and VddInt respectively). The VddBuf supply was also used to power the chip output buffers. The grounds had to be shorted together on the chip due to the limited number of available bond-pads, which makes this choice less than ideal for noise coupling.

The reactive impedance of supply traces was also taken into consideration. This is especially important for digital design such as LPI-TDC, given the dynamic switching power consumption of the device. To provide instantaneous charge and reduce the supply impedance at higher frequencies, some decoupling capacitors were added to the design in the form of NCAP varactor devices. The type of decoupling element was selected based on the highest capacitance density per area of all devices available in this technology. The VddBuf has $176 \mathrm{pF}$ of on-chip decoupling, while the VddInt has $160 \mathrm{pF}$. All supply traces have been designed to handle expected current densities at $5 \mathrm{GHz}$ (see Table 5.7). Capacitive coupling between power domains and signals is minimized by physical separation, routing in different metal layers, and making all trace crossings perpendicular to minimize the overlap area.

\subsection{Post-Layout Design Verification}

The top-level routing was extracted and added to the back-annotated schematic models that have been previously matched by performance with the block-level extracted views. This approach saved many hours of simulation, cutting a single transient 
simulation run from 48 hours using fully-extracted view, down to $\approx$ 4hours using back-annotated schematics. However, even with optimized simulation effort, certain metrics such as SSP were left to be verified in measurements. The resolution at 5 $\mathrm{GHz}$ was measured directly at the interpolator core, by measuring an average delay observed across the delay chain. The LPI-TDC conversion latency from arrival of the stop rising edge to the point when the code is available was also verified to be «10 ns Figure 5.21 .

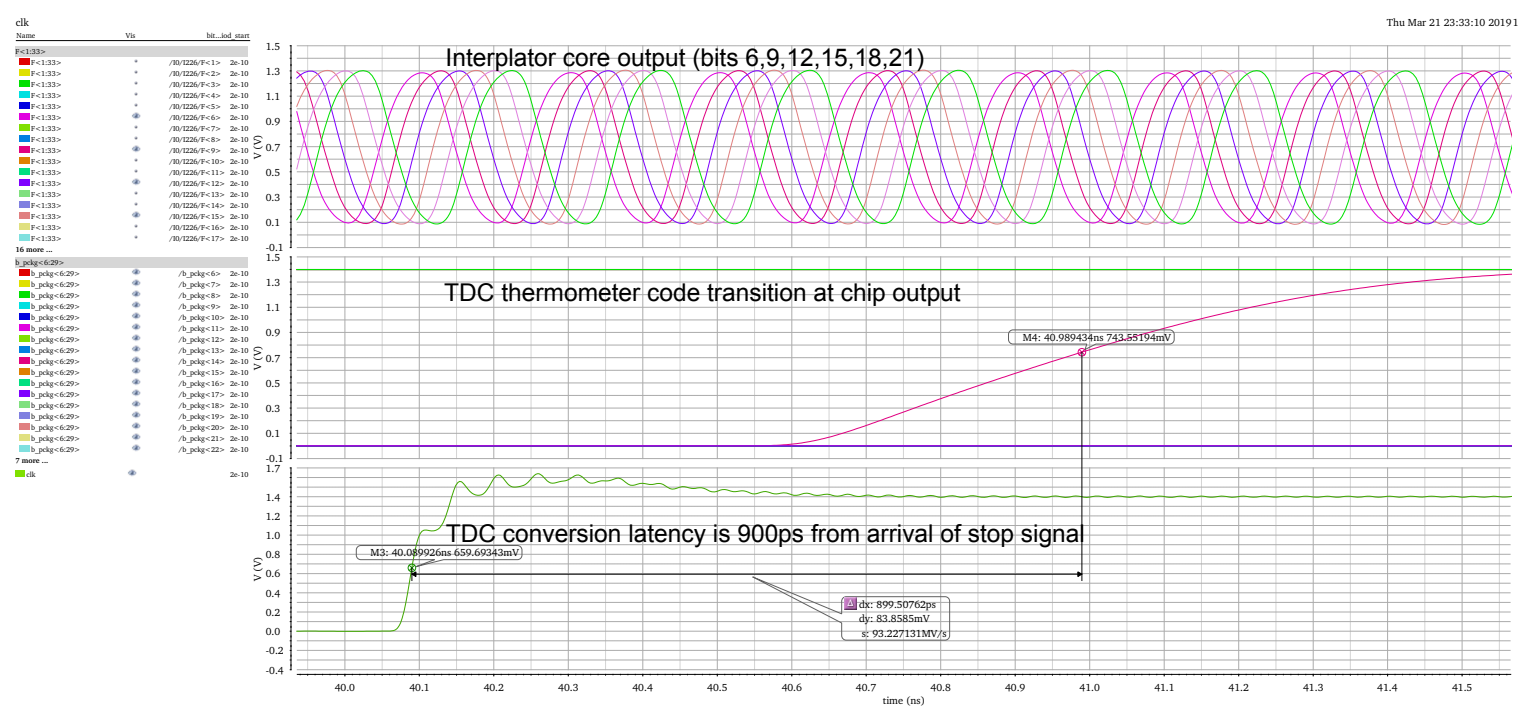

Figure 5.21: Simulated LPI-TDC interpolator core and system latency. Conditions: $\mathrm{f}_{\text {start }}=5 \mathrm{GHz}, \mathrm{f}_{\text {stop }}=100 \mathrm{MHz}, \mathrm{VDD}=1.4 \mathrm{~V}, \mathrm{~T}=65^{\circ} \mathrm{C}$.

To measure non-linearity, a phase offset between start and stop (=testperiod) was swept. The resulting TDC thermometer code was recorded and converted to decimal representation. To speed up the simulation process, the non-linearity had to be measured at $1 \mathrm{GHz}$, keeping all other conditions unchanged. The nominal temperature was selected to be $65^{\circ} \mathrm{C}$ to take into account self-heating of the device in application at ambient room temperature of $27^{\circ} \mathrm{C}$. The INL of $0.86 \mathrm{LSB}$ and DNL of 0.43 LSB was observed. Both quantities are within specification to ensure robust operation. However, the coarse test period step size of 5 ps is larger than the maximum 
allowed for accurate assessment using the code density method (Section 4.1.3), which requires the step size to be step $\leq \frac{1}{10} T_{L S B} \approx 0.8 p s$. Therefore, simulation results are expected to show even better linearity with finer step size.

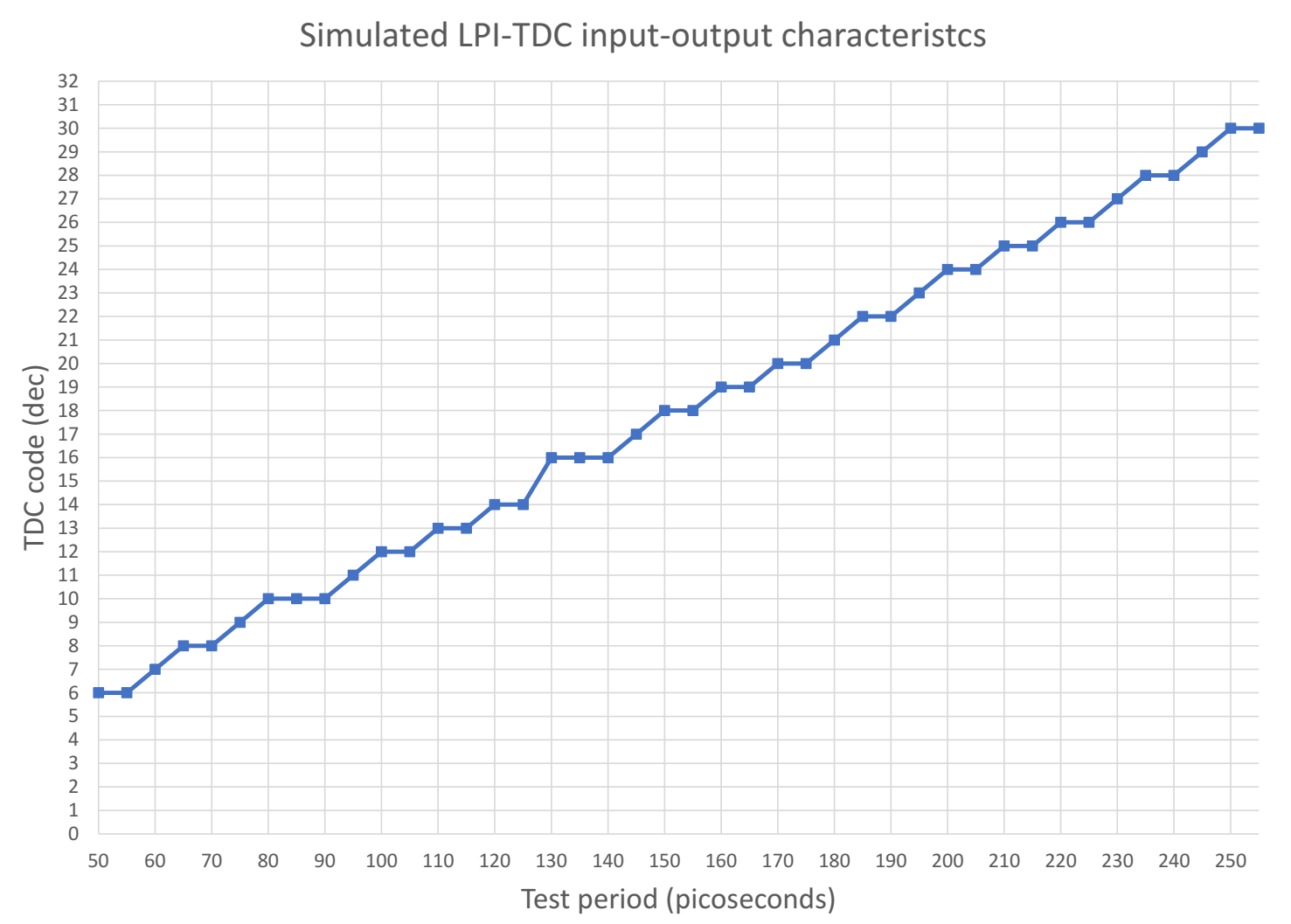

Figure 5.22: Simulated LPI-TDC output code behaviour in response to sweeping test period. Conditions: $\mathrm{f}_{\text {start }}=1 \mathrm{GHz}, \mathrm{f}_{\text {stop }}=100 \mathrm{MHz}, \mathrm{VDD}=1.4 \mathrm{~V}, \mathrm{~T}=65^{\circ} \mathrm{C}$.

\subsection{Summary of Simulation Results}

The design of an LPI-TDC has been presented in this chapter. The analysis has been divided into sub-blocks, such as the interpolation delay cell, sense amplifier, RS latch (that make up the SAFF), and the interface buffers. The individual block specifications have been derived based on the target LPI-TDC performance metrics. 


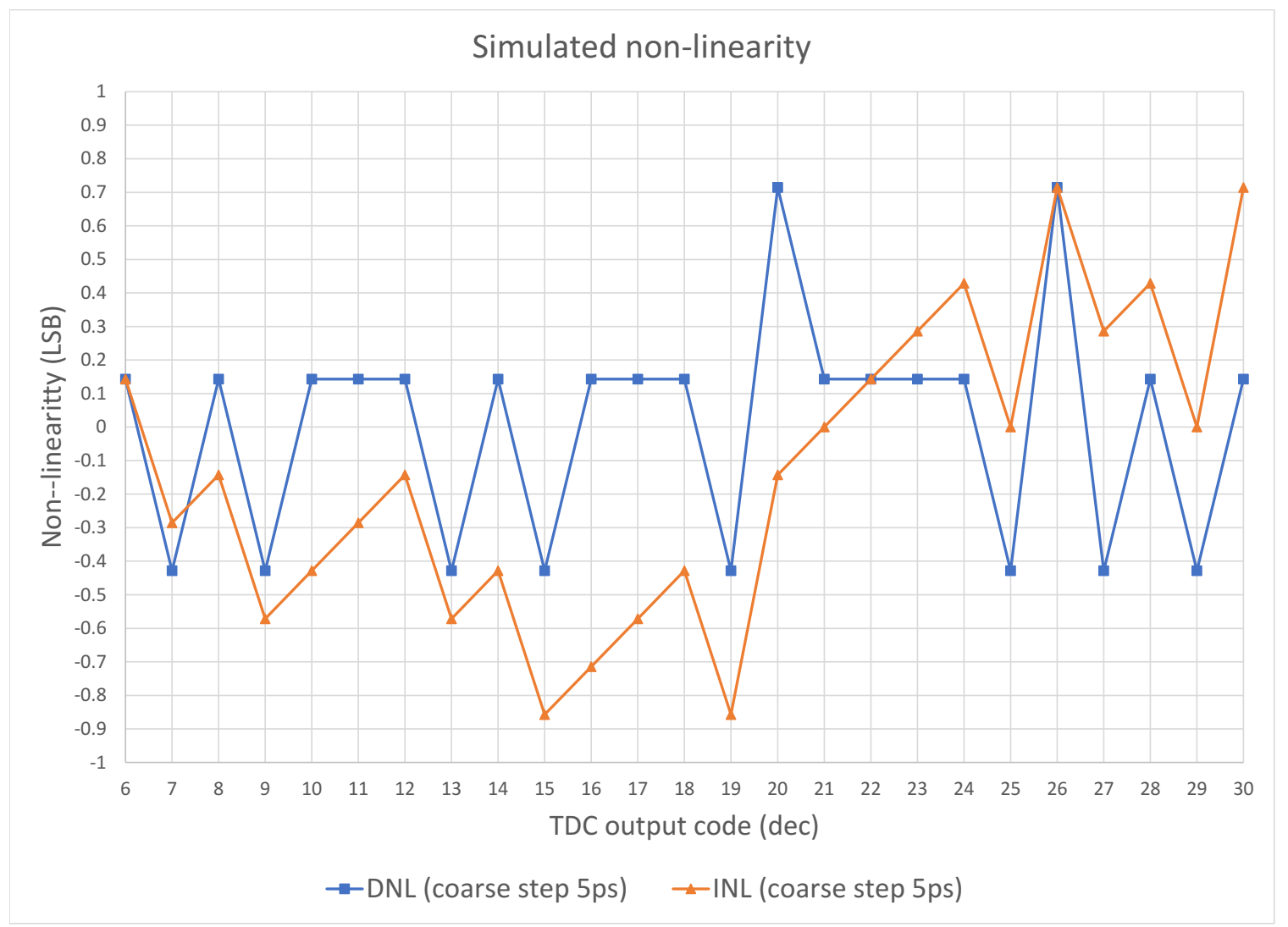

Figure 5.23: Simulated LPI-TDC non-linearity by sweeping test period. Conditions: $\mathrm{f}_{\text {start }}=1 \mathrm{GHz}, \mathrm{f}_{\text {stop }}=100 \mathrm{MHz}, \mathrm{VDD}=1.4 \mathrm{~V}, \mathrm{~T}=65^{\circ} \mathrm{C}$. 
A comprehensive summary of post-layout simulation results is given in Table 5.7 showing the feasibility of design to operate at the target specifications.

Table 5.7: List of the LPI-TDC performance metrics based on the extracted postlayout simulations.

\begin{tabular}{|c|c|c|c|c|}
\hline Parameter & units & Specification & Simulation 1 & Simulation 2 \\
\hline$f_{\text {start }}$ & $\mathrm{GHz}$ & 5 & 5 & 1 \\
\hline$f_{\text {stop }}$ & $\mathrm{MHz}$ & 100 & 100 & 100 \\
\hline Dynamic Range (fine TDC core) & ps & 255 & 258 & 273 \\
\hline $\mathrm{T}_{\mathrm{LSB}, \text { design }}$ & ps & 8 & 8.05 & 8.52 \\
\hline $\mathrm{SSP}_{\mathrm{LSB}}$ & LSB & $<1$ & $<0.5$ & $<0.5$ \\
\hline INL & LSB & $<1$ & $\mathrm{n} / \mathrm{a}$ & 0.86 \\
\hline DNL & LSB & $<0.5$ & $\mathrm{n} / \mathrm{a}$ & 0.43 \\
\hline VDD & V & 1.40 & 1.40 & 1.40 \\
\hline Temperature & ${ }^{\circ} \mathrm{C}$ & 65 & 65 & 65 \\
\hline $\begin{array}{l}\text { Power }\left(\propto \mathrm{f}_{\text {start }}\right) \\
\text { (core interpolator\&buffers) }\end{array}$ & $\mathrm{mW}$ & 94.5 & 128.3 & 39.5 \\
\hline $\begin{array}{l}\text { Power }\left(\propto \mathrm{f}_{\text {stop }}\right) \\
\text { (SAFF\& } \\
\text { Soutput buffers) }\end{array}$ & $\mathrm{mW}$ & 10.5 & 7.83 & 8.47 \\
\hline Power total (LPI-TDC ) & $\mathrm{mW}$ & 105.0 & 136.1 & 48 \\
\hline Area (core, buffer, SAFF) & $\mathrm{mm}^{2}$ & $<0.20$ & 0.09 & 0.09 \\
\hline $\mathrm{FOM}_{\mathrm{P}} \quad$ (fine TDC core) & Joules/bit & $4.77 \mathrm{p}$ & $5.65 \mathrm{p}$ & $6.76 \mathrm{p}$ \\
\hline Counter $(\mathrm{N})$ & count & 40 & 40 & 40 \\
\hline Power (counter |17|) & $\mathrm{mW}$ & $\approx 5$ & $\approx 5$ & $\approx 5$ \\
\hline Dynamic Range with counter & ns & 10.2 & 10.3 & 10.9 \\
\hline $\mathrm{FOM}_{\mathrm{P}} \quad($ coarse counter $[17]+$ fine TDC core $)$ & Joules/bit & $120.1 \mathrm{f}$ & $142.7 \mathrm{f}$ & $175.7 \mathrm{f}$ \\
\hline FOM $_{\mathrm{PAC}}$ (coarse counter $[17]+$ fine TDC core $)$ & $\$ \cdot$ Joules/bit & $48.1 \mathrm{p}$ & $25.7 \mathrm{p}$ & $31.6 \mathrm{p}$ \\
\hline
\end{tabular}




\section{Chapter 6}

\section{TDC Measured Results}

The TDC was manufactured at IBM foundry (Global Foundries) through the Canadian Microelectronics Corporation (CMC) in partnership with the MOSIS services. A total of 20 devices were ordered and bonded to a ceramic 44-pin flat-pack package CQFP44A, offered as part of the CMC post-fabrication service. The particular choice of assembly was selected from the available options for its optimal cost-to-performance trade-off. The close-up of the bare die attached to the package is presented in Figure 6.1

An on-chip characterization has been previously shown in other works 27]. However, such circuitry is unavailable in this design, which relies on the external equipment for parametric evaluation. To measure the device performance, a custom printed circuit board (PCB) was designed and manufactured to interface with the bench instrumentation. This chapter presents the measurement methodology, challenges, and parametric performance of this TDC. The test plan and configuration is discussed in Section 6.1. The PCB design is introduced in Section 6.3 with a detailed description followed in Appendix A. The evaluation of design offsets is presented in Section 6.4 The maximum frequency of operation is determined through tests discussed in Section 6.5. An additional investigation to determine the device performance limitations 


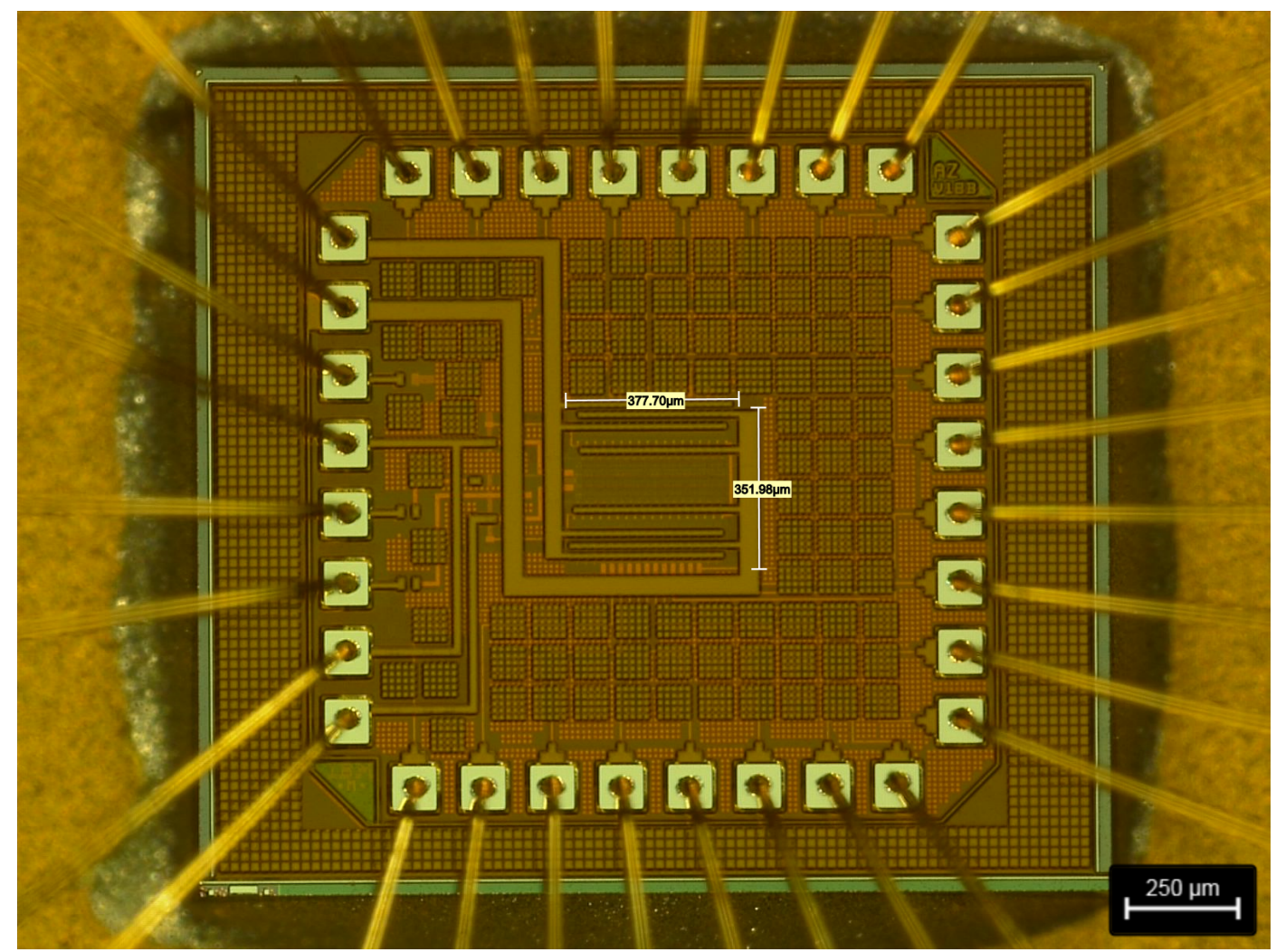

Figure 6.1: Photograph of the TDC bare die residing in the cavity of CQFP44A package. The bondwires attached to the die can be seen.

is presented in Section 6.6. A swept phase technique that was used to verify the dynamic performance metrics is described in Section 6.7 while the results of this measurement are further elaborated in Section 6.8 and Section 6.9 The final correlation between SSP and power consumption is discussed in Section 6.10 which shows a strong evidence of supply integrity (impedance) effect on the dynamic performance limits. The chapter is concluded with a complete summary and the interpretation of measured data in Section 6.11. 


\subsection{Design Evaluation Plan}

The key TDC metrics that need to be measured are single-shot precision (SSP), resolution $\left(\mathrm{T}_{\mathrm{LSB}}\right)$, and linearity (INL, DNL). The resolution and linearity are extracted from the phase sweep between start and stop (i.e. test period $\mathrm{T}_{\text {test }}$ ), while the SSP is determined from the TDC output code statistics. The following test methodology is applied:

1. set start signal frequency $\left(\mathrm{f}_{\text {start }}\right)$.

2. for each value of $\mathrm{f}_{\text {start }}$, sweep the $\mathrm{T}_{\text {test }}$ and sample multiple TDC output codes

The 24-bit wide output of TDC has to be captured and converted into a binary or decimal code, after optional bubble-error correction. A logic analyzer (Agilent 16903B frame with 16950A module) is used to probe and save the state of a 24-bit wide thermometer code at the $\mathrm{f}_{\text {stop }}$ rate.

The initial list of test conditions is summarized in Table 6.1.

Table 6.1: Measurements test matrix.

\begin{tabular}{lc}
\hline Parameter & Values \\
\hline VDD & $1.2 \mathrm{~V}, 1.4 \mathrm{~V}$ \\
$\mathrm{f}_{\text {start }}$ & $1 \mathrm{GHz}, 5 \mathrm{GHz}$ \\
$\mathrm{f}_{\text {stop }}$ & $100 \mathrm{MHz}$ \\
start, stop type & sin, square \\
$\mathrm{T}_{\text {test }}$ step & $5 \mathrm{ps}, 2 \mathrm{ps}$ \\
dynamic range & $500 \mathrm{ps}$ \\
number of devices & 5 \\
Temperature & $27^{\circ} \mathrm{C}$ (ambient) \\
\hline
\end{tabular}

There are a total of 14000 test points that have to be measured. An initial assessment of the test speed has shown that it would take approximately 5 minutes for 
each measurement in manual mode. This translates to almost 49 days of continuous non-stop measurements. To make the task more manageable, a 1 minute per test point is targeted. To make the measurements repeatable, ensure a safe power sequencing, minimize data collection errors, and to speed up the testing, it was decided to invest some time into test bench automation using MATLAB infrastructure (sequencing, data capture, post-processing). Custom equipment drivers were written to extend the built-in MATLAB capabilities. Run control scripts were implemented, achieving a test time of 2 minutes per measurement (see Appendix B).

\subsection{Test bench Setup}

From the list of available equipment (Table 6.2), the test bench architectures were derived for LPI-TDC characterization.

Table 6.2: List of available lab equipment and designated usage.

\begin{tabular}{|c|c|c|c|}
\hline Function & Manufacturer & Model & Usage \\
\hline Power supply & Agilent & E3646A & $\begin{array}{l}\text { dual-channel power supply. } \\
\text { Provides independent power to } \\
\text { two power domains VddInt and VddBuf. }\end{array}$ \\
\hline Pattern Generator & Agilent & $81134 \mathrm{~A}$ & $\begin{array}{l}\text { Variable frequency }(3.3 \mathrm{GHz} \text { max. }) \text { pattern generator } \\
\text { for high speed differential start signal. }\end{array}$ \\
\hline Oscilloscope & Tektronix & TDS684B & $\begin{array}{l}500 \mathrm{MHz} \text { real-time oscilloscope for debug, } \\
\text { timing adjustments, and general monitor functions }\end{array}$ \\
\hline Function Generator & Agilent & 81150 & $\begin{array}{l}\text { dual-channel differential } 100 \mathrm{MHz} \text { clock } \\
\text { (stop signal) source }\end{array}$ \\
\hline Logic Analyzer & Agilent & $16903 \mathrm{~B}$ & $\begin{array}{l}68 \text {-channel } 667 \mathrm{MHz} \text { logic state analyzer using } \\
\text { high-density high speed probes (good signal integrity) }\end{array}$ \\
\hline Logic Analyzer & Agilent & $16702 \mathrm{~B}$ & $\begin{array}{l}64 \text {-channel } 167 \mathrm{MHz} \text { logic state analyzer using } \\
\text { low-density probes (easier to debug) }\end{array}$ \\
\hline Vector Network Analyzer & Anritsu & $37347 \mathrm{C}$ & s-parameter measurement to analyze input matching \\
\hline Spectrum Analyzer & Agilent & N9320B & $\begin{array}{l}\text { spectrum analyzer to analyze signal quality } \\
\text { (phase noise) of start and stop signals }\end{array}$ \\
\hline
\end{tabular}

Two test bench configurations were used for bring-up and measurements, named Bench1 and Bench2. 


\subsubsection{Bench1 Description}

The Agilent 81150 is used as system reference clock generator. It generates the stop signal output, a $180^{\circ}$ sampling clock (CLK) for the logic analyzer, to capture a settled TDC output code. It is also used as a reference clock for the Agilent 81134 pattern generator which then uses an internal PLL to output a phase-locked start signal to the LPI-TDC device under test (DUT). This ensures that the phase relationship between the start and the stop signal remains static, thus creating a well-controlled phase difference between the two signals (i.e. $\mathrm{T}_{\text {test }}$ ), while allowing an independent phase adjustments between the two sources. To sweep the $T_{\text {test }}$, the Agilent 81150 output phase is adjusted.

The minimum phase step adjustment of the 81150 source is $0.1^{\circ}$, which at 100 $\mathrm{MHz}$ equates to $2.8 \mathrm{ps}$. This is sub-optimal for DNL and INL measurements, but sufficient for device characterization.

To complete the measurement, the logic analyzer (either 16903B or 16702B) frame, captures the LPI-TDC thermometer code that comes from the DUT over many cycles of the CLK signal (synchronous with the stop) for accurate SSP measurements. The resulting log file is then sent to a computer for post-processing in MATLAB.

The setup is controlled by a computer through standard communication protocols such as TCP/IP, GPIB, USB. This has also enabled precise power measurements thanks to the self-reported current and voltage sensing of the Agilent 3646 supply. The power measurements are observed over many cycles of the CLK signal, capturing the peak-to-peak and root-mean-squared (RMS) values for each data point. 


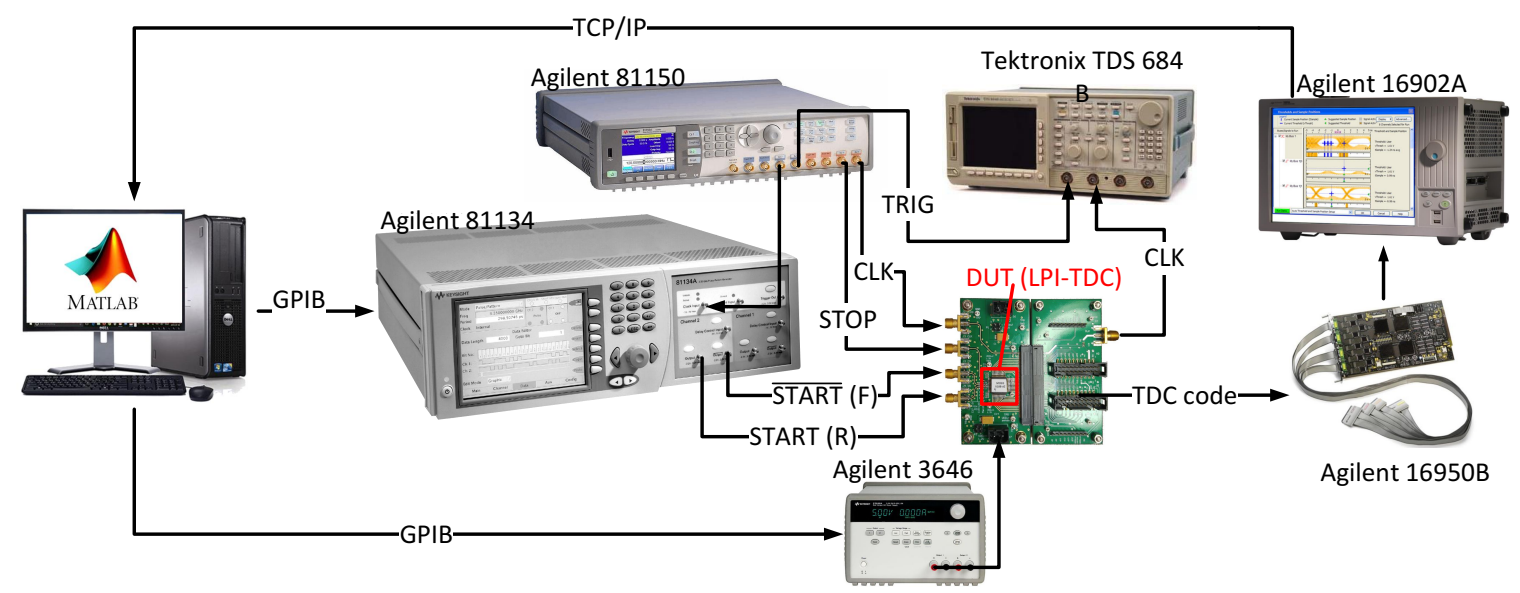

Figure 6.2: Bench v1 functional diagram.

\subsubsection{Bench2 Description}

The Bench1 setup suffers from a poor $\mathrm{T}_{\text {test }}$ control, because the finite loop bandwidth of the Agilent 81134 pattern generator causes a phase wander between the stop and start signals. This magnifies the measured SSP as it adds to the self-generated noise of the DUT (LPI-TDC ). Assuming the noise has a Gaussian distribution, the linearity and resolution should not be affected, by observing and averaging the TDC output code over many cycles.

To achieve better SSP measurements, the phase wander between the start and stop signal should be minimized (i.e. $\mathrm{T}_{\text {test }}$ appears more constant). This can be achieved, if both signals are derived from a single PLL synthesizer. Using the dual output of the Agilent 81134 achieves exactly this. However, the limited selection of divide ratios between the two outputs of 81134 source leaves a very limited range of frequencies that can be tested. Another disadvantage of using the Agilent 81134 is its poor implementation of the independent phase adjustment, making it difficult to automate the setup and requiring lengthy manual testing (about 3.5 minutes 


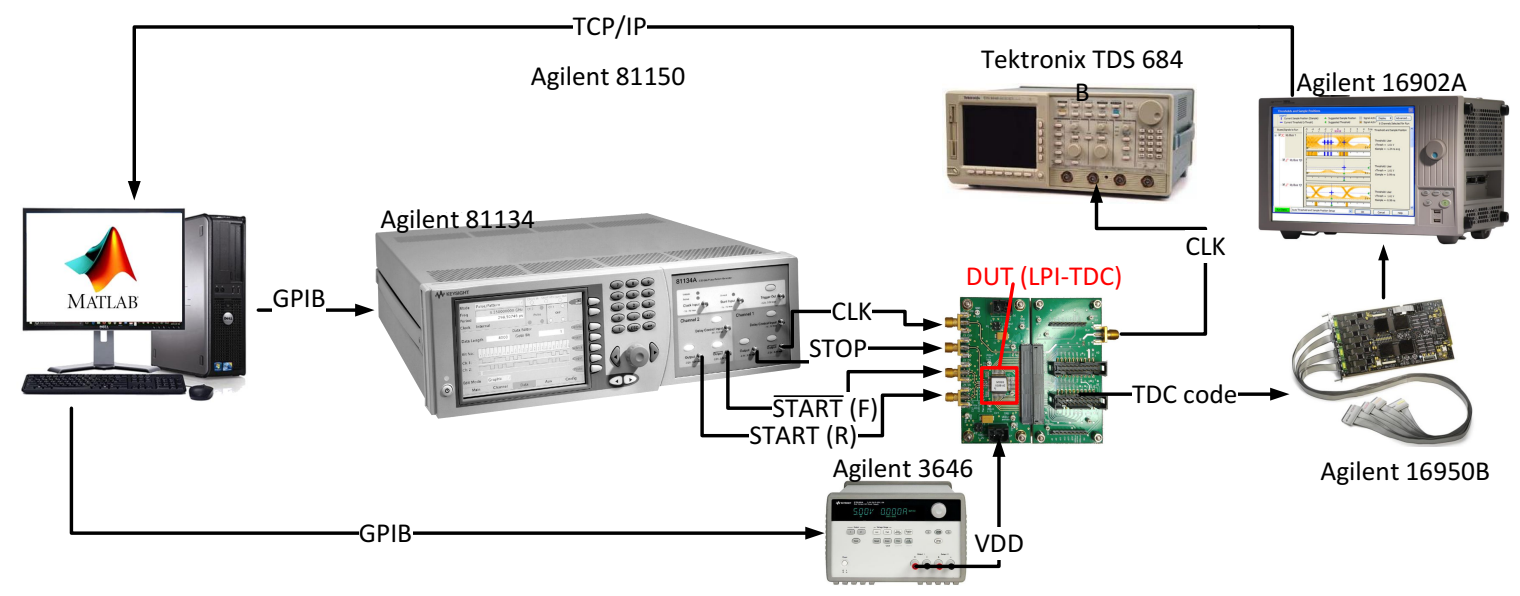

Figure 6.3: Bench v2 functional diagram

per single measurement point). However, the ability to de-embed the inherent LPITDC performance is much better compared to the Bench1 setup.

The rest of the Bench2 system operation is otherwise identical to the Bench1 (Section 6.2.1).

\subsubsection{System Noise Measurements}

The Bench1 setup can provide higher frequency for the start signal with a fine granularity compared to the Bench2 (Section 6.2.4). However, the Bench1 system noise is inherently higher. To confirm this, the Bench1 setup noise contribution to the $\mathrm{T}_{\text {test }}$ period is quantified.

The phase noise of the Agilent 81150 stop signal source was measured at $100 \mathrm{MHz}$ using the Agilent N9320B analyzer (Figure 6.4). Both sinusoidal and square clock shapes were analyzed. The phase noise of the Agilent 81134 start signal source was also measured at various frequencies $(1 \mathrm{GHz}, 1.7 \mathrm{GHz})$.

Using the post-processing scripts written in MATLAB, the source jitter in time domain was determined by integrating the measured phase noise power between 100 


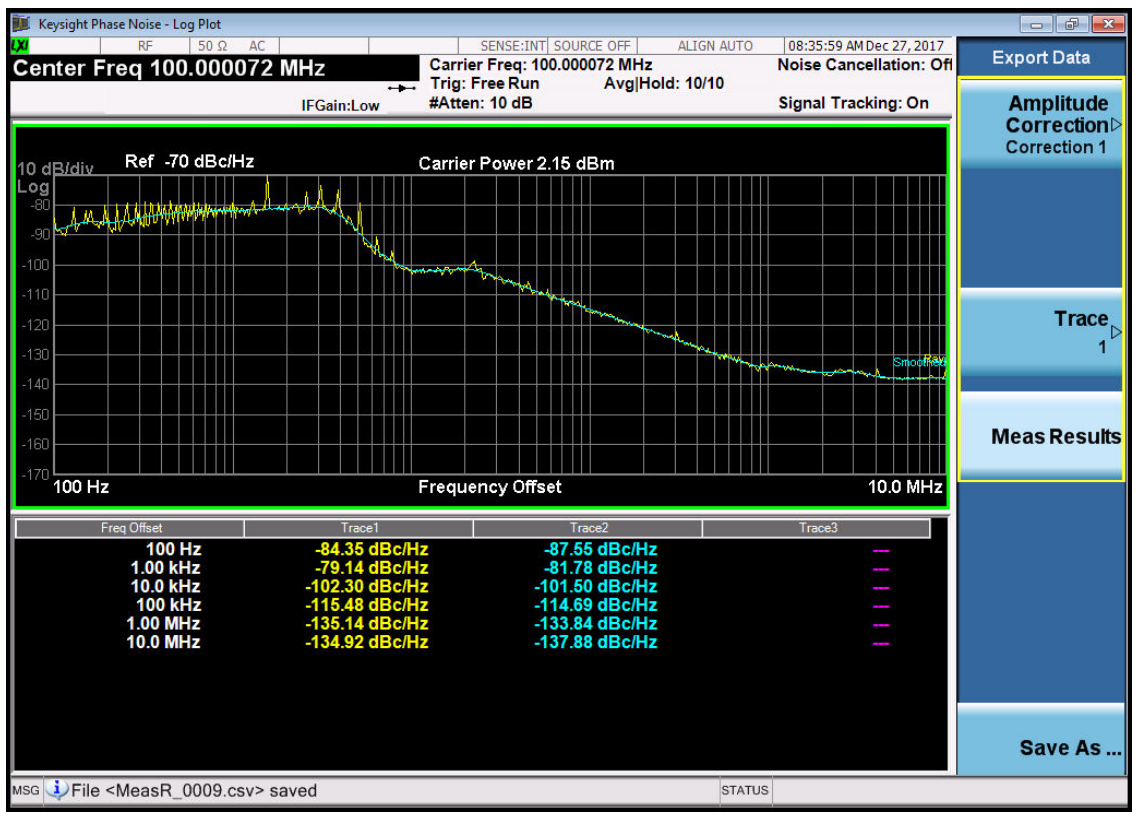

Figure 6.4: Phase noise of Agilent 81150 measured to quantify its impact on TDC performance

$\mathrm{Hz}$ and $\frac{f_{\text {carrier }}}{2}$ offset relative to the carrier. The lower integration boundary of 100 $\mathrm{Hz}$ was chosen based on the expected accumulation period of the Logic Analyzer, which has a finite state depth capture limit. The conversion from the phase noise to jitter is done using the same integration method as described in Section 3.1 Various output conditions and patterns were explored to determine the lowest noise mode for the TDC characterization.

The results are summarized in Table 6.3

Table 6.3: Equipment noise measurements for SSP characterization.

\begin{tabular}{lll}
\hline Source & frequency & RMS Jitter $\left(\mathrm{J}_{\mathrm{RMS}, \mathrm{src}}\right)$ \\
\hline 81150 & $100 \mathrm{MHz}$ & $15.4 \mathrm{ps}$ \\
81134 & $1 \mathrm{GHz}$ & $4.7 \mathrm{ps}$ \\
81134 & $1.7 \mathrm{GHz}$ & $4.7 \mathrm{ps}$ \\
\hline
\end{tabular}

It is not clear to what degree the output noise of 81134 and 81150 are going to 
be correlated. However, it is clear that the 81150 source will dominate the jitter in Bench1 setup, which will result in an undesired phase wander contribution to LPITDC on the order of $15 \mathrm{ps}\left(\sqrt{J_{R M S, 81150}^{2} \pm J_{R M S, 81134}^{2}} \approx 15 p s\right)$.

With the expected LPI-TDC resolution of 8ps, the Bench1 setup would degrade the SSP measurement by $\frac{15 p s}{8 p s} \approx 2 L S B$. This was confirmed in measurements when compared to Bench2 setup under the identical bias and input conditions Section 6.8.1

Therefore, to approximate the de-embedded LPI-TDC SSP value using Bench1 setup, it would require to subtract about 2 LSB from the measured value. However, a more accurate method would be to use the Bench2 setup, where the RMS noise of equipment is $<1 \mathrm{LSB}$ of the DUT resolution.

\subsubsection{Test Bench Summary}

The two test benches have slightly different purposes that complement each other to help de-embed the LPI-TDC performance. The pros and cons of both and the intended usage is outlined in Table 6.4

Table 6.4: Comparison of Bench1 and Bench2 setups and their preferred usage.

\begin{tabular}{|c|c|c|c|}
\hline Setup & Pros & Cons & Usage \\
\hline Bench1 & $\begin{array}{l}\text {-better automation (faster to run) } \\
\text { •wider selection of test frequencies }\end{array}$ & $\begin{array}{l}\text { - large } \mathrm{T}_{\text {test }} \text { phase wonder } \\
\text { •coarse } \mathrm{T}_{\text {test }} \text { step control }\end{array}$ & $\begin{array}{l}\text { - Maximum Frequency } \\
\text {-coarse INL, DNL } \\
\text {-coarse resolution }\end{array}$ \\
\hline Bench2 & •low $\mathrm{T}_{\text {test }}$ phase wonder & $\begin{array}{l}\bullet \text { poor automation (slower to run) } \\
\text { •small choice of usable frequencies }\end{array}$ & $\begin{array}{l}\bullet \text { SSP } \\
\bullet \text { fine INL, DNL } \\
\bullet \text { fine resolution }\end{array}$ \\
\hline
\end{tabular}

\subsection{Evaluation PCB Design}

In order to interface with the device a custom printed circuit board (PCB) was designed. A modular architecture was used to mitigate cost, risk, and complexity. The 
design was split into two parts: core and probe cards. The core card PCB contains the DUT along with input matching network, power distribution, on-board decoupling, and limited on-board test points for debugging. The probe card PCB contains the connectors for logic analyzer cables, redundant clock ports, test points, and additional hooks for the power supply distribution. The core and probe cards mate through a plug-in connector interface.

Two Agilent logic analyzers frames were available for measurements: 16702B (older) and 16903B (newer). The comparison is given in Table 6.5

Table 6.5: Logic analyzers available for the LPI-TDC characterization.

\begin{tabular}{lcc}
\hline Parameter & Agilent 16702B & Agilent 16903B \\
\hline Scope module & Agilent 16715A $(\times 2)$ & Agilent 16950A $(\times 1)$ \\
Channels (total) & 64 & 68 \\
Scope connector & 20-pin 0.1" pitch & 100-pin 0.5mm Samtec \\
State capture rate & $133 \mathrm{MHz}$ & $600 \mathrm{MHz}$ \\
Measurement automation & limited & comprehensive \\
\hline
\end{tabular}

The older 16702B logic analyzer uses a larger probe connector which would be easier to solder and use manually, but it comes at a cost of lower bandwidth and limited programming capabilities. The newer 16903B frame is much better for the signal integrity and automation, but the scope connector is quite challenging to solder and debug manually due to the fine pitch. Assessing the pros and cons of both modules, it was decided to enable the test using both systems, prioritizing the more powerful 16903B as the main solution and 16702B as the fall-back.

A total of 3 PCB card types (see Table 6.6) have been designed using the Altium Version 17 software. The intended usage of the two plug-in probe card variants is shown in Figure 6.5. 


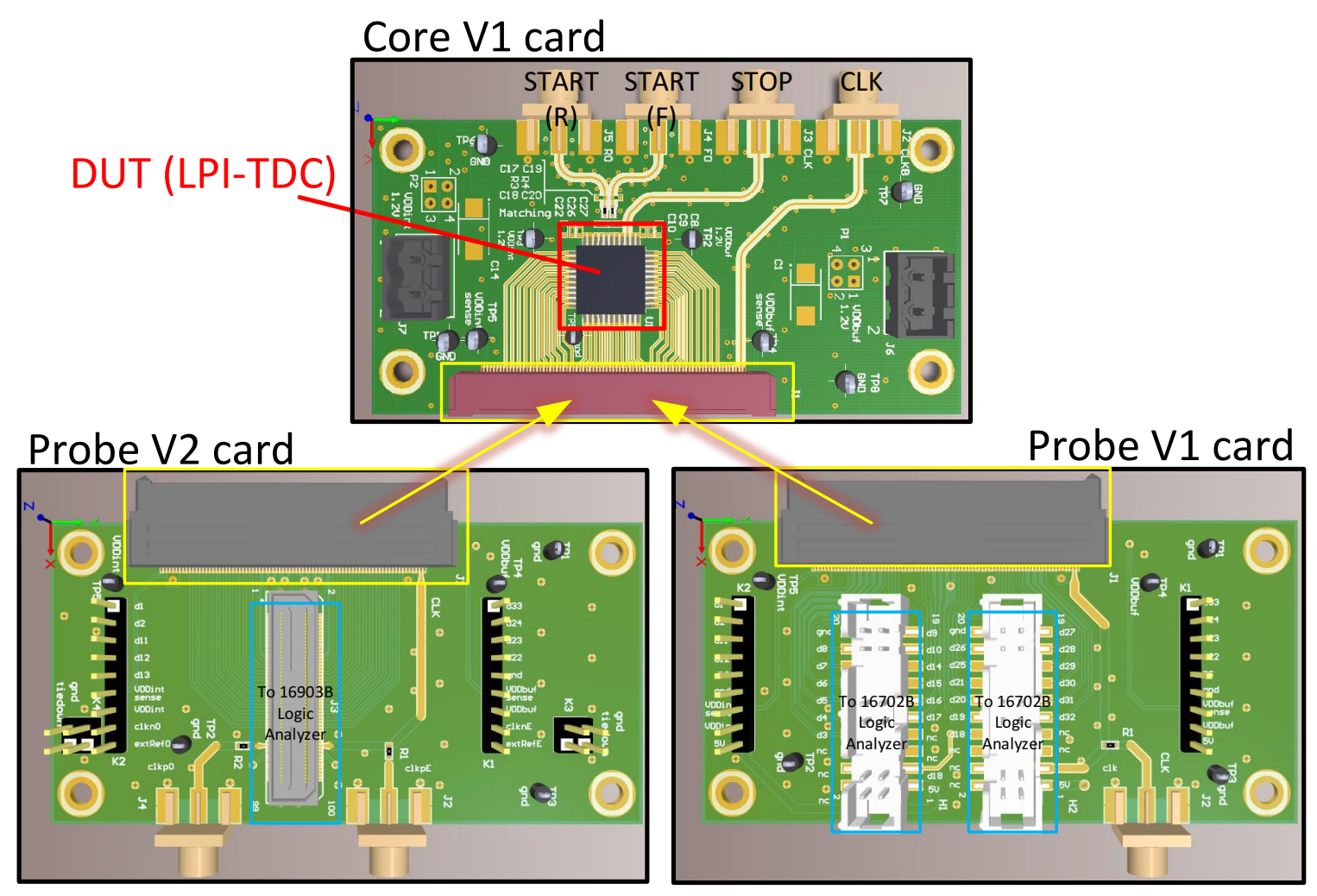

Figure 6.5: Modular use of test board variants allows for flexible test and debug solution

All boards were manufactured by the PCBway facility [28], as it provided sufficient process capabilities, cost, and design turn-around speed.

The board assembly was done by hand at Carleton University lab facilities, using custom-made solder re-flow ovens to help with the surface-mounted (SMT) components (courtesy of the lab coordinator Nagui Mikhail). The assembled boards with the proposed LPI-TDC were used to measure device characteristics (Figure 6.6).

Applying the test apparatus described in Section 6.1 the two test bench configurations were used to measure the maximum frequency of operation $\left(\mathrm{f}_{\text {start,max }}\right)$, Single-Shot Precision (SSP), Integral and Differential Non-linearity (INL, DNL), and power consumption. For a detailed discussion of the test board design challenges and 
Table 6.6: $\mathrm{PCB}$ variants

\begin{tabular}{|c|c|c|c|c|}
\hline PCB name & Description & Board type & $\begin{array}{l}\text { dimensions } \\
(\mathrm{mm} \times \mathrm{mm})\end{array}$ & $\begin{array}{l}\text { Number } \\
\text { of boards }\end{array}$ \\
\hline core card v1 & $\begin{array}{l}\text { DUT, high-speed routing, } \\
\text { power }\end{array}$ & $\begin{array}{l}\text { 4-layer, } \\
\text { FR-4, } \\
\text { ENIG finish }\end{array}$ & $50 \times 100$ & 5 \\
\hline probe card v1 & $\begin{array}{l}\text { interface to } 16702 \mathrm{~B} \text { frame } \\
\text { (16715 logic analyzer) }\end{array}$ & $\begin{array}{l}\text { 2-layer, } \\
\text { FR-4, } \\
\text { HASL finish }\end{array}$ & $50 \times 100$ & 1 \\
\hline probe card v2 & $\begin{array}{l}\text { interface to } 16903 \mathrm{~B} \text { frame } \\
\text { (16950 logic analyzer) }\end{array}$ & $\begin{array}{l}\text { 2-layer, } \\
\text { FR-4, } \\
\text { HASL finish }\end{array}$ & $50 \times 100$ & 2 \\
\hline
\end{tabular}

solutions please see Appendix A.3

\subsection{Initial Bring-up and Device Offsets}

For a robust operation of the proposed LPI-TDC it is required that the differential input signal start maintains symmetry switching point around $\frac{V D D}{2}$ as it propagates through the interpolation core. The positive side (rising edge) is labelled $R$ or START $(R)$ in Figure 6.6. By analogy, the negative side (falling edge) is labelled F or START $(F)$.

By design, the LPI-TDC stops functioning if both sides of the start input are held at the same potential. A large static DC current flow is developed across the interpolation core delay cells. The strength of this common-mode current is limited by the impedance of interpolation resistors and MOSFET devices within the delay cells. The current flow stops when the inputs return to differential operation (i.e. commonmode offset is equal to full rail-to-rail swing). The voltage level when this transition occurs reveals the switching point of the inverters inside the LPI-TDC delay unit cells. This mode of operation is exploited to test the on-chip circuit functionality and quantify the input-referred offsets by observing the power consumption as a function of input common-mode offset potential. The $\mathrm{R}$ and $\mathrm{F}$ of start signal are tested 


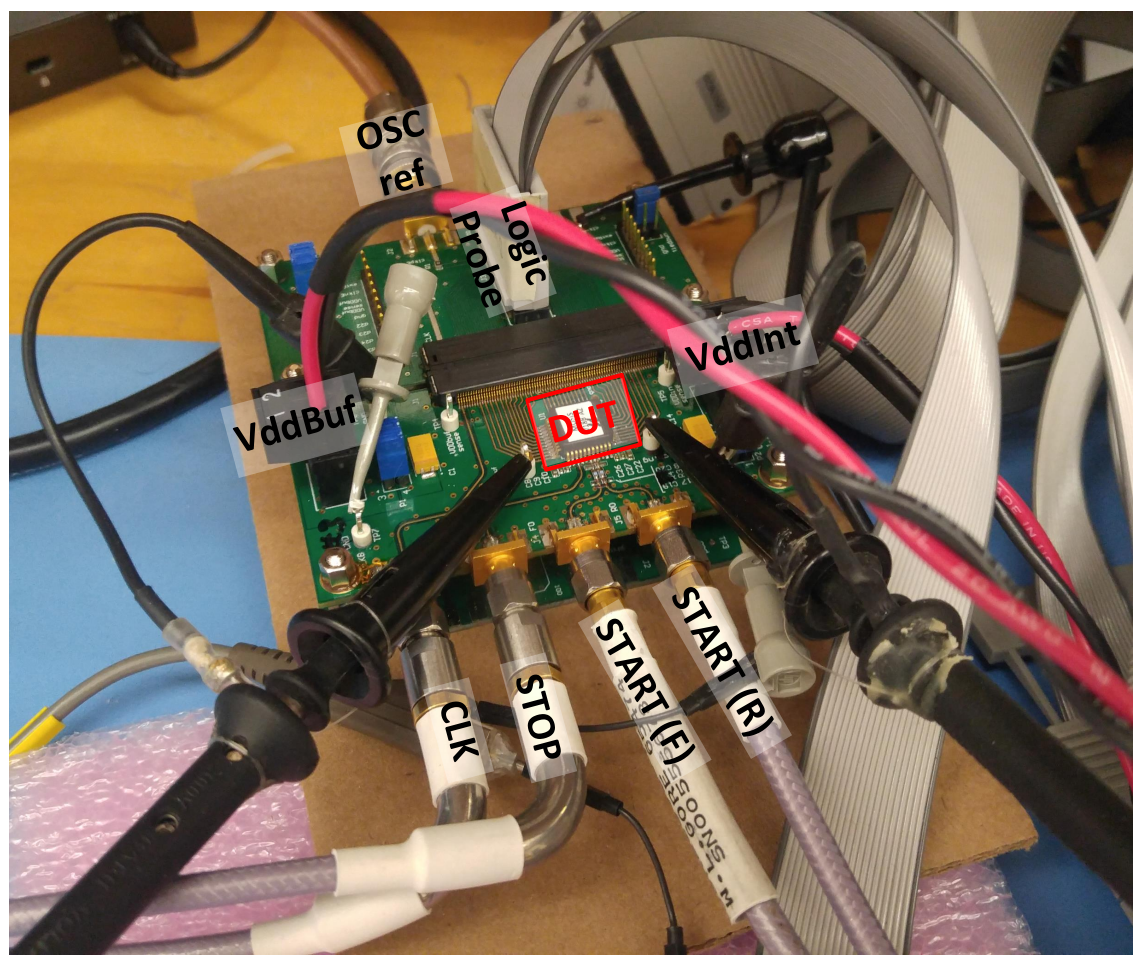

Figure 6.6: Assembled Core and Probe (v2) cards used in measurements.

independently to understand the mismatches within interpolation core.

The following method was used to determine the switching offsets:

1. Both inputs are held at logic "low" 0V potential to form static common-mode current.

2. Gradually increase the voltage on either $\mathrm{R}$ or $\mathrm{F}$ sides (i.e. common mode offset).

The power consumption on VddInt domain will reduce dramatically once the interpolation core returns to differential (complimentary) mode of operation, limited only by MOSFET device leakages.

3. repeat the procedure for both sides of differential start signal.

The stop signal (clock) was held at $\frac{V D D}{2}$ to keep the SAFF latches in transparent state, thus causing some change in current consumption on the VddBuf rail when the interpolation core returns to the differential mode of operation. 


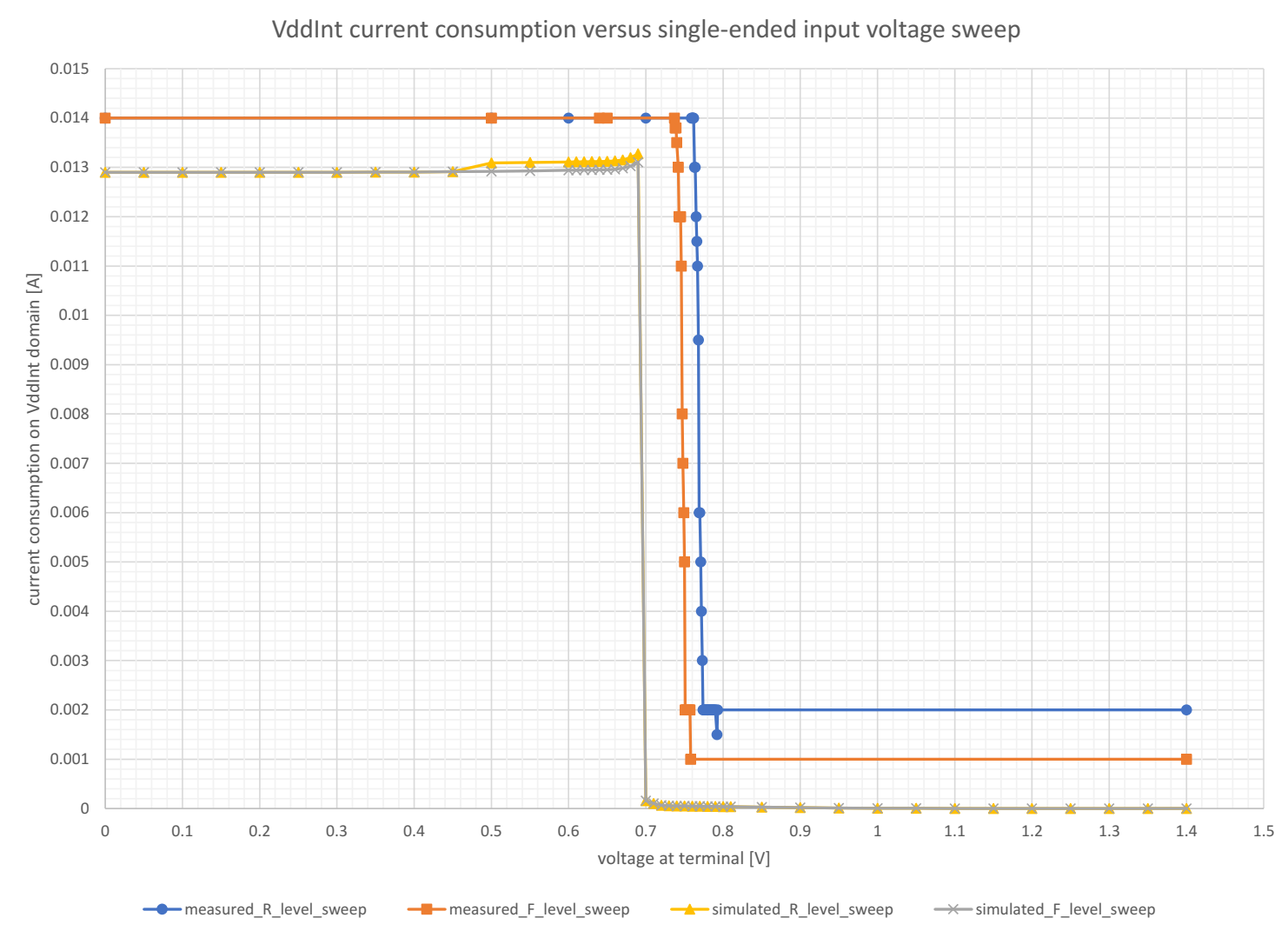

Figure 6.7: Measured and simulated current consumption as a function of singleended input level voltage.

This methodology was verified in simulations to correlate with the measurements (Figure 6.7).

The absolute power is limited by the device offsets and mismatches. The measured current consumption appears to match the simulated results, when a $10 \mathrm{mV}$ offset is added at the interpolator core input. This is just an approximation, but it suggests that the internal offsets are relatively small. A systematic shift (seen on horizontal axis $)$ of $\approx 48 \mathrm{mV}(\approx 3.5 \%)$ relative to the simulated result reveals an imbalance between the PFET and NFET (pull-up and pull-down) strengths. Moreover, the F and $\mathrm{R}$ curves switch at slightly different input level, measuring a delta of $\approx 20 \mathrm{mV}$. This is most likely explained by the input buffer mismatches between the two sides 
of the complimentary input.

The overall magnitude of design offsets is relatively small and should leave a healthy margin for operation (relative to rail-to-rail switching amplitudes), especially at the lower frequencies of operation. Further improvements are possible from a careful optimization of the $\mathrm{R}$ and $\mathrm{F}$ sides of the start input signal during the measurement phase by applying the optimal swing at each port.

\subsection{Maximum Frequency of Operation}

Using Bench1 setup (Section 6.2.1), the maximum frequency of operation was determined to be $\geq 1.7 \mathrm{GHz}$. Attempting to increase the $\mathrm{f}_{\text {start }}$ frequency beyond this point stops the LPI-TDC from generating the expected thermometer code. This also correlates with an abrupt drop in power consumption on both VddInt and VddBuf power domains, suggesting that the high speed input signal does not even reach the interpolator core. The assembly faults were ruled out since all core cards with different DUT and matching networks have shown similar behaviour, varying by $\approx 200$ $\mathrm{MHz}$ between the best and the worst performers.

The input matching of stop signal was examined more closely. The simulation results in Section A.1.1 indicated that the lack of the on-chip termination could cause a significant signal integrity degradation without the PCB input matching network. An investigation was performed to understand the discrepancy between simulated and measured results. 


\subsection{Correlating Simulation and Measured Results}

Given the uncertainty of modelling accuracy in simulations (Section A.1.1, it was decided to experimentally determine which matching network performs best within the constraints of a chosen 3-element structure. A small range of RF-grade capacitors was ordered with values ranging between $1.5 \mathrm{pF}$ and $18 \mathrm{pF}$.

A total of five structures were measured using the Anritsu 37347C Vector Network Analyzer for analysis.

Table 6.7: A list of input matching experiments.

\begin{tabular}{lll}
\hline Board Index & Matching Network Topology & Measured File Name \\
\hline 2 & $\begin{array}{l}\text { series } \mathrm{R}=50 \Omega \\
\text { shunt } \mathrm{C}=2.7 \mathrm{pF}\end{array}$ & EB2_ID4_biasNA_S11 \\
\hline 5 & $\begin{array}{l}\text { series } \mathrm{R}=50 \Omega \\
\text { shunt } \mathrm{C}=8.2 \mathrm{pF}\end{array}$ & EB5_ID3_biasNA_S11 \\
\hline 3 & $\begin{array}{l}\text { series } \mathrm{R}=50 \Omega \\
\text { shunt } \mathrm{C}=2.7 \mathrm{pF}\end{array}$ & EB3_ID11_biasNA_S11 \\
\hline 4.2 & $\begin{array}{l}\text { series } \mathrm{C}=8.2 \mathrm{pF} \\
\text { shunt } \mathrm{R}=50 \Omega\end{array}$ & EB4p2_ID12_biasNA_S11 \\
\hline
\end{tabular}

Boards 2 and 3 were configured the same to eliminate the assembly error concerns. The input return loss (S11) magnitude is plotted in Figure 6.8

The conclusion is that none of the configurations yield $|\mathrm{S} 11|<-10 \mathrm{~dB}$, implying a generally poor match at frequencies of interest. Based on the S11 plots it is expected that measurements will be significantly impaired for start signal frequencies $\mathrm{f}_{\text {start }}>2$ GHz. For the purposes of characterization it was decided to keep the default (theoretical) input matching topology (series $\mathrm{R}=50 \Omega$, shunt $\mathrm{C}=2.7 \mathrm{pF}$ ), which at the very least provides the correct input biasing.

To confirm that the upper frequency limitation is mainly impaired by the signal integrity of DUT interfaces, the transient simulations were re-run once again. All 


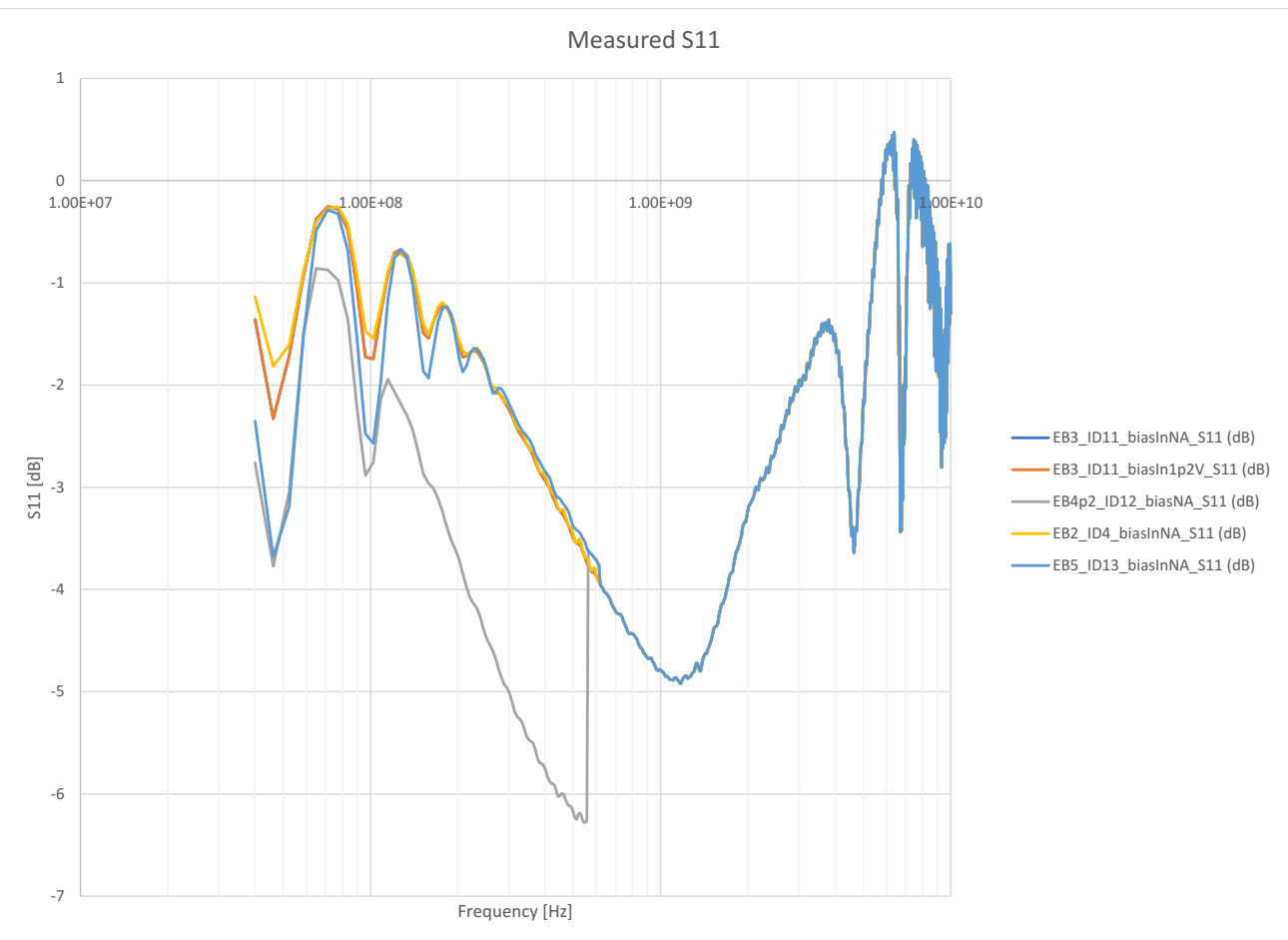

Figure 6.8: Comparison of various board matching techniques using S11 to identify the best option for measurements.

interface models were updated to include the supply and ground bond-wire effects as well as the internal device mismatch offsets (Section 6.4). With the updated models, the simulation results match measurements much closer, with the simulated upper limit reaching $f_{\text {start }} \approx 2.7 \mathrm{GHz}$. This degradation in simulated results was caused by primarily including a more accurate interconnect model around the DUT.

With more effort, it is possible to determine which additional modelling aspects caused the inaccuracy in simulated results. However, it doesn't change the fact that the lack of an on-chip termination is one of the main design limitations.

It is also possible that the $\mathrm{PCB}$ trace characteristic impedance is not a perfect 50 
$\Omega$ either which could further narrow the gap between simulated and measured results.

For completion, the clock path for the stop signal was also measured and has shown $\mathrm{S} 11 \approx-15 \mathrm{~dB}$ at $100 \mathrm{MHz}$, which should provide sufficient performance.

\subsubsection{Investigation Summary}

The upper frequency of the proposed LPI-TDC (ICGCUKIK) is mainly caused by the signal integrity issues at the DUT input. Using a more accurate interconnect model around the DUT helped narrow the gap between measured $(1.7 \mathrm{GHz})$ and simulated $(\approx 2.7 \mathrm{GHz})$ results. The lack of an on-chip termination is one of the main design limitations. With more careful attention to signal integrity, it would be possible to achieve much higher frequency of operation.

The dynamic performance metrics are measured and reported up to the maximum frequency of operation $1.7 \mathrm{GHz}$. Experimentally it was also determined that the input logic "1" (high) level has to be backed off to VDD-50mV to achieve the maximum rate of operation. This could be the result of either device offsets or signal reflections that may cause the input swing to trip the on-chip ESD diodes thus deteriorating the quality of the internal supply.

\subsection{Swept Phase Measurement Technique}

With an expected resolution of 8 ps, only a window of about $192 \mathrm{ps}$ is available for observation. By itself it is not enough to see the full period of the start signal, given the frequency limitation of the DUT.

A swept phase technique is used to circumvent this limitation. The phase of the start signal is swept relative to the static stop phase. This is equivalent to 


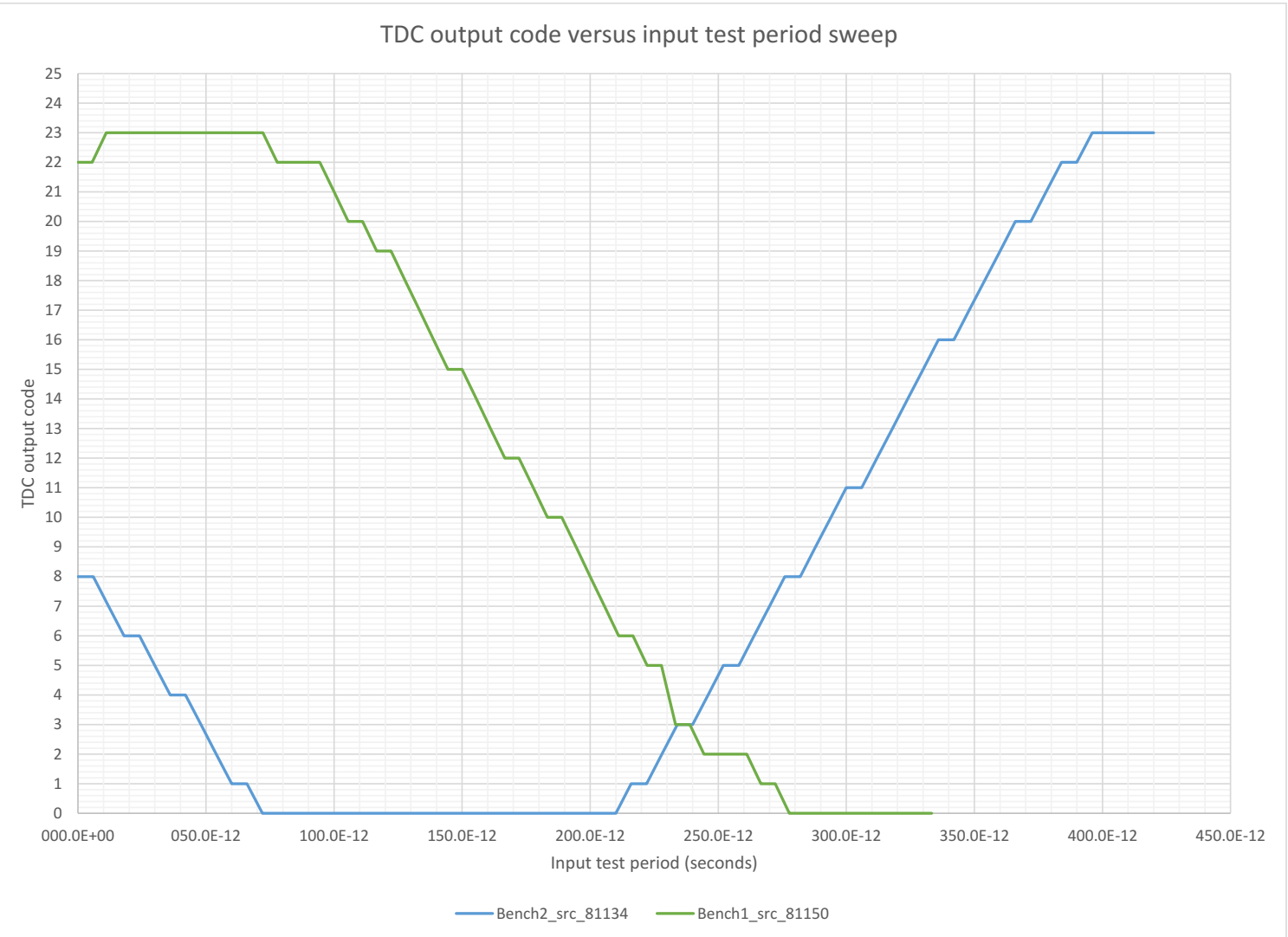

Figure 6.9: Demonstrating swept phase technique in action to observe LPITDC code transitions for further parametric characterization.

slowly sliding the phase of the high speed signal relative to the stop edge. When the start rising or falling edge phase lines up with the rising edge of the stop signal within a 192ps window, the code transitions can be observed at the output of LPI-TDC. Depending on which start edge is present in the LPI-TDC window of observation, the output value either increases or decreases. This technique enables the characterization of device non-linearity and resolution without the need to fit the full start period within the dynamic range of the LPI-TDC. When the start edge moves far away from the stop rising edge, the output code saturates to either all-0s or all-1s (decimal code 0 and 24 respectively). Internally the signal continues to toggle the interpolator core at the $\mathrm{f}_{\text {start }}$ rate, just as the stop continues to sample the saturated code. 
Linearly sweeping the input signal phase helps identify both falling and rising edges of the start input. The region of the observed code transition is subsequently used to characterize the dynamic performance of the proposed LPI-TDC.

This technique is demonstrated in Figure 6.9 showing the observed code transitions at $\mathrm{f}_{\text {start }}=1.6 \mathrm{GH} \mathrm{T}^{7}$ and $\mathrm{f}_{\text {stop }}=100 \mathrm{MHz}$ using Bench1 and Bench2 setup. The board number 3 (device ID 12) was biased at $\mathrm{VDD}=\mathrm{VddInt}=\mathrm{VddBuf}=1.4 \mathrm{~V}$. The horizontal axis shows the absolute phase difference (in seconds) of the start signal relative to stop, swept in 6ps steps. Unfortunately, the starting phase difference is almost always different when the equipment is reset between the experiments. This causes some measurements to start closer to the falling edge of the start signal, while others begin near the rising edge. This explains the difference in Bench1 and Bench2 apparent slopes. However, the dynamic performance metrics don't change and either edge can be used for comparison.

The start signal phase was swept manually due to the limited Bench2 automation capabilities. Collecting power and code statistics measurements at each data point takes almost 2.5 minutes. Traversing the $200 \mathrm{ps}$ (single edge) takes $>4$ hours under just one bias condition. Therefore, only a limited set of data was obtained using Bench2 setup. However, using Bench1 setup and a coarse phase step, the data was validated to stay consistent between the devices (i.e. part-to-part variation).

\subsection{SSP Measurements}

The single-shot precision was collected over a range of input conditions. Using an Agilent 16903B frame with a 16950 logic analyzer the TDC output thermometer code

\footnotetext{
${ }^{1}$ Although the upper limit of LPI-TDC is $f_{\text {start }} \geq 1.7 \mathrm{GHz}$, the closest output frequency available in Bench2 setup is $1.6 \mathrm{GHz}$ without going over.
} 
was captured over a course of $2.56 \mathrm{~ms}$ (a total of 256000 samples at a $100 \mathrm{MHz}$ sampling rate). The thermometer code was then converted to a decimal representation. Since only 24 physical lines (bits $<6>$ through $<29>$ ) are available for observation, the code was normalized to simplify the calculations, plotting and post processing. Therefore, the expected minimum code is 0 , while the maximum is 24 . The peak-topeak variation, standard deviation, and average code are extracted.

\subsubsection{Equipment Noise in SSP Measurements}

The SSP metric is only meaningful when the code transitions within the dynamic range of TDC, when device noise can dynamically influence the outcome of conversion. Once the interpolator core is saturated, the device noise has no perceived impact on the output code.

Using Bench2 setup and a coarse phase step size of $6 \mathrm{ps}$, the $\mathrm{SSP} \approx 1.5 \mathrm{LSB}$ is observed within the rising code transition region (phases 200 ps to $400 \mathrm{ps}$ ). Under the same conditions the Bench1 reports the SSP metric closer to 3.5 LSB (phases 70 ps to 280 ps) as seen in Figure 6.10 The delta of (3.5 LSB - 1.5 LSB)=2 LSB matches almost exactly the expected difference based on the system noise measurements in Section 6.2.3.

A quick observation also shows that the transition region measures 190-200 ps between the saturated code values. This indicates that the LPI-TDC interpolator

core resolution is $\approx \frac{195 p s}{(24-0)}=8.125 \mathrm{ps}$. However, the test phase step size of $6 \mathrm{ps}$ is too coarse to accurately determine the resolution. A more accurate value is extracted from a dedicated experiment in Section 6.8.2. 


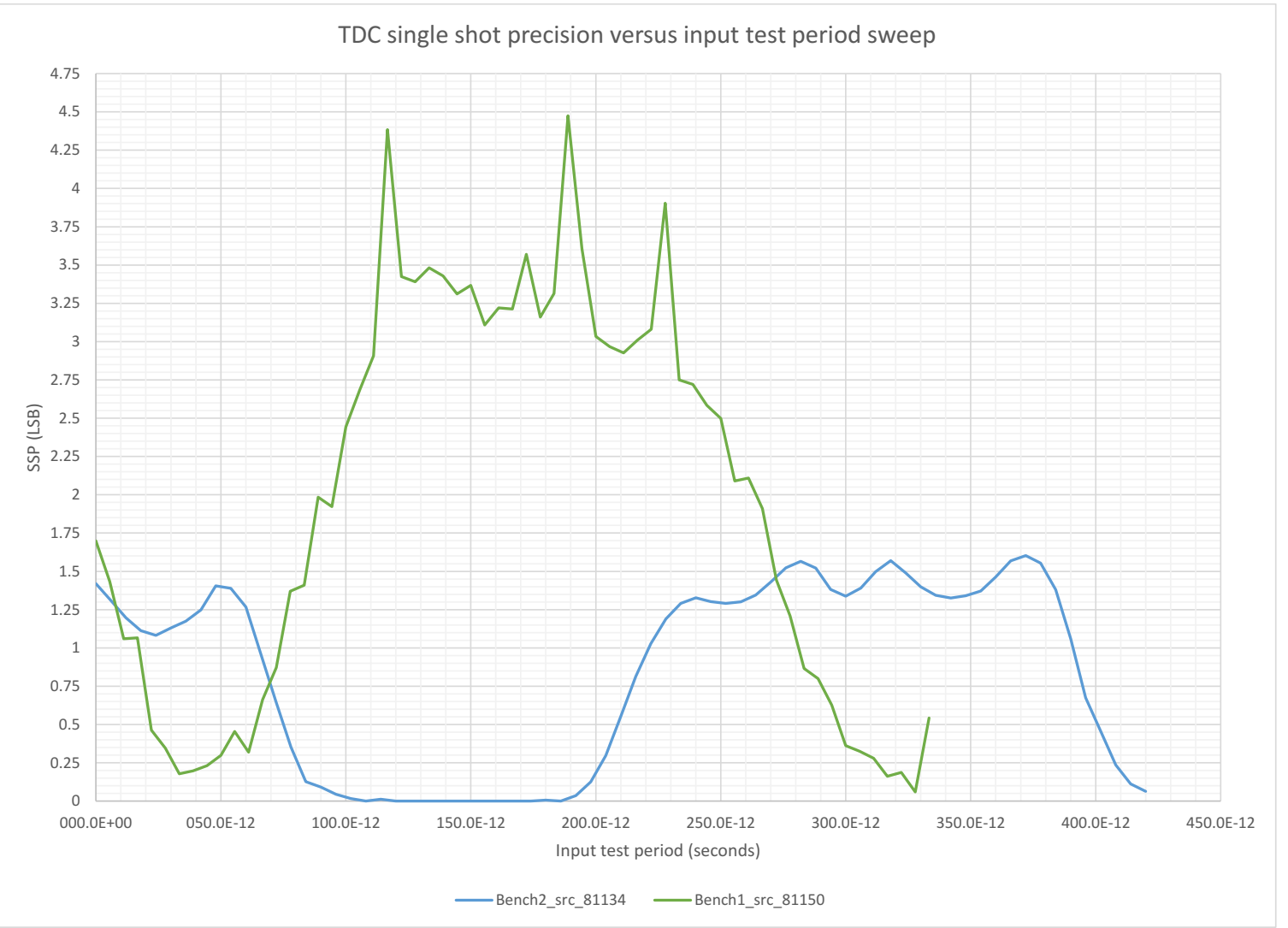

Figure 6.10: Demonstrating swept phase technique to assess SSP performance between Bench1 and Bench2 setups.

\subsubsection{Accurate SSP Measurement Summary}

Using Bench2 setup the start phase was swept with a 2 ps step size data point. For each phase the LPI-TDC output code was accumulated for $2.56 \mathrm{~ms}$ at $100 \mathrm{MHz}$ sampling rate. The code statistics (standard deviation $(\sigma=\mathrm{SSP})$ and the average value) were extracted at each step. Several operating conditions were examined and plotted in Figure 6.11. The LPI-TDC quantized decimal output is plotted in yellow stair waveform. The average code with $\operatorname{SSP}(\sigma)$ error bars are shown in red.

At $1.6 \mathrm{GHz}$ the $\mathrm{SSP}$ is $1.6 \mathrm{LSB}$, which drops to $0.9 \mathrm{LSB}$ when the start frequency is reduced to $0.8 \mathrm{GHz}$. This suggests that the observed SSP degradation is mainly due to the LPI-TDC device noise contribution. 

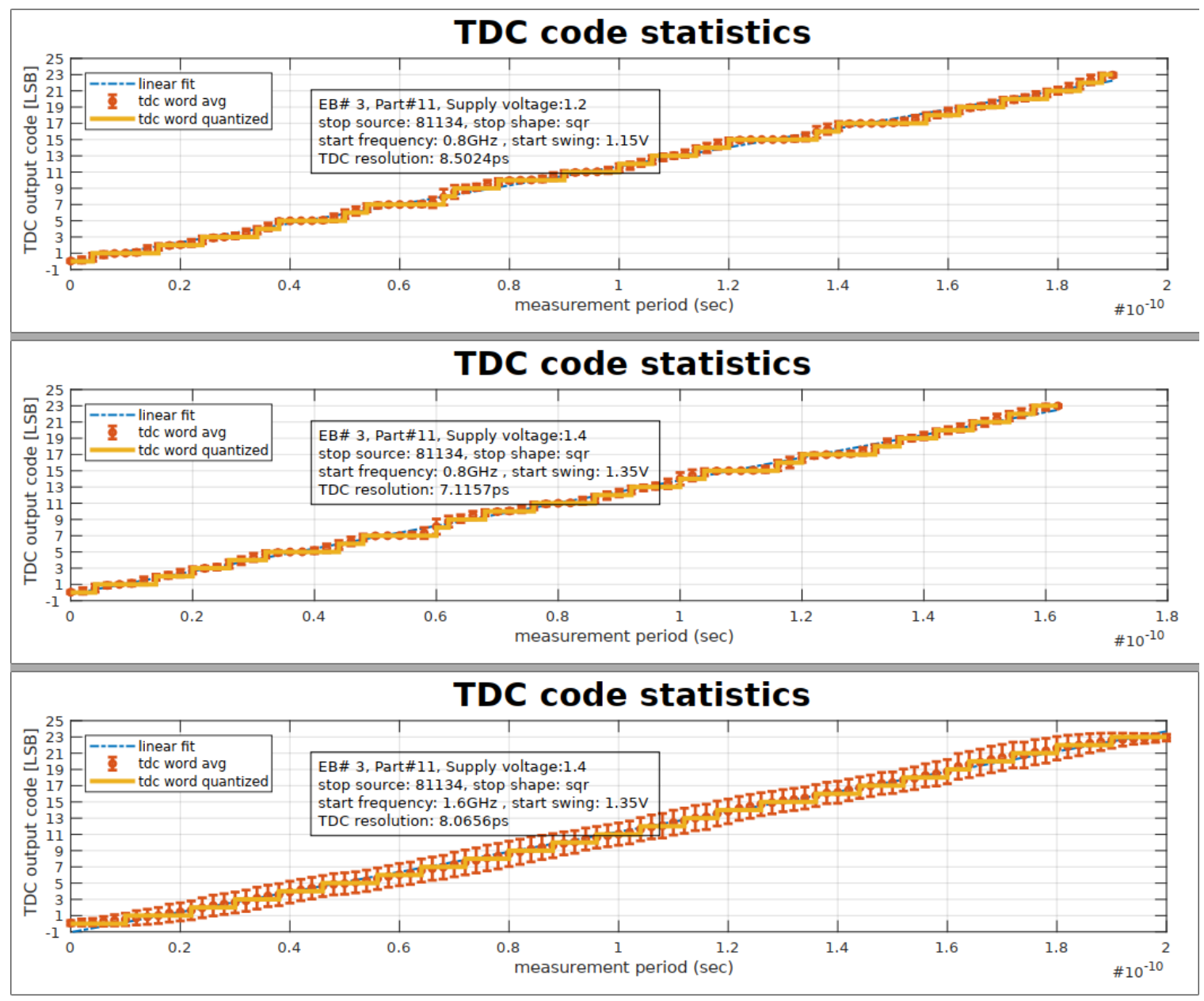

Figure 6.11: Measured code statistics of core card number 3 under various conditions: $\mathrm{VDD}=(1.4 \mathrm{~V}, 1.2 \mathrm{~V}), \mathrm{f}_{\text {start }}=(1.6 \mathrm{GHz}, 0.8 \mathrm{GHz}), \mathrm{f}_{\text {stop }}=100 \mathrm{MHz}$.

The most likely root cause is the sub-optimal on-chip power supply integrity, which generates a lot of noise during the sampling by the on-chip SAFF latches. The evidence for this was experimentally determined from the power measurements which strongly correlate with the SSP fluctuations in Section 6.10 (also observed in simulation). A closer inspection has revealed that the isolation buffers between the interpolation core and the SAFF chain were all assigned to the VddBuff domain, thus enabling the SAFF noise to directly couple into the core. A better supply-crossing buffer design and an improved supply decoupling would greatly reduce the SSP. 


\subsection{INL, DNL, and Resolution}

The non-linearity was extracted from the same data set that was used to obtain the SSP measurements in Section 6.8.2 The LPI-TDC INL and DNL were measured using the techniques discussed in Section 4.1.3 The line of best fit method was used for the INL and the code density test was applied to estimate the DNL with the highest degree of accuracy.

The same line of best fit was used to extract the converter slope $\left(\frac{p s}{L S B}\right)$, which represents the effective resolution of the proposed LPI-TDC. Without the trimming and compensation circuitry, the slope (resolution) changes with varying process corners, supply voltages, and temperature (PVT).

A typical measurement is presented in Figure 6.12, with the maximum values labelled in the plot.

The INL, DNL, and resolution measurements are summarized in Table 6.8

Table 6.8: Summary of LPI-TDC measured resolution and non-linearity.

\begin{tabular}{ccccccc}
\hline Board Index & $\begin{array}{c}\text { VDD } \\
(\mathrm{V})\end{array}$ & $\begin{array}{c}\mathrm{f}_{\text {start }} \\
(\mathrm{GHz})\end{array}$ & $\begin{array}{c}\mathrm{f}_{\text {stop }} \\
(\mathrm{GHz})\end{array}$ & $\begin{array}{c}\mathrm{INL}_{\mathrm{MAx}} \\
(\mathrm{LSB})\end{array}$ & $\begin{array}{c}\mathrm{DNL}_{\mathrm{MAx}} \\
(\mathrm{LSB})\end{array}$ & $\begin{array}{c}\text { resolution } \\
\left(\frac{p s}{L S B}\right)\end{array}$ \\
\hline 3 & 1.4 & $1.6 \mathrm{GHz}$ & $100 \mathrm{MHz}$ & 1.05 & 0.52 & 8.07 \\
\hline 3 & 1.4 & $0.8 \mathrm{GHz}$ & $100 \mathrm{MHz}$ & 1.06 & 0.76 & 7.12 \\
\hline 3 & 1.2 & $0.8 \mathrm{GHz}$ & $100 \mathrm{MHz}$ & 1.03 & 1.0 & 8.50 \\
\hline
\end{tabular}

More data was acquired from Bench1 to check 4 other Core Cards builds (i.e. devices). The results confirmed that all boards behave almost identically, which makes the data presented above representative of the proposed design. 
TDC code statistics

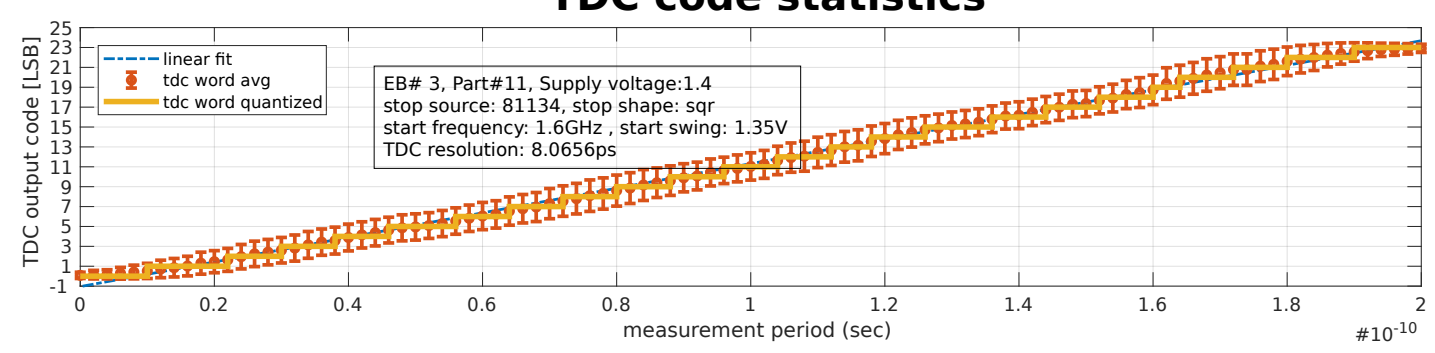

INL vs measurement period

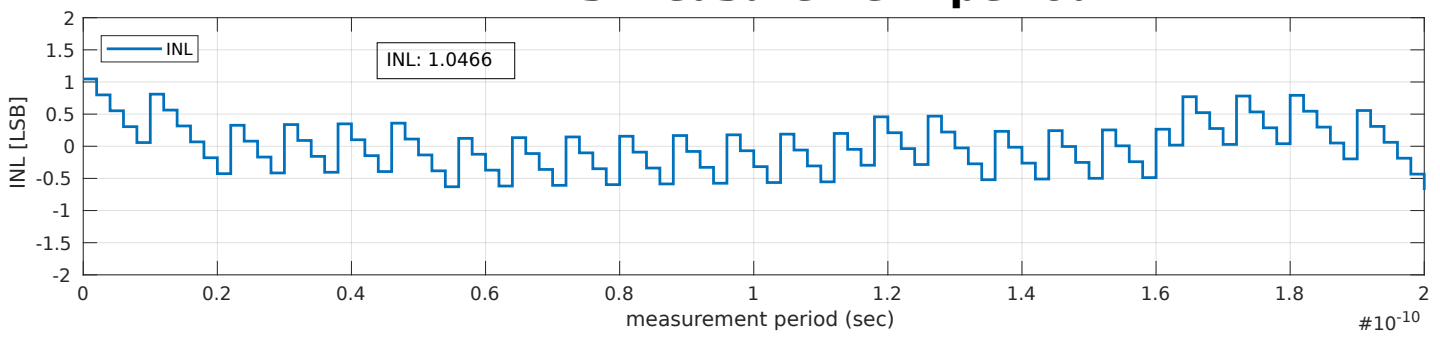

DNL, Hits vs code bin (code density)

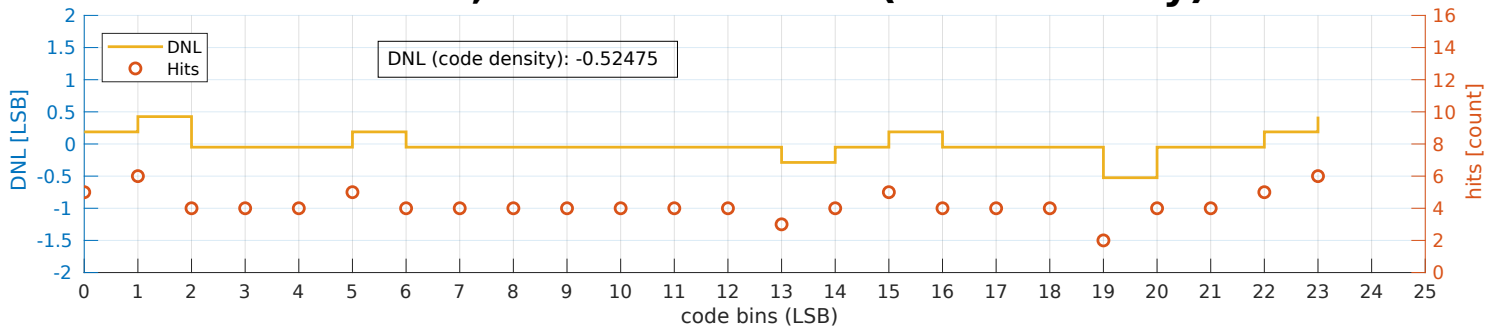

Figure 6.12: Measured LPI-TDC non-linearity at VDD $=1 \mathrm{p} 4 \mathrm{~V}, \mathrm{f}_{\text {start }}=1.6 \mathrm{GHz}$, $\mathrm{f}_{\text {stop }}=100 \mathrm{MHz}$

\subsection{Power Consumption}

The power measurements were performed on both VddBuf and VddInt domains using the Agilent 3648 dual-output supply. A total of 10 consecutive readings are made per each phase step during the experiment and sent to the MATLAB for post-processing. A plot of the averaged power consumption as a function of the test period is presented in Figure 6.13

A correlation between the SSP fluctuations and power measurements was found 
TDC power

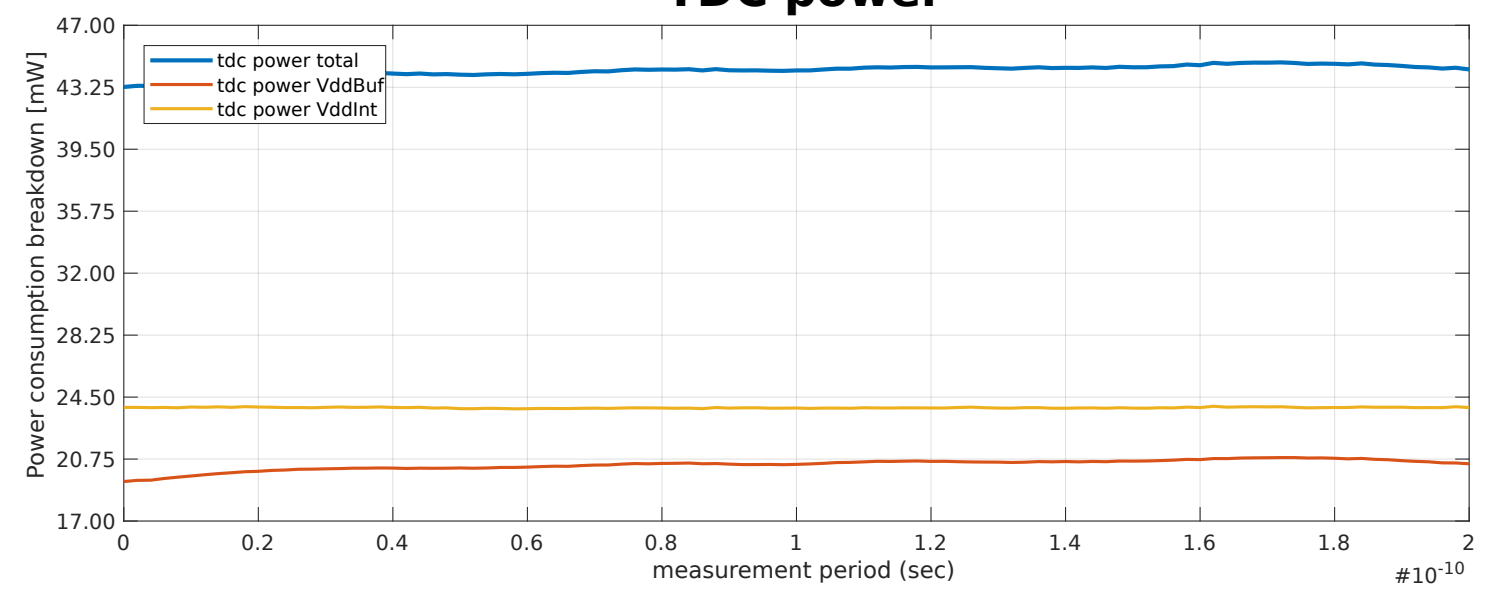

Figure 6.13: Measured power consumption at $\mathrm{VDD}=1.4 \mathrm{~V}, \mathrm{f}_{\text {start }}=1.6 \mathrm{GHz}$, $\mathrm{f}_{\text {stop }}=100 \mathrm{MHz}$

in the measured data Figure 6.14 The on-board power supply was monitored using the oscilloscope and did not reveal any significant PCB board noise. Therefore, the correlation strongly suggests that the on-chip switching noise (SAFF, interpolator, buffers) couple into the supply and contaminate the timing margins of the LPI-TDC during the SAFF latching phase. Further investigation would be required to determine whether the clock feed-through or the kick-back dominate the noise. However, a more pragmatic solution would be to improve the on-chip supply decoupling by adding more local capacitance, reducing the internal metal trace impedances, and using more bond-wires to supply the power.

\subsection{Summary of Measurement Results}

To conclude the chapter, a summary of the results is presented in Table 6.9

Despite the limitation of measured maximum frequency of operation, the proposed LPI-TDC was able to directly quantize the fastest start frequency from what has been reported in the recent literature, exceeding a $1.7 \mathrm{GHz}$. It also does so while achieving 


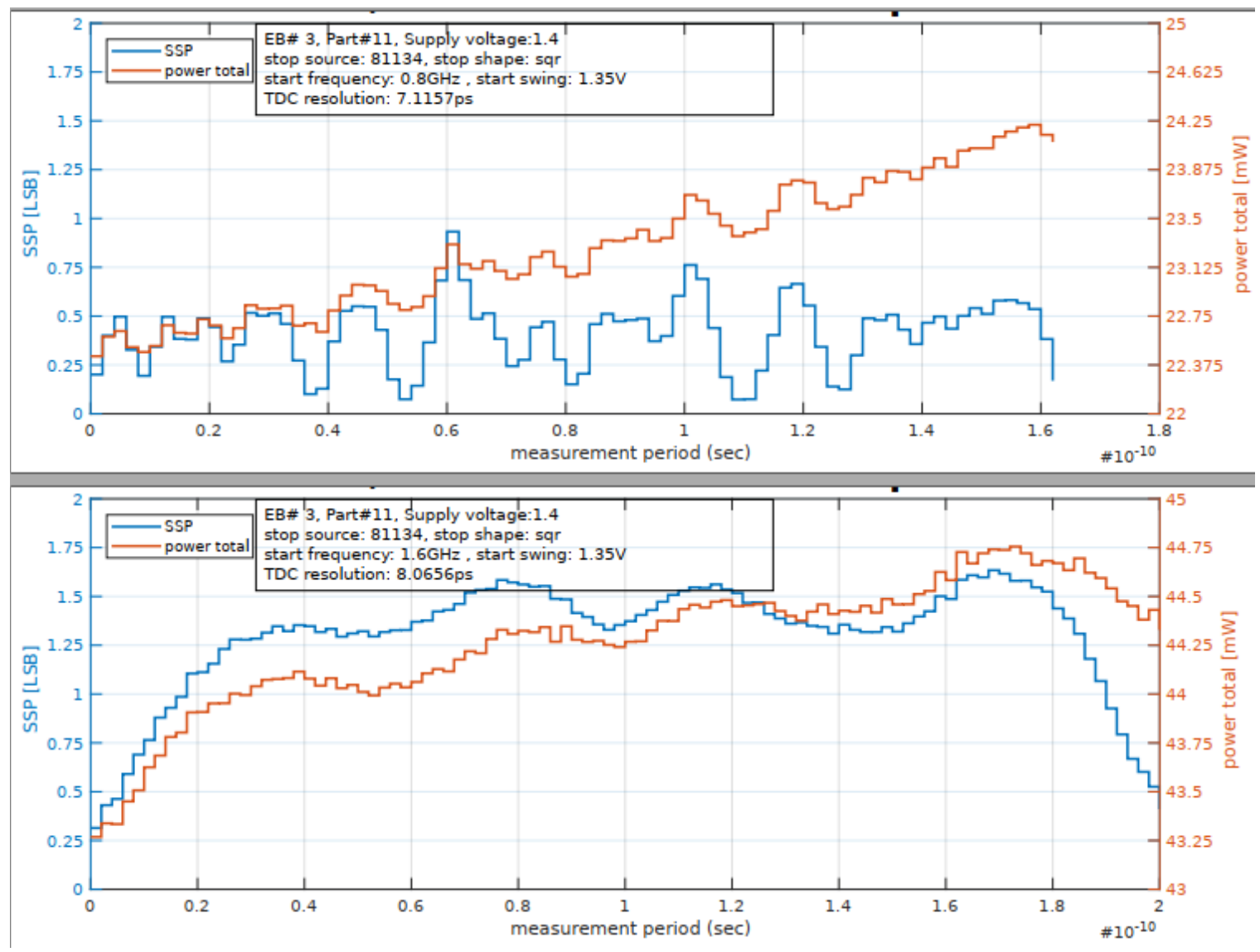

Figure 6.14: Measured power and SSP of Core Card number 3 under various conditions: $\mathrm{VDD}=(1.4 \mathrm{~V}, 1.2 \mathrm{~V}), \mathrm{f}_{\text {start }}=(1.6 \mathrm{GHz}, 0.8 \mathrm{GHz}), \mathrm{f}_{\text {stop }}=100 \mathrm{MHz}$

the sub-gate delay resolution of 8.1 ps with a conversion latency of $<1$ ns (mainly due to the output buffer delays) at a stop rate of $100 \mathrm{MHz}$. The measured SSP of a 1.6 LSB at $1.6 \mathrm{GHz}$ severely reduces the ENOB, but from the ADPLL system perspective it is sufficient for some GSM applications (based on the calculated BFM figure of merit in Table 4.2. Therefore, the proposed design could allow for a direct phase detection in an ADPLL, eliminating the need for a divider.

The architecture is compatible with a looped (Nutt method) dynamic range extension, which would allow the demonstrator chip to achieve a respectable $295 \mathrm{fJ} / \mathrm{bit}$ at $1.6 \mathrm{GHz}$ when such technique is used $[9,17]$. With better attention to the signal and supply integrity, the energy FOM has the potential to be reduced down to 120 
$\mathrm{fJ} /$ bit at $5 \mathrm{GHz}$, which compares well to the other works in this field [27].

The measured INL and DNL figures are acceptable and show that despite the poor noise performance, the architecture remains monotonic.

As demonstrated in Section 6.6, the main limitation of the measured results has to do with the signal integrity of the input signal and the on-chip supply. With the extra care taken during the design stage these limitations can be easily circumvented and the design would be able to directly quantize the claimed $5 \mathrm{GHz}$ start signal. 
Table 6.9: Measured LPI-TDC performance summary.

\begin{tabular}{|c|c|c|c|c|c|c|}
\hline Parameter & units & Specification & $\begin{array}{l}\text { Measured } \\
\text { at } 1.6 \mathrm{GHz}\end{array}$ & $\begin{array}{l}\text { Re-simulated } \\
\text { at } 1.6 \mathrm{GHz}\end{array}$ & $\begin{array}{l}\text { Measured } \\
\text { at } 0.8 \mathrm{GHz}\end{array}$ & $\begin{array}{c}\text { Henlzer } \\
\text { 29 }\end{array}$ \\
\hline Technology & $\mathrm{nm}$ & 130 & 130 & 130 & 130 & 90 \\
\hline $\mathrm{BFM}_{\mathrm{dB}}$ & $\mathrm{dB}$ & -215 & -202 & -206 & -202 & -210 \\
\hline ENOB & LSB & 4.7 & 2.53 & 4.2 & 3.33 & 5.72 \\
\hline $\mathrm{T}_{\mathrm{LSB}, \mathrm{eff}}$ & ps & 9.80 & 44.9 & 14.3 & 22.7 & 11.4 \\
\hline$f_{\text {start }}^{\max }$ & $\mathrm{GHz}$ & 5 & 1.7 & 3 & 1.7 & 0.18 \\
\hline$f_{\text {start }}^{\text {measurement }}$ & $\mathrm{GHz}$ & 5 & 1.6 & 1.6 & 0.8 & 0.18 \\
\hline $\mathrm{f}_{\text {stop }}$ & $\mathrm{MHz}$ & 100 & 100 & 100 & 100 & 180 \\
\hline $\begin{array}{l}\text { Dynamic Range } \\
\text { (fine TDC core) }\end{array}$ & ps & 255 & 259 & 264 & 228 & 600 \\
\hline $\mathrm{T}_{\mathrm{LSB}, \text { design }}$ & ps & 8 & 8.1 & 8.25 & 7.12 & 4.7 \\
\hline $\mathrm{SSP}_{\mathrm{LSB}}$ & LSB & $<1$ & 1.6 & $<0.5$ & 0.9 & 0.7 \\
\hline INL & LSB & $<1$ & 1.02 & 0.86 & 1.06 & 1.2 \\
\hline DNL & LSB & $<0.5$ & 0.52 & 0.43 & 0.76 & 0.6 \\
\hline VDD & $\mathrm{V}$ & 1.40 & 1.40 & 1.40 & 1.40 & 1.2 \\
\hline Temperature & ${ }^{\circ} \mathrm{C}$ & 65 & 65 & 65 & 65 & $\mathrm{n} / \mathrm{a}$ \\
\hline $\begin{array}{l}\text { Power }\left(\propto \mathrm{f}_{\text {start }}\right) \\
\text { (core interpolator\&buffers) }\end{array}$ & $\mathrm{mW}$ & 94.5 & 40.0 & 33.4 & 20.5 & 14 \\
\hline $\begin{array}{l}\text { Power }\left(\propto \mathrm{f}_{\text {stop }}\right) \\
\text { (SAFF\&output buffers) }\end{array}$ & $\mathrm{mW}$ & 10.5 & 4.0 & 6.0 & 3.5 & $\mathrm{n} / \mathrm{a}$ \\
\hline Power total (LPI-TDC ) & $\mathrm{mW}$ & 105.00 & 44.0 & 39.4 & 24.0 & 14.0 \\
\hline $\begin{array}{l}\text { Area } \\
\text { (core,buffer,SAFF) }\end{array}$ & $\mathrm{mm}^{2}$ & $<0.2$ & 0.09 & 0.09 & 0.09 & 0.02 \\
\hline $\begin{array}{l}\mathrm{FOM}_{\mathrm{P}} \\
\text { (fine TDC core) }\end{array}$ & Joules/bit & $4.77 \mathrm{p}$ & $11.3 \mathrm{p}$ & $4.38 \mathrm{p}$ & $6.04 \mathrm{p}$ & $2.65 \mathrm{p}$ \\
\hline Counter $(\mathrm{N})$ & count & 40 & 40 & 40 & 40 & 40 \\
\hline $\begin{array}{l}\text { Power } \\
(\text { counter } \sqrt{17}])\end{array}$ & $\mathrm{mW}$ & $\approx 5$ & $\approx 5$ & $\approx 5$ & $\approx 5$ & $\approx 2$ \\
\hline Dynamic Range with counter & $\mathrm{ns}$ & 10.2 & 10.4 & 10.6 & 9.1 & 10.2 \\
\hline $\begin{array}{l}\mathrm{FOM}_{\mathrm{P}} \\
\text { (coarse counter \&fine TDC core) }\end{array}$ & Joules/bit & $120.1 \mathrm{f}$ & $295 \mathrm{f}$ & $113.7 \mathrm{f}$ & $166.5 \mathrm{f}$ & $179.7 f$ \\
\hline $\begin{array}{l}\text { FOM }_{\text {PAC }} \\
\text { (coarse counter \&fine TDC core) }\end{array}$ & $\$ \cdot$ Joules / bit & $48.1 \mathrm{p}$ & $52.5 \mathrm{p}$ & $20.2 \mathrm{p}$ & $29.6 \mathrm{p}$ & $10.1 \mathrm{p}$ \\
\hline
\end{tabular}




\section{Chapter 7}

\section{Conclusion}

\subsection{Contributions to Research}

In this work, the design of a 5-bit TDC based on the Linear Passive Interpolation technique was presented including system level specifications, circuit schematic and layout design, and measured performance of the manufactured device. A custom library of mixed signal and digital circuits was created in IBM $0.13 \mu \mathrm{m}$ process which included inverter-based interpolation delay cells, sense amplifier (comparators), setreset latches, and buffers. The performance was simulated using the built-in Cadence tools using models provided by the foundry. A challenge of signal integrity was mitigated in measurements through a careful design of the test setup and the custom printed circuit board. The measured results closely match the predicted behaviour, showing an $8.1 \mathrm{ps}$ resolution at a $1.4 \mathrm{~V}$ supply with a $1.6 \mathrm{GHz}$ input start frequency. The achieved rate is the highest measured, and with some adjustments can exceed the fastest simulated in the recently reported literature 30,31 . Given an inherently digital nature of the circuit, the trade-off between power supply, resolution, and the maximum achievable interpolation frequency was examined. Several contributions have been made in the process. 
A fine resolution time-to-digital converter was designed to directly convert a multiGHz signal at a $100 \mathrm{MHz}$ sampling rate in a mature $0.13 \mu \mathrm{m}$ process. The passive interpolation technique combines the low conversion latency (dead time), sub-gatedelay resolution sufficient for GSM applications, and an inherently robust linearity. The fundamental limits of resolution and linearity are set mainly by the on-chip circuit parasitics, power requirements, as well as signal and supply integrity of the design.

The demonstrator chip has successfully converted a $1.7 \mathrm{GHz}$ input start signal at a rate of $100 \mathrm{MHz}$. The upper frequency limit is capped mainly by the input signal and power supply integrity. With a better input matching and supply impedance control, the LPI-TDC can quantize the start signal of up to $5 \mathrm{GHz}$, evident from a strong correlation between measured and simulated results.

The measured INL is $1.02 \mathrm{LSB}$ and DNL is $0.52 \mathrm{LSB}$ observed at the $1.6 \mathrm{GHz}$ input frequency. A series of experiments have shown that measured linearity can be further improved by applying a narrower phase difference between the start and the stop signals. However, given the available equipment and methodology, it would take weeks to complete a single sweep, as opposed to just several hours for coarser step size presented in this work. A full bench automation is required in such case, however, a different set of equipment would have to be used to enable such method. With a fine input step size, it should be feasible to achieve the INL and DNL $<0.6$ LSB. An even further improvement is expected from addressing the input matching and the quality of the internal supply.

The single-shot-precision (SSP) of 1.6 LSB is measured at $1.6 \mathrm{GHz}$ and drops to $0.9 \mathrm{LSB}$ at lower frequencies, indicating that this parameter can also be reduced further with an improved signal conditioning.

Configured in a looped architecture (Nutt method), the energy efficiency of about $295 \mathrm{fJ} /$ bit is predicted and it has a potential to be reduced to $120 \mathrm{fJ} /$ bit. Therefore, 
this work shows that it is feasible to have an energy efficiency FOM comparable to the state of the art designs, while achieving a low conversion time and a sufficient dynamic range (DR) for a broad range of fast ADPLL applications.

Given the ability to process the input frequencies in a multi-GHz range, the proposed LPI-TDC design can operate without the need for a feedback divider. The fast TDC also enables the use of very large ADPLL loop bandwidths for fast locking and can also be exploited in a locked state (tracking mode) to reduce the jitter peaking, since a high effective loop BW (error update rate) gives more room for the integral and proportional path optimization.

Measurement techniques such as swept phase and ways to reduce the effect of instrumentation jitter are also provided in this work.

\subsection{Future Work}

Based on the measured and re-simulated results, it became apparent that the design can benefit from an improved signal integrity for a stand-alone characterization to measure frequencies in the desired range of $5 \mathrm{GHz}$ and have a low system noise (SSP).

The demonstrator chip used two separate power domains (VddBuf and VddInt). However, both supplies are at the same potential, and with a good off-chip supply decoupling network, similar to what has been implemented on the evaluation board (Section A.1.4, the DUT can operate from a single power source. Moreover, to make the design more practical, with an improved on-chip supply decoupling strategy, only a single domain can be sufficient.

A better supply integrity would also reduce the bubble errors and improve the SSP metric (that would also improve the ENOB and FOM). For additional improvements to the SSP, the noise coupling between the interpolation core and the SAFF should be 
further reduced with a better buffer design that provides superior kick-back isolation (i.e. graceful domain crossing). To reduce the amount of instantaneous SAFF switching noise coupling into the supply, the dummy SAFF loads with opposite polarities can be placed at the expense of area and some average power consumption.

To extend the LPI-TDC dynamic range and improve the energy efficiency, this design can be slightly modified to work in a loop configuration (the Nutt method) or paired with a fast coarse counter.

The LPI-TDC on-chip footprint can be further reduced by optimizating the delay stage layout and the SAFF. The floorplan proposed in this work was very conservative and can be modified to use less than half of the area shown in the demonstrator chip. To enable an even deeper design scaling, interpolation resistors with more optimal sheet resistivity (e.g. tantalum nitride) could also be considered. Such optimization would also significantly improve the $\mathrm{FOM}_{\mathrm{PAC}}$.

An alternative investigation has started by looking at the delay cell that is based on a fully-differential current-mode logic (CML) amplifier. The preliminary design simulations have shown good feasibility to achieve power reduction by an order of magnitude $(10 \mathrm{x})$ lower compared to the proposed implementation, while operating at $5 \mathrm{GHz}$. Although such design choice diverges from an all-digital implementation, some applications may find this method useful, given the inherent simplicity, robustness, and the good linearity of the passive interpolation principle. 


\section{List of References}

[1] CMCmicrosystems, "CMC fabrication and pricing quotes." https://www.cmc. ca/en/WhatWeOffer/Make/FabPricing.aspx, accessed March 10, 2019.

[2] R. J. Baker, CMOS: Circuit Design, Layout, and Simulation, Revised, 2nd Edition. John Wiley \& Sons, 2007.

[3] S. Henzler, Time-to-Digital Converters. Springer Netherlands, 2010.

[4] R. B. Staszewski and P. T. Balasara, All-digital frequency synthesizer in deepsubmicron CMOS. Wiley-Interscience, 2006.

[5] J. W. Rogers and C. Plett, Radio frequency integrated circuit design. Artech House Publishers, 2010.

[6] J. Borremans, K. Vengattaramane, V. Giannini, B. Debaillie, W. Van Thillo, and J. Craninckx, "A $86 \mathrm{MHz} 12 \mathrm{GHz}$ digital-intensive PLL for software-defined radios, using a $6 \mathrm{fJ} / \mathrm{step}$ TDC in $40 \mathrm{~nm}$ digital CMOS," IEEE Journal of SolidState Circuits, vol. 45, pp. 2116-2129, Oct 2010.

[7] M. Rashdan, "Multi-step and high-resolution vernier-based TDC architecture," in 2017 29th International Conference on Microelectronics (ICM), pp. 1-4, Dec 2017.

[8] J. Yu, F. F. Dai, and R. C. Jaeger, "A 12-bit vernier ring time-to-digital converter in $0.13 \mu \mathrm{m}$ CMOS technology," IEEE Journal of Solid-State Circuits, vol. 45, pp. 830-842, April 2010.

[9] P. Keranen and J. Kostamovaara, "Noise and nonlinearity limitations of time-tovoltage based time-to-digital converters," in 2013 IEEE Nordic-Mediterranean Workshop on Time-to-Digital Converters (NoMe TDC), pp. 1-6, Oct 2013. 
[10] G. Manganaro and A. Pulincherry, "A behavioral modeling approach to the design of a low jitter clock source," IEEE Transactions on Circuits and Systems II: Analog and Digital Signal Processing, vol. 50, pp. 804-814, Nov 2003.

[11] P. Gjurovski, M. Wei, and R. Negra, "Subsampling phase-locked loop behavioural modelling approach for phase noise evaluation," in 2017 IEEE Nordic Circuits and Systems Conference (NORCAS): NORCHIP and International Symposium of System-on-Chip (SoC), pp. 1-4, Oct 2017.

[12] W. F. Egan, Phase-Lock Basics, 2nd Edition. John Wiley \& Sons, 2007.

[13] P. Napolitano, A. Moschitta, and P. Carbone, "A survey on time interval measurement techniques and testing methods," in 2010 IEEE Instrumentation Measurement Technology Conference Proceedings, pp. 181-186, May 2010.

[14] V. N. Nguyen and J. Lee, "A low power two-step cyclic time-to-digital converter without startup time error in $180 \mathrm{~nm}$ CMOS," in 2018 2nd International Conference on Recent Advances in Signal Processing, Telecommunications Computing (SigTelCom), pp. 116-120, Jan 2018.

[15] Y. Wu, P. Lu, and R. B. Staszewski, "A 103fs rms 1.32mw 50MS/s 1.25MHz bandwidth two-step flash- $\Delta \Sigma$ time-to-digital converter for ADPLL," in 2015 IEEE Radio Frequency Integrated Circuits Symposium (RFIC), pp. 95-98, May 2015.

[16] Q. Gu, RF system design of transceivers for wireless communications. Springer, 2005.

[17] K. Kuo and F. Wu, "A 2.4-GHz/5-GHz low power pulse swallow counter in 0.18um CMOS technology," in APCCAS 2006 - 2006 IEEE Asia Pacific Conference on Circuits and Systems, pp. 214-217, Dec 2006.

[18] T. Zhang and Q. Hu, "A high-speed and low-power up/down counter in 0.18-um cmos technology," in 2012 International Conference on Wireless Communications and Signal Processing (WCSP), pp. 1-3, Oct 2012.

[19] D. Morrison, D. Delic, M. R. Yuce, and J. Redout, "Multistage linear feedback shift register counters with reduced decoding logic in 130-nm CMOS for largescale array applications," IEEE Transactions on Very Large Scale Integration (VLSI) Systems, vol. 27, pp. 103-115, Jan 2019. 
[20] U. Vollenbruch, Y. Liu, T. Bauernfeind, T. Pittorino, V. Neubauer, T. Mayer, and L. Maurer, "Requirements for time-to-digital converters in the context of digital-PLL based frequency synthesis and GSM modulation," in 2006 IEEE MTT-S International Microwave Symposium Digest, pp. 1817-1820, June 2006.

[21] R. B. Staszewski, S. Vemulapalli, and K. Waheed, "An all-digital offset PLL architecture," in 2010 IEEE Radio Frequency Integrated Circuits Symposium, pp. 17-20, May 2010.

[22] N. H. E. Weste and D. Harris, CMOS VLSI design: a circuits and systems perspective. Pearson/Addison-Wesley, 2005.

[23] B. Nikolic, V. G. Oklobdzija, V. Stojanovic, , , and M. Ming-Tak Leung, "Improved sense-amplifier-based flip-flop: design and measurements," IEEE Journal of Solid-State Circuits, vol. 35, pp. 876-884, June 2000.

[24] Z. Jaworski, "Verilog HDL model based thermometer-to-binary encoder with bubble error correction," in 2016 MIXDES - 23rd International Conference Mixed Design of Integrated Circuits and Systems, pp. 249-254, June 2016.

[25] V. H. Bui and T. T. Jeong, "Thermometer-to-binary encoder with bubble error correction (BEC) circuit for flash analog-to-digital converter (FADC)," in International Conference on Communications and Electronics 2010, pp. 102-106, Aug 2010.

[26] B. Wicht, T. Nirschl, and D. Schmitt-Landsiedel, "Yield and speed optimization of a latch-type voltage sense amplifier," IEEE Journal of Solid-State Circuits, vol. 39, pp. 1148-1158, July 2004.

[27] S. Henzler, S. Koeppe, W. Kamp, H. Mulatz, and D. Schmitt-Landsiedel, "90nm 4.7ps-resolution 0.7-LSB single-shot precision and 19pJ-per-shot local passive interpolation time-to-digital converter with on-chip characterization," in 2008 IEEE International Solid-State Circuits Conference - Digest of Technical Papers, pp. 548-635, Feb 2008.

[28] PCBway, "HK weiku information \& technology co., ltd," 2017. https://www. pcbway.com/; accessed March 20, 2019.

[29] S. Henzler, S. Koeppe, D. Lorenz, W. Kamp, R. Kuenemund, and D. SchmittLandsiedel, "A local passive time interpolation concept for variation-tolerant 
high-resolution time-to-digital conversion," IEEE Journal of Solid-State Circuits, vol. 43, pp. 1666-1676, July 2008.

[30] S. Jia, L. Weng, W. Wang, and Y. Wang, "A highly linear 5GS/s Voltage-toTime Converter for time-based Analog-to-Digital Converters," in 2017 IEEE Asia Pacific Microwave Conference (APMC), pp. 714-717, Nov 2017.

[31] L. Perktold and J. Christiansen, "A flexible 5 ps bin-width timing core for next generation High-Energy-Physics Time-to-Digital Converter applications," in PRIME 2012; 8th Conference on Ph.D. Research in Microelectronics Electronics, pp. 1-4, June 2012.

[32] P. Instruments, "Si9000e PCB field solver software tool provider," 2019. https: //www.polarinstruments.com/; accessed March 20, 2019.

[33] K. Kundert, "Power supply noise reduction," tech. rep., Jan 2004. 


\section{Appendix A}

\section{PCB Design Details}

\section{A.1 Core Card Design}

The purpose of a core card is to provide an assembly footprint for the DUT, route highspeed signals, deliver power, and route the TDC output code for further processing by a logic analyzer. The core card requires good signal integrity in order to propagate signals in multi-Gigahertz frequency range. For this reason, the 4-layer process with ENIG finish was favoured over a cheaper 2-layer stack-up. The overall core size was kept under $5 \times 10 \mathrm{~cm}$ to keep the low cost of manufacturing and avoid board warping in the re-flow oven.

The design of critical board interfaces such as the high-speed input, clock management, 24-bit wide output bus, and power delivery are discussed in Section A.1.1 Section A.1.2, Section A.1.3, and Section A.1.4 respectively. 


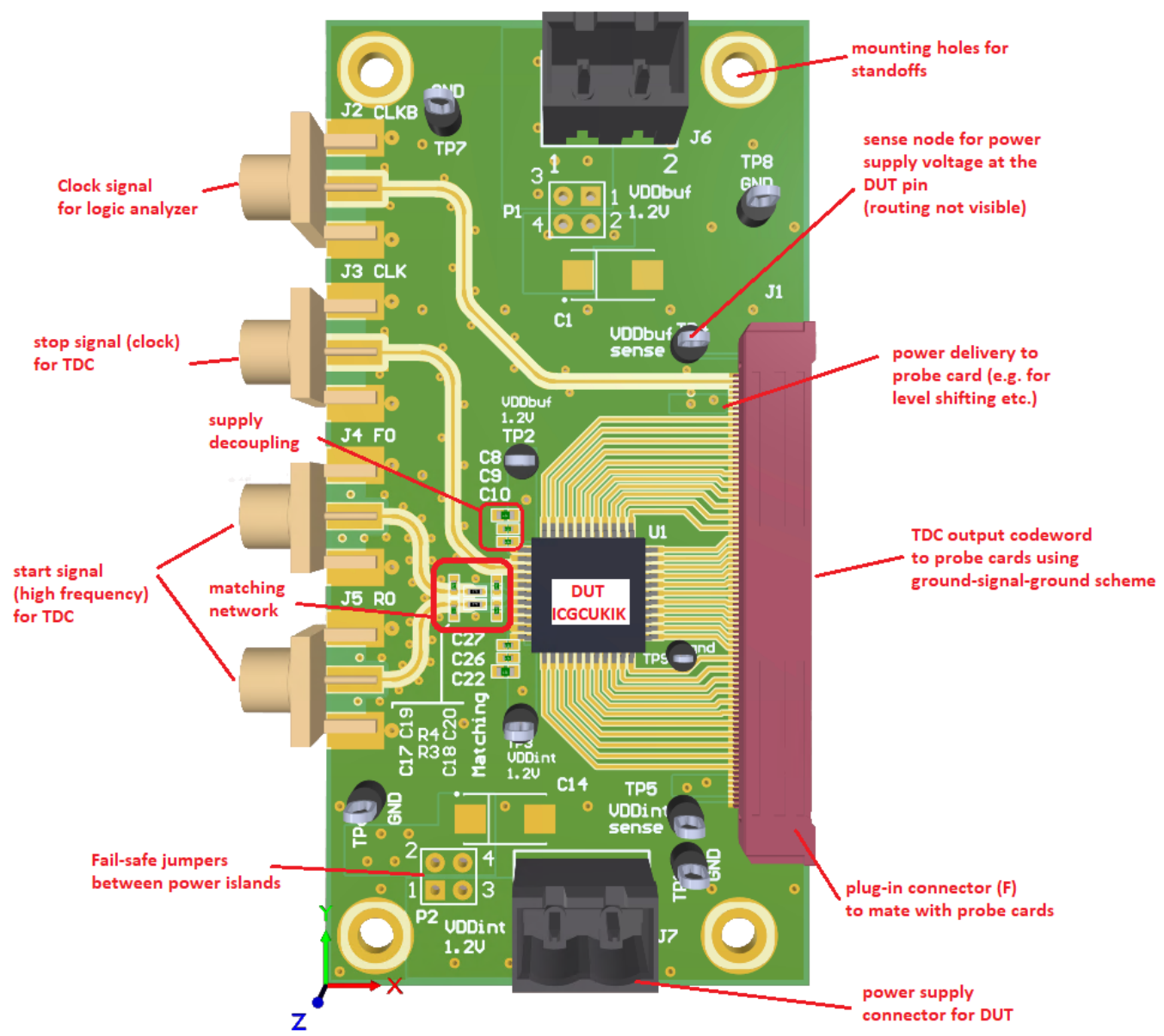

Figure A.1: Annotated core card top side view (image from Altium Designer v17)

\section{A.1.1 High Speed Input (Start Signal)}

The start inputs of TDC are driven by a 50-Ohm source using cables with 50-Ohm characteristic impedance. The upper frequency of operation is expected to be approximately $5 \mathrm{GHz}$. The impedance looking into the chip should also be close to $50 \mathrm{Ohms}$ for optimal power delivery and reduce reflections. However, there is no on-chip termination to guarantee the optimal power transfer. Therefore, an off-chip input impedance matching network was designed to transform chip input impedance into $50 \mathrm{Ohm}$ across a range of frequencies (mainly in the vicinity of $1-5 \mathrm{GHz}$ where fundamental is expected to be). 


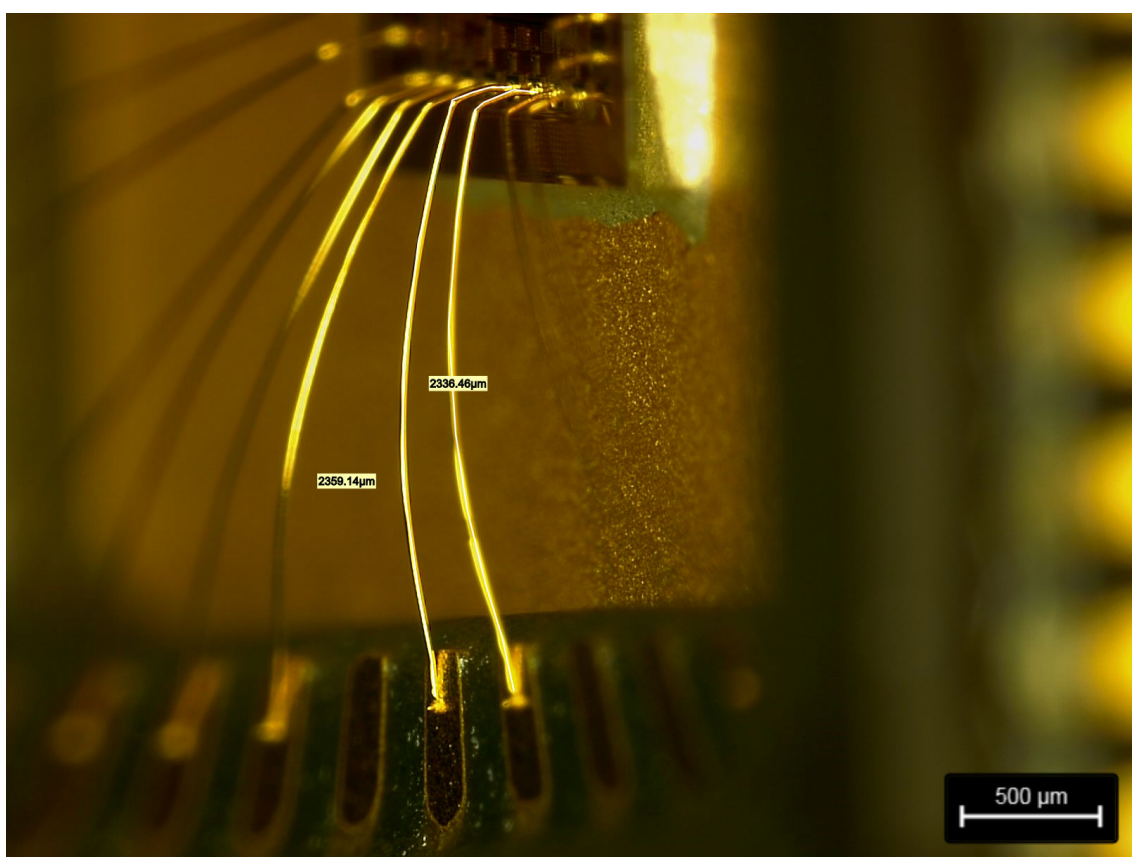

Figure A.2: Measuring bond-wire length under the microscope (Leica DVM6A).

Placement of such network has to be as close to the package input as possible. Therefore, some length of input traces will exist between the input matching network and PCB input port connector. The relative permittivity of FR4 dielectric is $\approx 4$, taken from the manufacturer's process stack-up specification [28. Therefore, the wavelength is $\lambda=\frac{c}{f \sqrt{\epsilon_{r}}}=\frac{3 e 8}{5 e 9 \sqrt{4}}=0.03 \mathrm{~m}$. To avoid signal degradation due to reflections, the maximum on-board trace lengths should be less than $\frac{1}{10}$ of the wavelength, i.e. $3 \mathrm{~mm}$. Designing a board with trace lengths $<3 \mathrm{~mm}$ is not possible for this PCB. Therefore, input traces are treated as controlled-impedance lines and are designed to be $\mathrm{Z}_{0}=50 \Omega$ characteristic impedance.

The input impedance of chip including package and bond-wire was re-simulated with improved interconnect models and parasitics, based on actual measurements done under the microscope (Figure A.2).

The simulation results in Figure A.3 indicate possible self-resonance at frequencies 


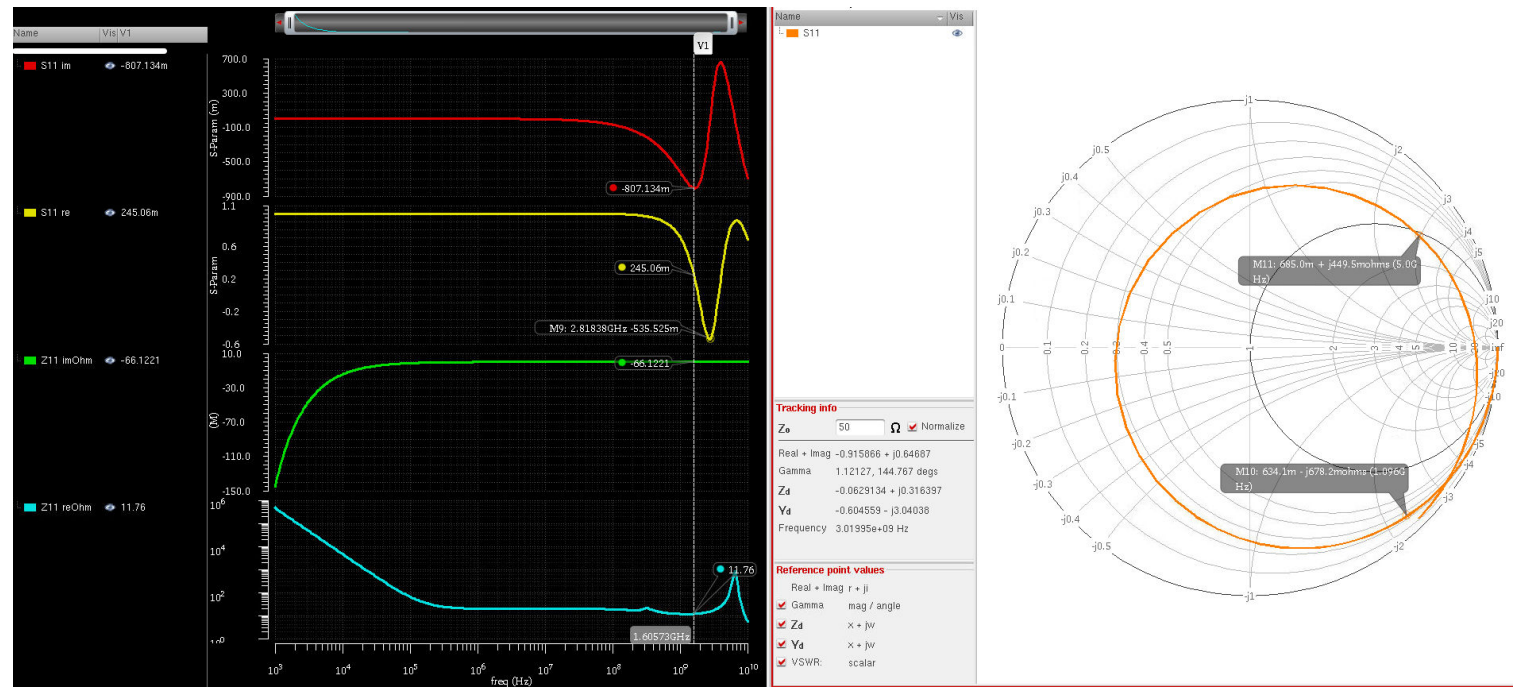

Figure A.3: ICGCUKIK input impedance simulation with updated models based on measured interconnect dimensions.

$>1.6 \mathrm{GHz}$. The exact frequency may vary as it is limited by the model accuracy. Ideally a separate dedicated PCB is needed to de-embed the input impedance through measurement. However due to cost and schedule impact this approach was replaced with a more experimental method.

Using a software tool called Smith v4.0, the simulated S-parameter data was used to generate matching network structures. The design was intentionally limited to 3element narrow-band structure to minimize the complexity and assembly. Based on calculation from Smith v4.0, the optimal matching network that minimizes reflections between $1 \mathrm{GHz}$ and $5 \mathrm{GHz}$ is a series $\mathrm{R}=50 \Omega$ followed by a shunt parallel capacitor $\mathrm{C}=2.7 \mathrm{pF}$ to ground (Figure A.4). This topology was favoured over other options to allow accurate control of DC bias (common mode) for start signal. The series resistance also helps dissipate some energy of the possible reflections. The RF grade passive components were ordered to make sure the matching network self-resonance is higher than start frequency of operation. 


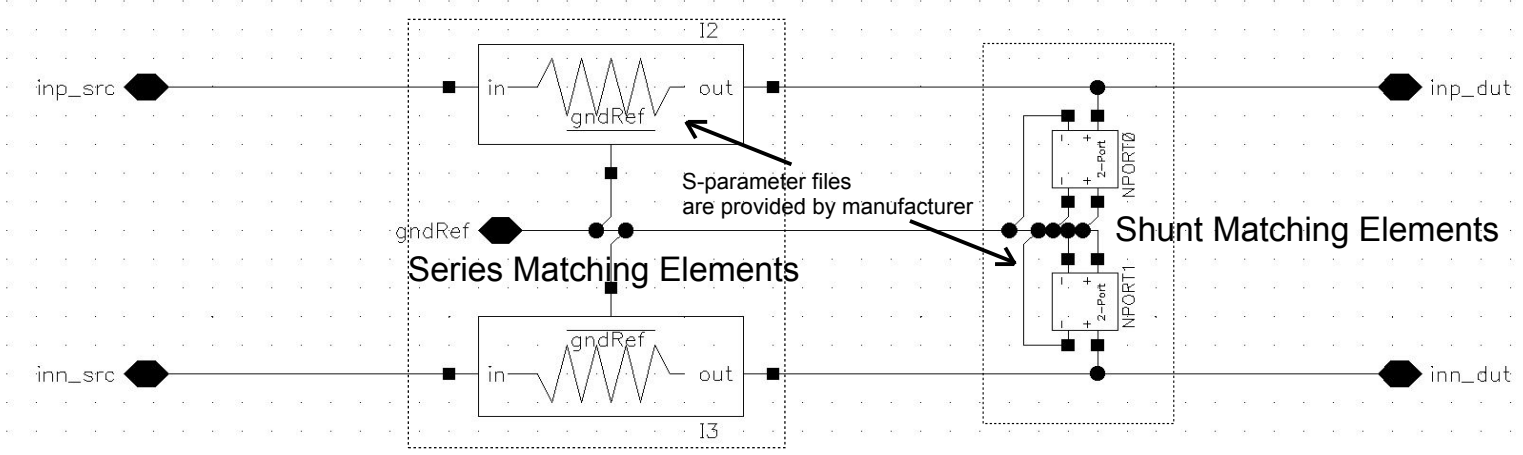

Figure A.4: PCB input matching network with accurate S-parameter models is included in top-level simulation to assess its effectiveness.

The component datasheets were carefully studied to ensure self-resonant frequencies are maximized. Accurate s-parameter models for discrete passive components were obtained from manufacturer and included in test bench to verify expected signal integrity improvements in simulations. The simulation results in Figure A.5 show improvement using the proposed matching network as impedance is much closer to desired 50Ohms across a wide range of frequencies, achieving input reflection $\mathrm{S} 11<-$ $10 \mathrm{~dB}$ at up to $7.5 \mathrm{GHz}$. The input impedance simulations assume $50 \Omega$ characteristic
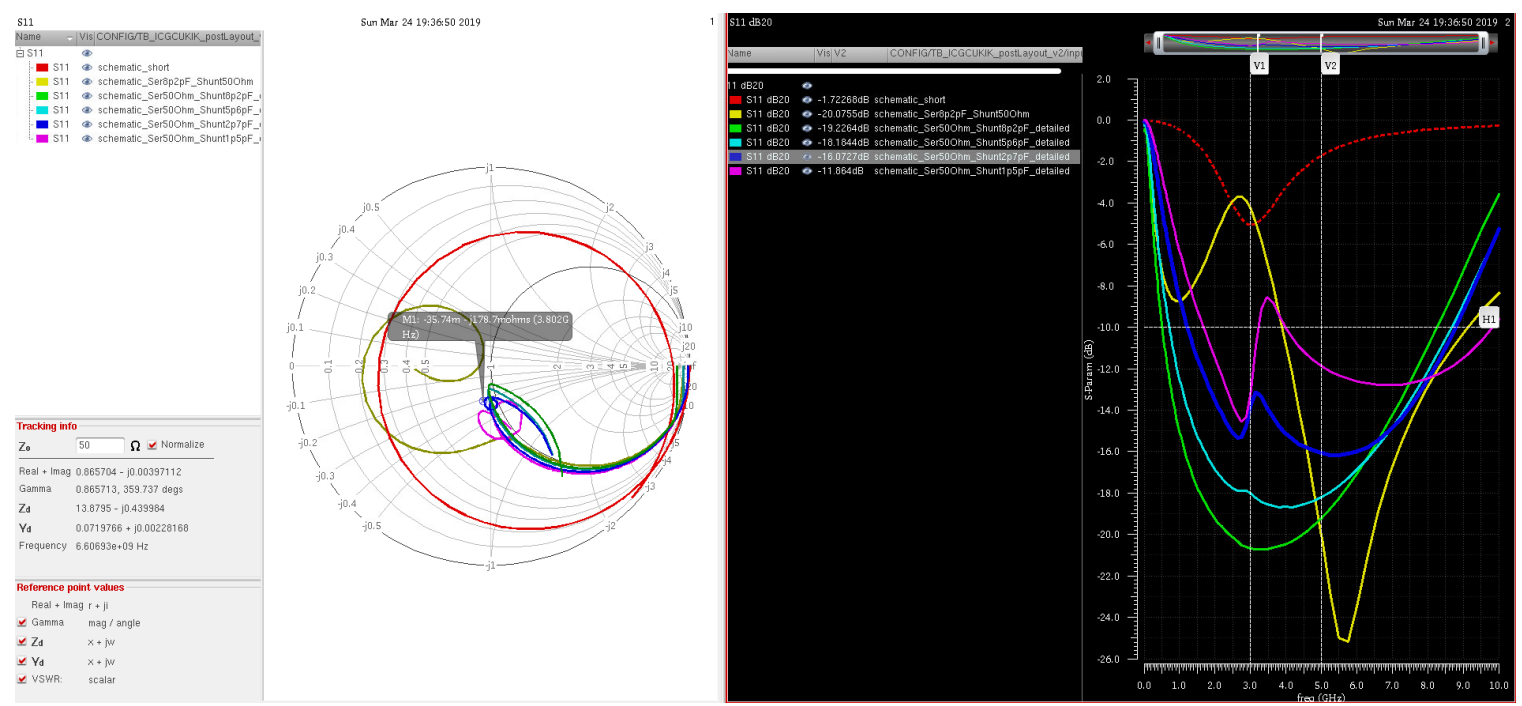

Figure A.5: ICGCUKIK input impedance simulation with updated models based on measured interconnect dimensions 


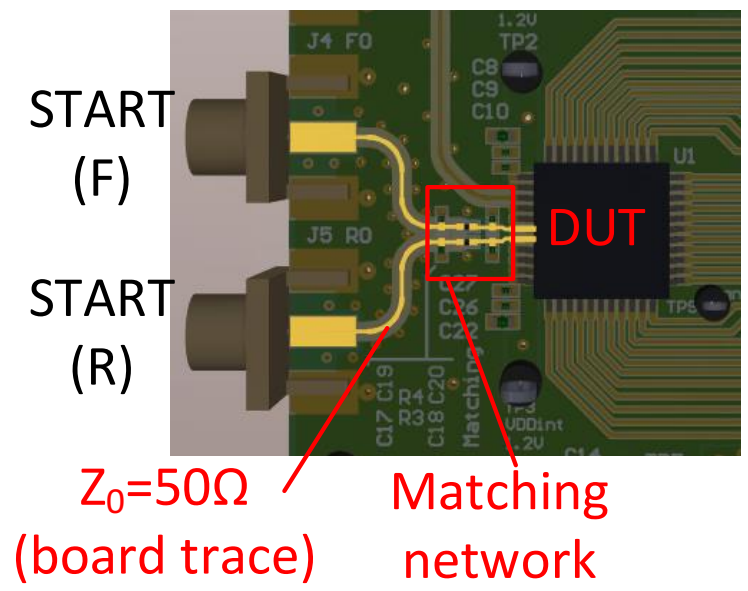

Figure A.6: Input matching network and high speed start signal path layout

impedance up to the matching network input. Therefore, all traces board traces must guarantee it for matching network to be effecive.

The input PCB traces were designed using Polar software tools [32]. To allow for easier debug, it was decided to keep the input traces not covered by solder mask for probe access as well as simplified re-work. The trade-off for not using solder mask is the added complexity during the assembly (i.e. soldering RF components to long exposed metal traces). From calculations using Polar software, it became apparent that a 4-layer board allows usage of narrower traces to achieve the same 50-ohm impedance, compared to a 2-layer board. The narrower traces allow better routing flexibility, board design, and assembly since some RF discrete components have to be soldered. The Electroless Nickel Immersion Gold (ENIG) finish was also favoured over the Hot Air Solder Leveling (HASL) for better high-speed performance due to a lower parasitic lumped resistance of trace lines and less frequency-dependent skin effect.

All high-speed traces are shielded by small-pitch board via stitching to minimize ground return paths as seen in Figure A.6 


\section{A.1.2 Sampling Clock and Stop Signals}

The clock signals are needed in the system for two purposes: provide stop signal for TDC and synchronous sampling clock for logic analyzer. For testing, redundancy, cable management, and debug purposes, it was decided to route both on core card. A delay (phase shift) between the two signals is required to allow TDC output settle prior to capturing its state by logic analyzer. Taking advantage of function generator (Agilent 81150 or 81134 ) complimentary outputs provides inherent $180^{\circ}$ offset. This translates to $\frac{1}{100 \exp 6 \times 2}=5 n s$ requirement for TDC outputs to settle $\left(\frac{1}{2}\right.$ the clock period) and propagate from the chip to logic analyzer. Simulation results estimated the LPI-TDC latency to be $\approx 900 p s$, which leaves $4 \mathrm{~ns}$ margin to cover propagation delay through cables (Figure 5.21). An additional offset can be manually dialed in measurement to provide more timing margin between if needed.

Just like the high speed input path, the stop signal quality was considered. Fortunately, running the clock at $100 \mathrm{MHz}$, relaxes design constraints. The electrical

wavelength is $\approx 1.5 \mathrm{~m}\left(\frac{3 \exp 8}{100 \exp 6 * \sqrt{2}}\right)$, which translates to maximum un-terminated length allowance of $15 \mathrm{~cm}\left(\lambda \frac{1}{10}\right)$.

The cables between clock generator and board are approximately $1 \mathrm{~m}$ in length. Therefore, the far-end termination is desirable and should be placed as close to the device under test as possible, especially given that the device under test does not have an on-chip termination. The solution is to keep the PCB traces less than $15 \mathrm{~cm}$ long and leave enough access to solder a $50 \Omega$ resistor if required. The logic analyzer sampling clock is also kept under $15 \mathrm{~cm}$ in length and was given an termination option through redundant SMA ports using screw-on termination on probe cards discussed in Section A.2. 


\section{A.1.3 TDC Output Word}

The thermometer coded LPI-TDC output is a 24-bit wide signal bus that requires some care to route to be probed. On the one hand, it is desired to keep all traces short and narrow to reduce loading for faster switching speed, smaller PCB footprint (area and cost savings). On the other hand, it is desirable to minimize the crosstalk between the adjacent tracks and reflections to/from the logic analyzer. To limit the cross-talk, the outputs are routed using ground-signal-ground (GSG) as seen in Figure A.7. The signal width and separation between adjacent signals were chosen to match the board-to-board connector pitch.

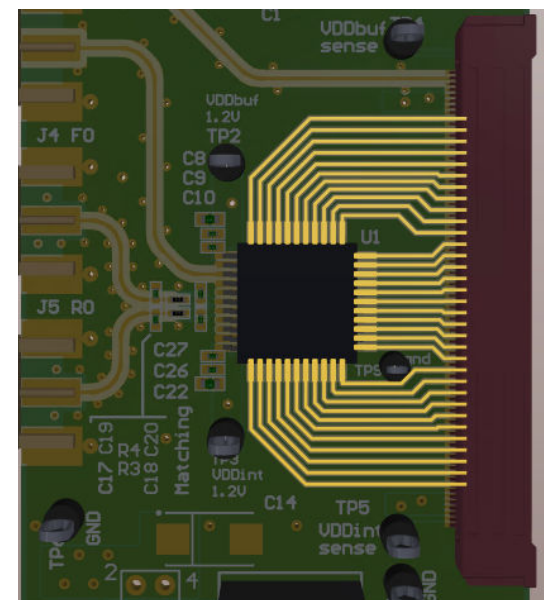

Figure A.7: ICGCUKIK LPI-TDC outputs routed in GSG configuration to be probed by logic analyzer

The following items were considered to select the board-to-board connector:

- suitable for signal integrity (i.e. controlled impedance)

- small footprint, but large enough for manual assembly and probing

- contains >60 pins to handle 24 bit wide LPI-TDC output in GSG configuration and power

- support large number of connect/disconnect cycles 
- has locking mechanism to maintain secure connection during measurement

- surface-mounted solution to avoid complex multi-layered probe card design

- part cost

Based on these requirements, a Hirose 80-pin connector FX-22 was selected.

\section{A.1.4 Power Delivery}

The two power domains VddInt and VddBuf are intentionally kept separated on PCB to provide independent supply options and measurements. To minimize the board impedance, power is routed using wide tracks and dedicated supply planes on inner PCB layers, achieving inductance «1nH (Figure A.8). For debug and safety, the tracks are further divided into islands connected using removable low-impedance jumpers.

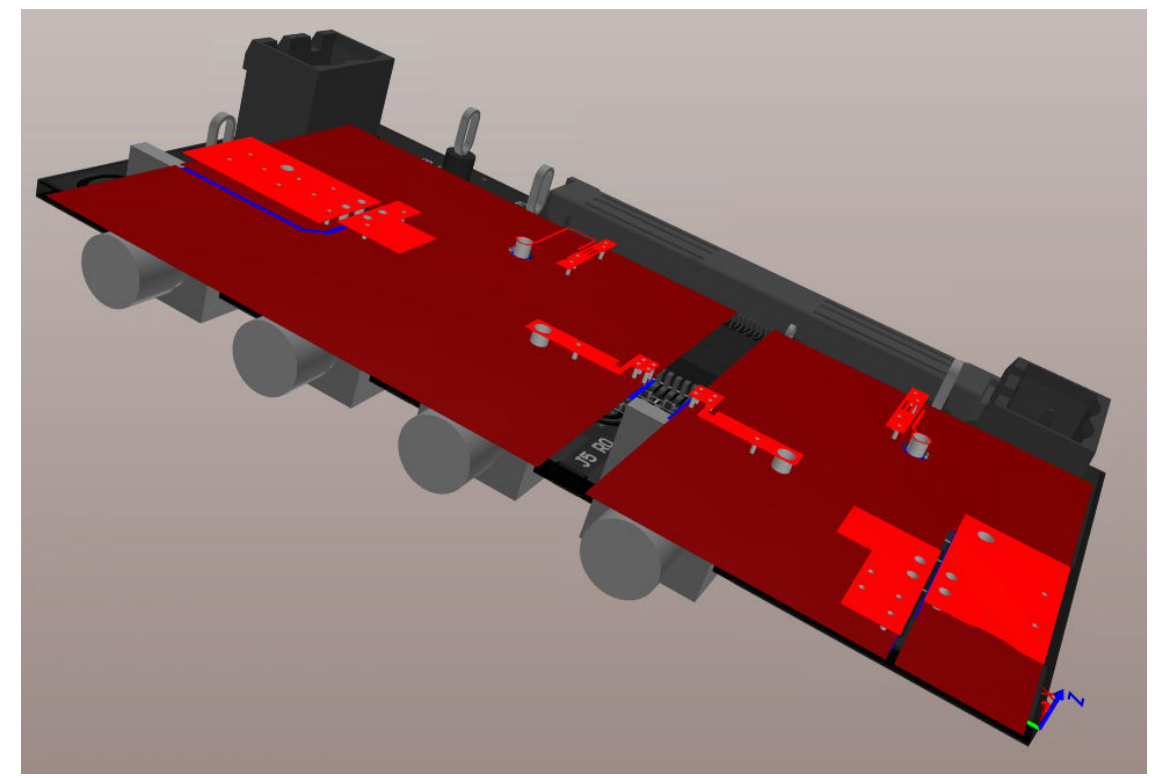

Figure A.8: PCB power planes (darker shade) and top layer tracks (lighter shade) shown with respect to ICGCUKIK and board-to-board connector.

An entire layer was also dedicated to the ground plane which serves the purpose of low-impedance return path for all signals and power. To further minimize the return 
path impedance, all domains are abundantly strapped using vias, uniformly placed across the entire area of the PCB. For redundancy and probing, multiple test points were designed into the PCB. A few access nodes were connected to the low-impedance power plane at inner layers, to serve as alternative method of power delivery to the DUT and attach oscilloscope to monitor the supply noise. A dedicated sense line at the DUT pins was also placed in star configuration. The sense line allows monitoring of supply absolute level at the DUT pins to assess voltage drop due to finite supply resistance. All power, ground, and supply sense domains were routed to the boardto-board connector for possible bi-directional probing and supply options from the probe cards.

The board decoupling capacitors are placed as close to the chip leads as possible on the top and bottom sides of the PCB directly underneath the device. Capacitor placement was distributed along the power plane path. The largest 2917 (330uF) Tantalum capacitors were placed further away from the device due to their size and limited performance, since such capacitors have a lower self-resonant frequency. Their main purpose is to act as charge buffer and filter low-frequency noise originating at either supply or the DUT. They were placed on the DUT side of power plane in case the jumper and wire inductance are higher than expected.

Capacitors with smaller dimensions such as $04020.01 \mathrm{uF}$ were placed close to the DUT pins. These capacitors were selected due to higher quality of dielectric materials and construction, at the expense of smaller absolute capacitance. This helps buffer and filter noise at higher frequencies near the DUT (ICGCUKIK). Such distributed topology and usage of discrete components of various dimensions and dielectric properties allows the capacitive decoupling to remain effective across a broad range of frequencies 33. 


\section{A.2 Probe Card Design}

The probe card is a $\mathrm{PCB}$ which is designed to interface with the core card through a board-to-board connector. The modular architecture enables design of different probe card variants to support a wide selection of test equipment for measurements. The board-to-board connector pin assignment was designed to make sure that all important signals (TDC output, sampling clock), power domains, ground, and sense lines are available for access by the probe card.

\section{A.2.1 Probe Card V1}

The primary purpose of this probe card variant is to enable connectivity with the 16715 logic analyzer. This board was designed to provide a robust fall-back alternative in case probe card v2 doesn't work (Figure A.9). The 20-pin 0.1" connector has been widely-adopted by the industry for many decades. Therefore, this connector also serves a purpose of generic interface for a multitude of equipment options besides the Agilent logic analyzer.

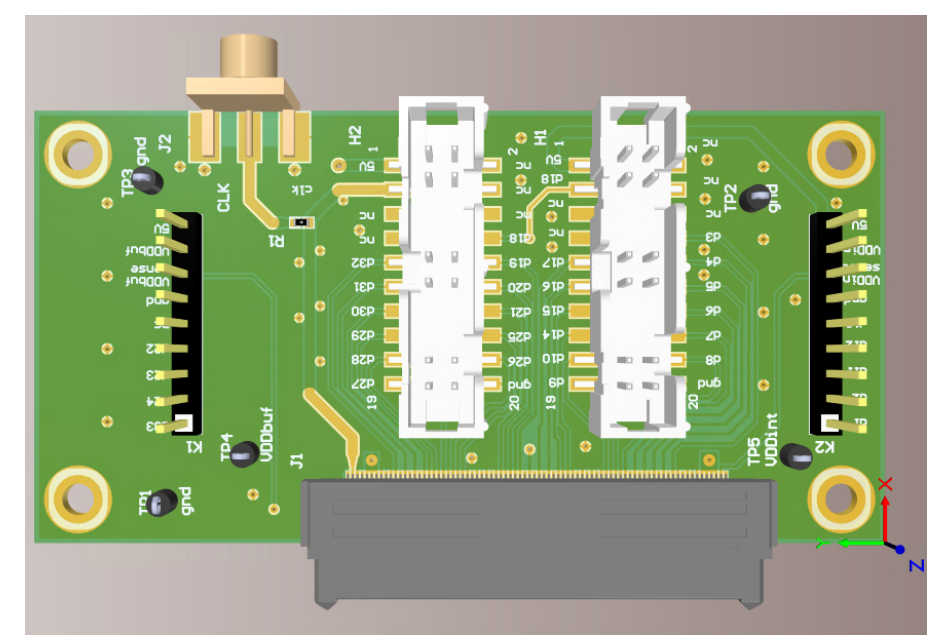

Figure A.9: Probe card v1 (2x20-pin 0.1" connectors) top side view (image from Altium Designer). 
An alternative clock path through board SMA was added to the probe card for redundancy, termination, and monitoring purposes.

The output of TDC is routed from the board-to-board connector to the pod connectors using the top layer to avoid using via which adds impedance discontinuity. The routing was done using a ground-signal-ground configuration (GSG), similar to the core card design (Section A.1.3.

Numerous test points were added to the board to enable easy probing and debug during measurements. All unused PCB areas of the top and bottom copper layers were assigned to the board ground net and then uniformly stitched across the board through vias. Mounting holes were also added to the board for mechanical stability and robust board-to-board connection.

\section{A.2.2 Probe Card V2}

This variant of the probe card is meant to provide an interface option with the much newer and overall better Agilent 16903B from that uses 16950 logic analyzer. This probe card is assumed to be the primary test vehicle for characterization of LPI-TDC.

The main risk of this solution is the assembly challenge, since the connector used to interface with the logic analyzer has significantly tighter mechanical tolerances compared to probe card v1. The more sophisticated logic analyzer allows for higher degree of flexibility in trigger (sampling) clock options, including fully differential signalling and external voltage references. These options were designed into the board for safety and are part of the auxilary circuitry in schematic terms. The output bus routing, power domains, ground, and mechanical handling was done similar to probe card v1 [see chapter prove card v1]. 


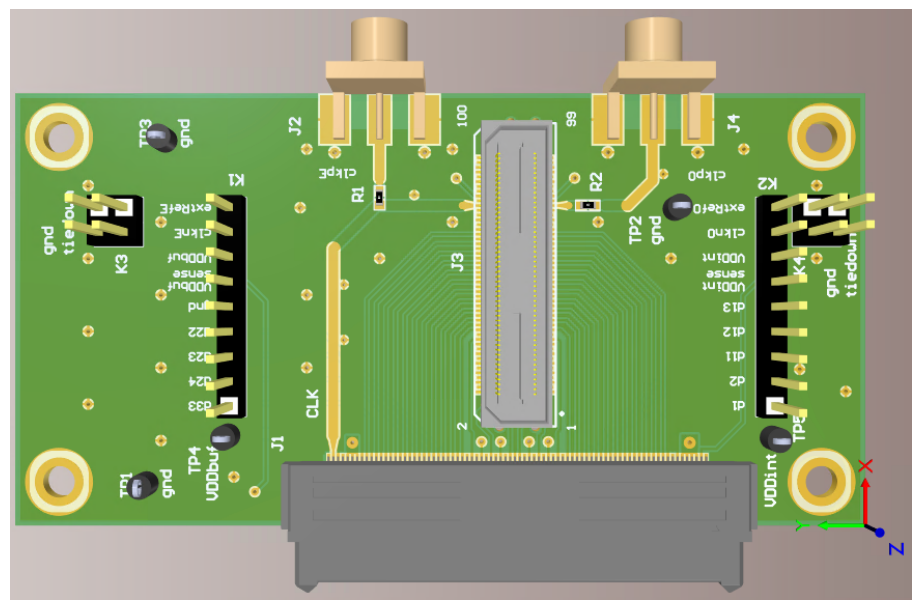

Figure A.10: Probe card v2 (high density connector) top side view (image from Altium Designer).

\section{A.3 Board Assembly and Debug}

Due to fine pitch of various components and surface-mounted soldering requirements, a heat re-flow oven, retrofitted from an off-the-shelf toaster oven was used (Figure A.11). A solder re-flow profile was created and experimentally calibrated for the thermal inertia of this particular oven.

A total of 5 core cards were assembled and one of each probe cards (v1 and v2). For ease of component assembly and placement, a stencil for solder paste application was ordered from the board fabrication facility (PCBway) at the time of design submission. To further simplify component placement and avoid errors, assembly drawings were generated to streamline the placement of all surface-mount passive components such as capacitors and resistors.

The bring up process was done methodically using the following steps:

1. Assembled the core card v1

2. performed DC probing before placing any components

3. Place and solder all board components except the DUT (ICGCUKIK) 


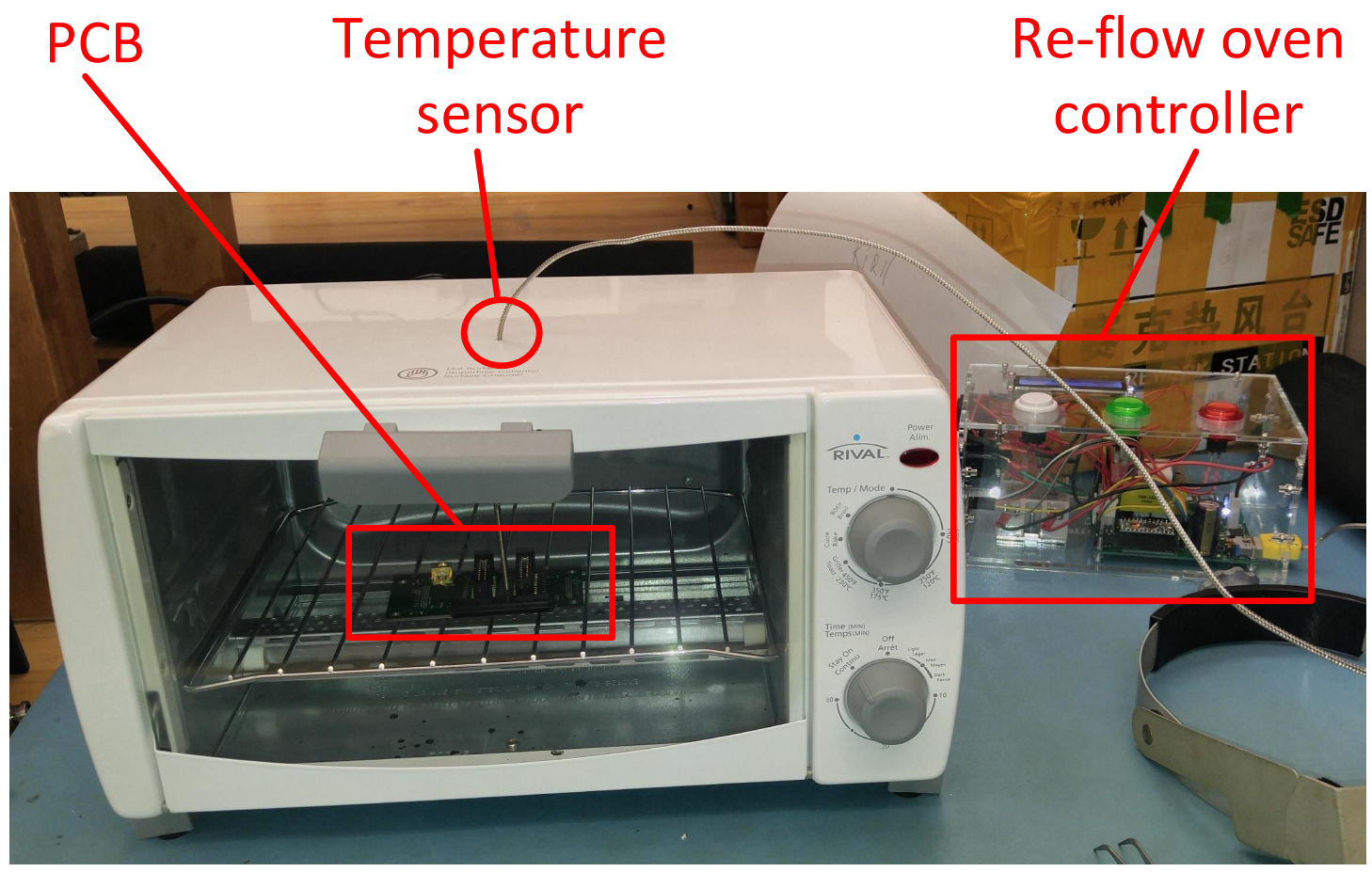

Figure A.11: Re-flow oven setup used to assemble the PCB with delicate surfacemount components.

re-test DC connectivity for short/open

rework any component as required

4. Populate the DUT on core card v1

5. perform final DC checks on core card

6. repeat above steps (with exception of the DUT) for probe card v1 and v2

\section{A.4 Summary of PCB Design}

Using the above methodology yielded a fully-functional modular system, with the exception of 2 silk screen errors (board component labels). The most challenging aspect of assembly was installing the fine pitch 100-pin connector for probe card v2 
logic analyzer. The second most challenging item was the board-to-board connector assembly. In both cases the issue was mainly due to either too much or too little solder paste causing opens or shorts. With some practice, a correct amount of solder paste and re-flow recipe was determined, producing an operational test system. The availability of two probe card variants proved to be effective in early debugging the system connectivity checks. 


\section{Appendix B}

\section{Matlab Test Bench Environment}

Listing B.1: Experiment automation using MATLAB: main run control script.

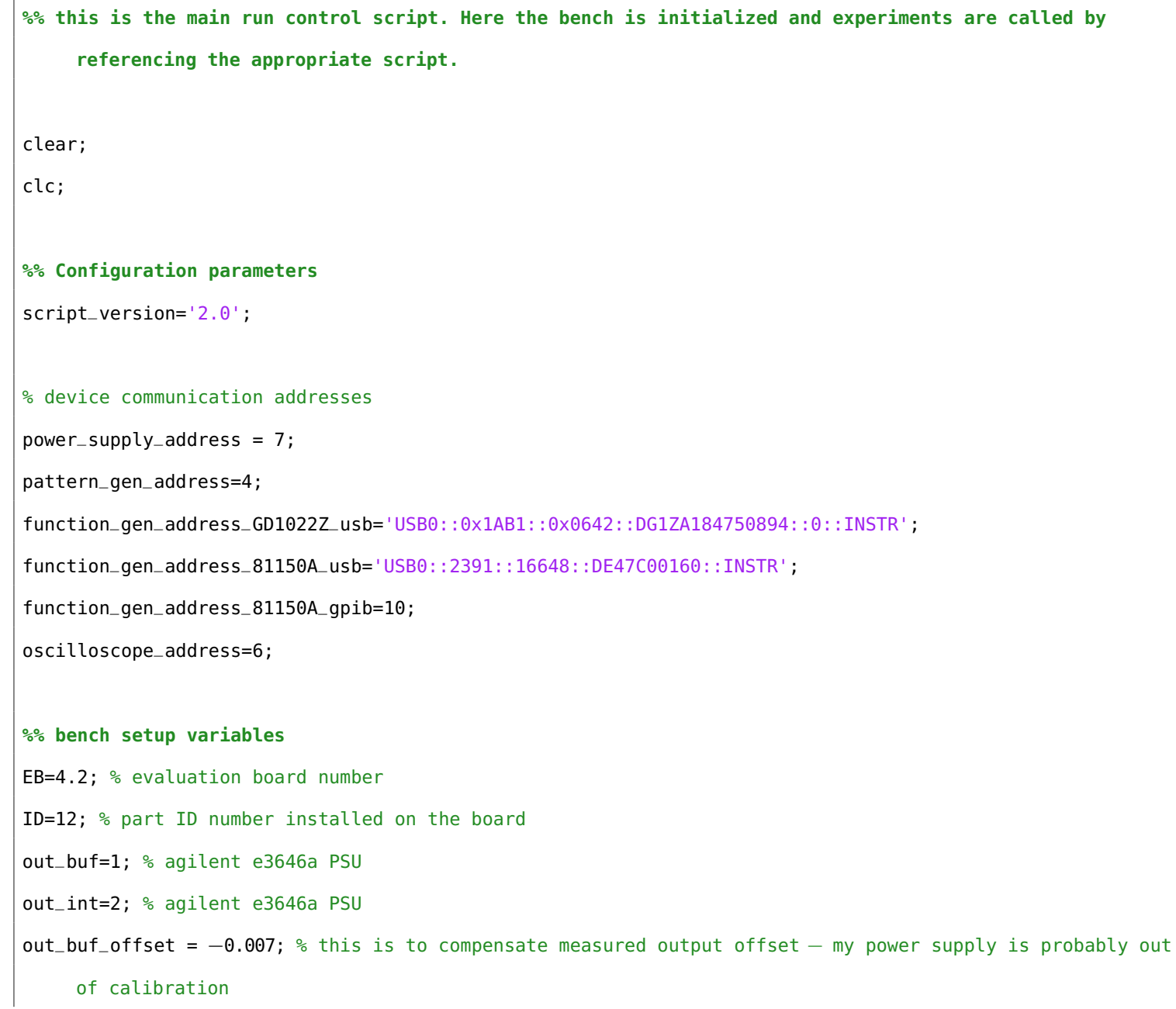




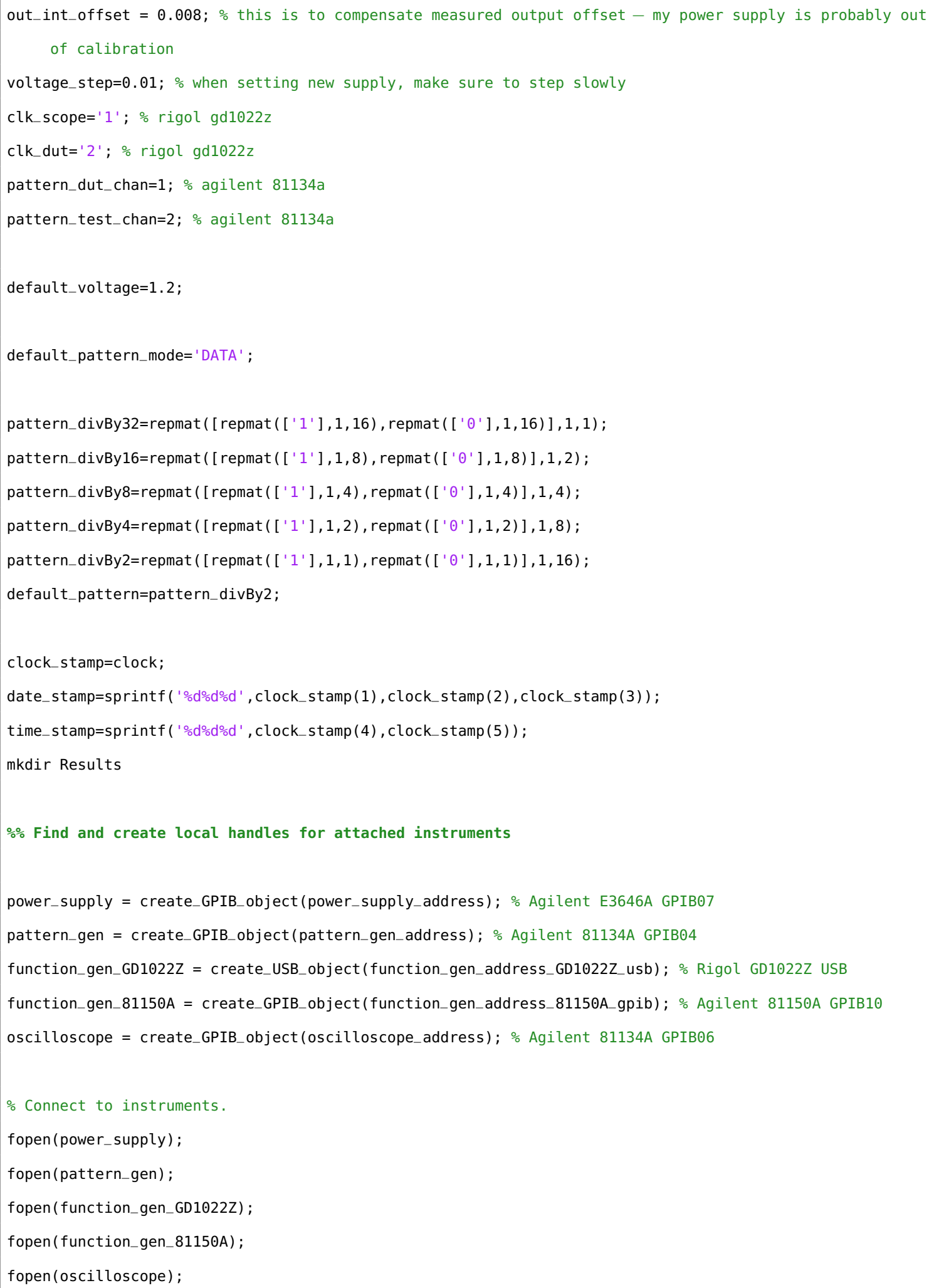


61

62

63

69

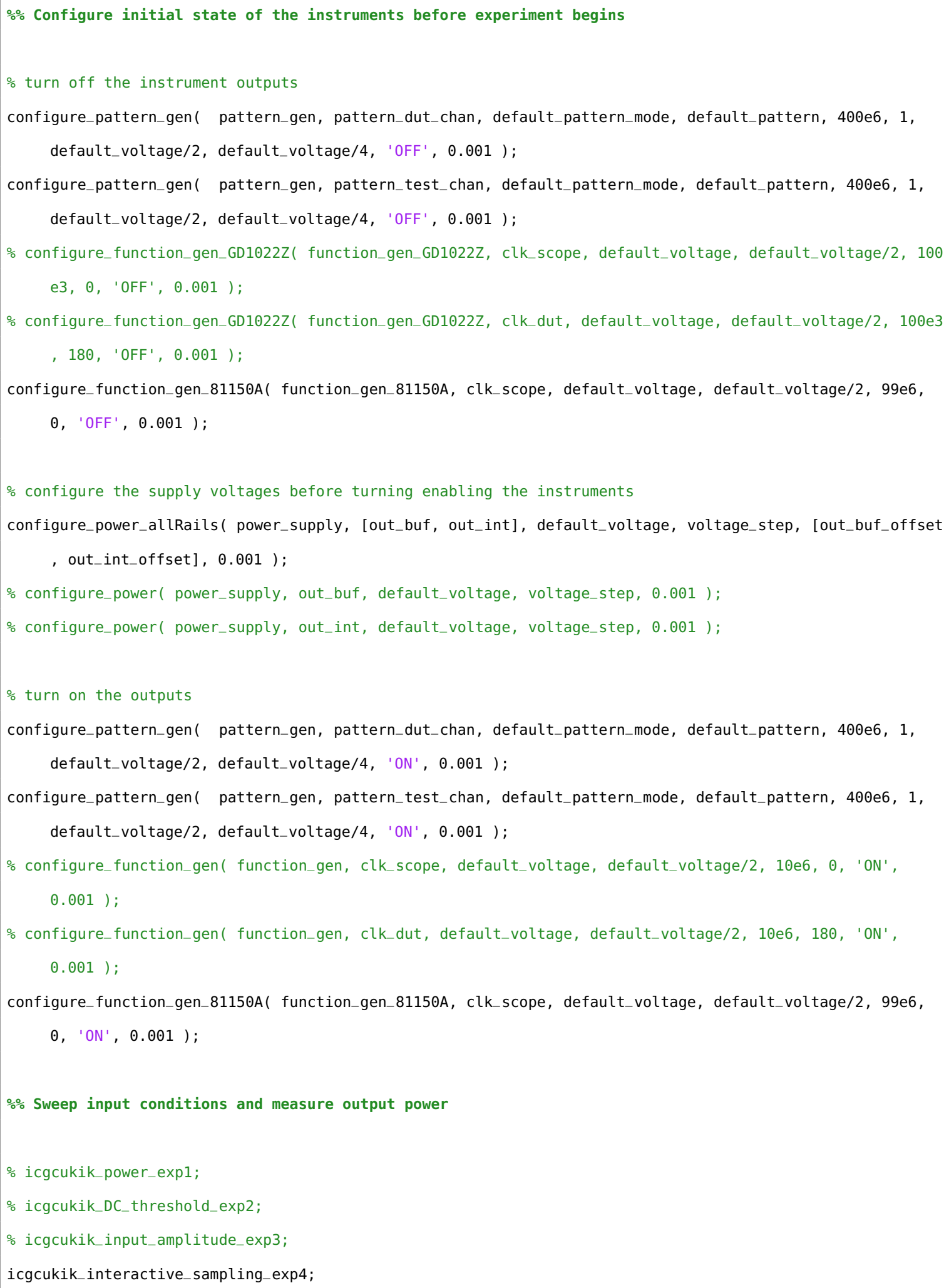


89

90

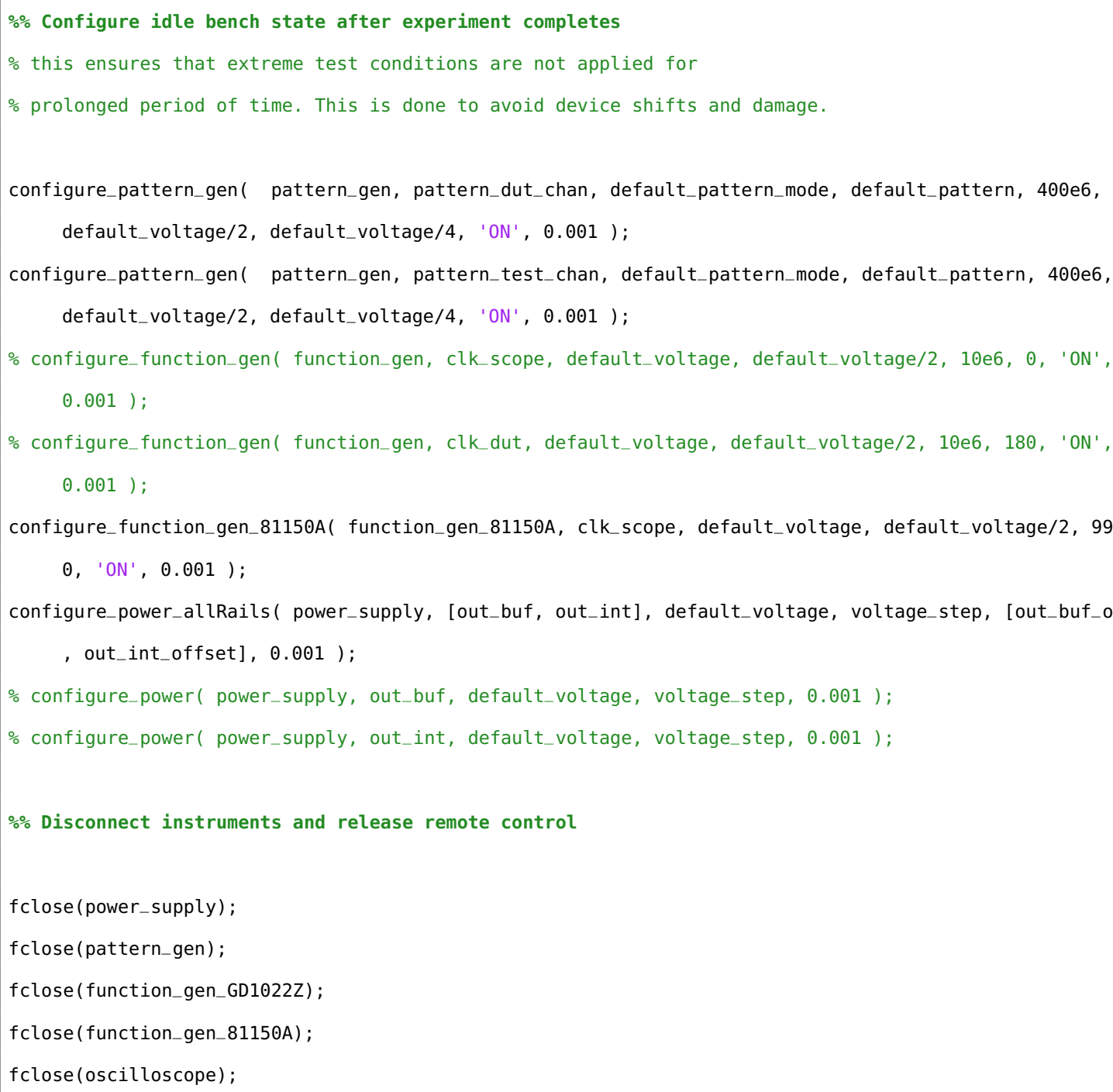


Listing B.2: Experiment automation using MATLAB: interactive experiment flow.

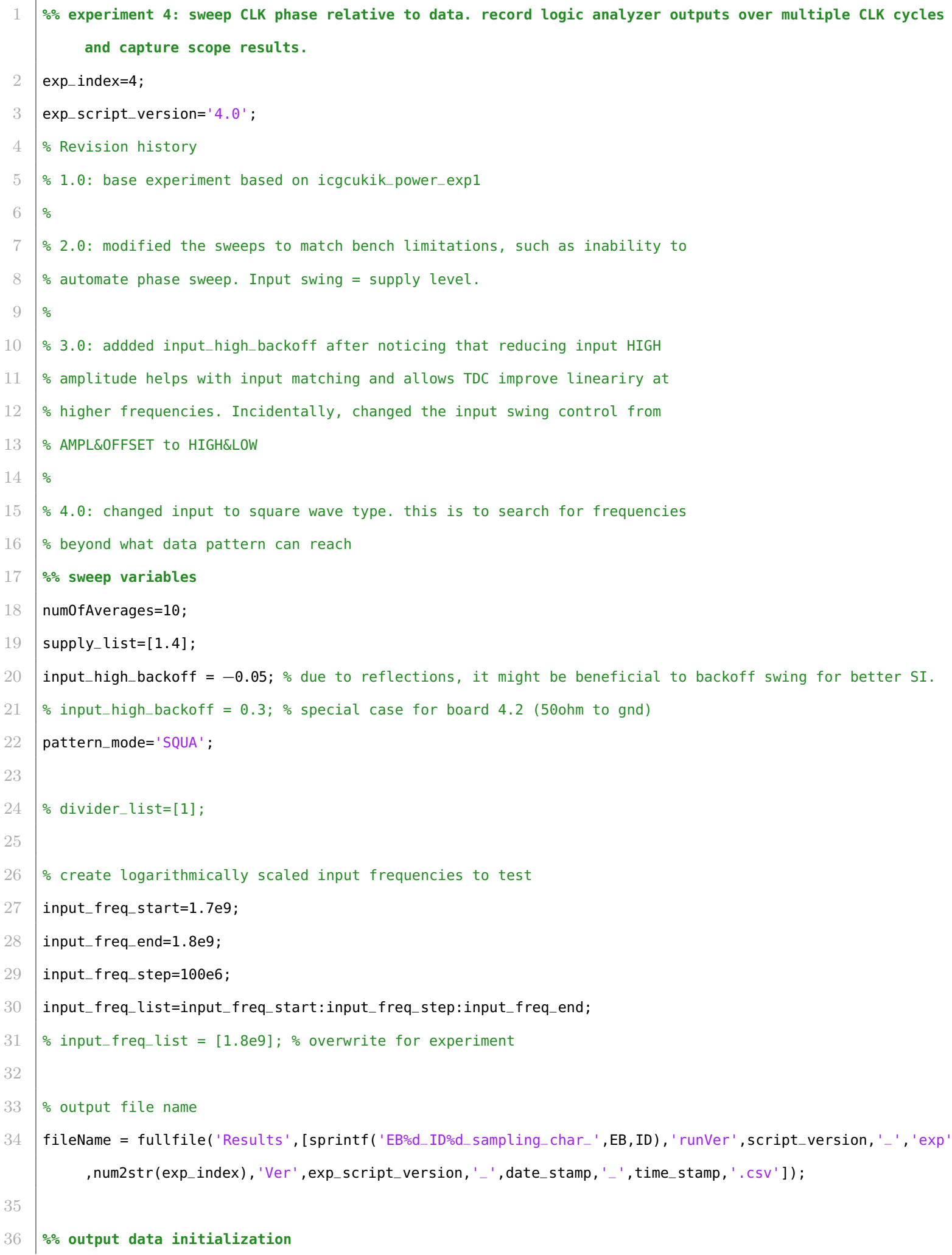




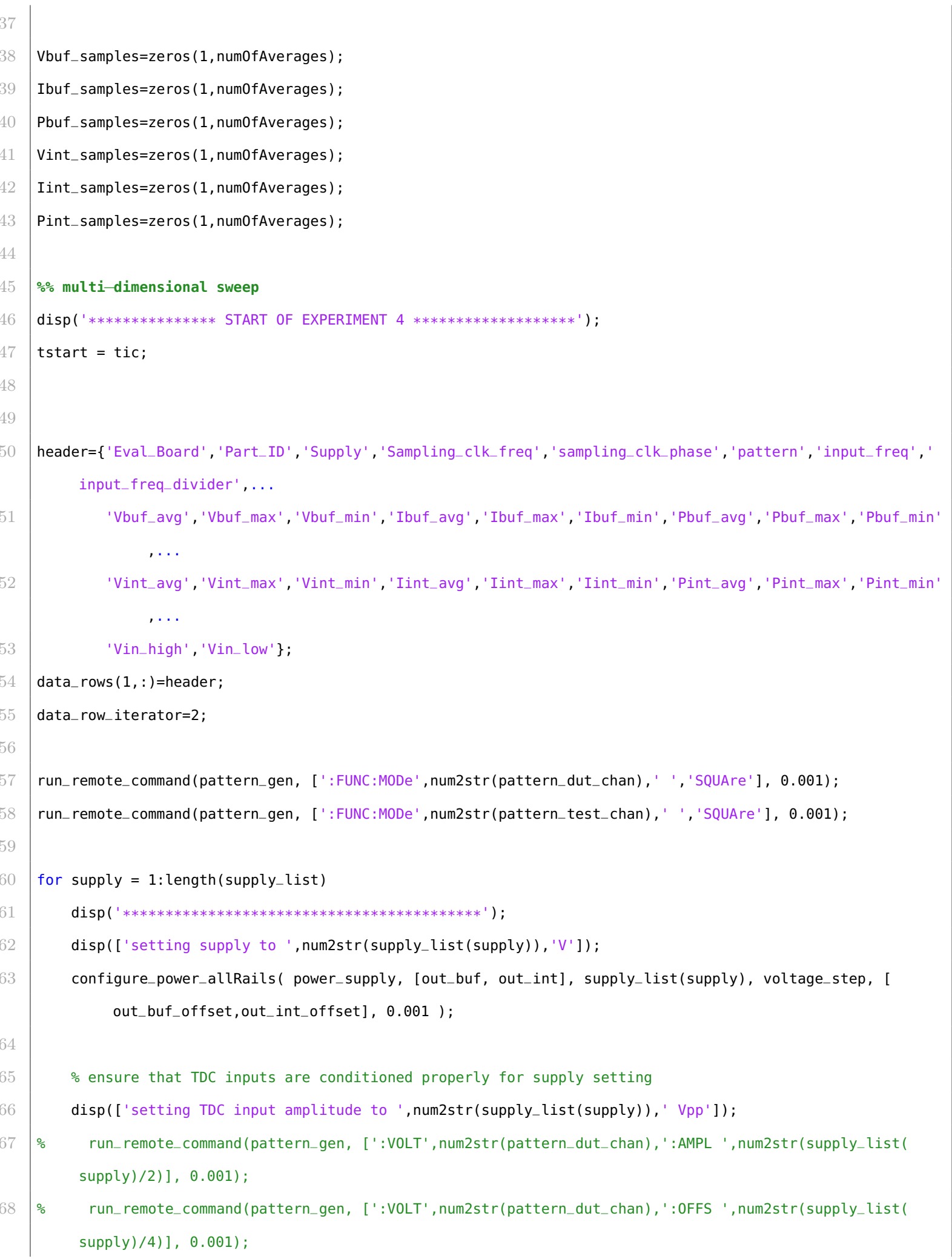


run_remote_command(pattern_gen, [ ' :VOLT', num2str(pattern_dut_chan), ':HIGH ' ,num2str( (supply_list( supply)+input_high_backoff)/2)], 0.001$)$; run_remote_command (pattern_gen, [ ':VOLT', num2str(pattern_dut_chan), ':LOW ',num2str (0) ], 0.001$)$; run_remote_command(pattern_gen, [ ' : VOLT' , num2str(pattern_test_chan), ' :HIGH ' ,num2str( (supply_list( supply)+input_high_backoff)/2)], 0.001); run_remote_command(pattern_gen, [ ':VOLT', num2str(pattern_test_chan), ': LOW ', num2str $(0)$ ], 0.001$)$; run_remote_command(pattern_gen, [ ' :VOLT', num2str(pattern_dut_chan), ' :HIGH ', num2str( (supply_list( supp(y)+input_high_backoff))], 0.001); 


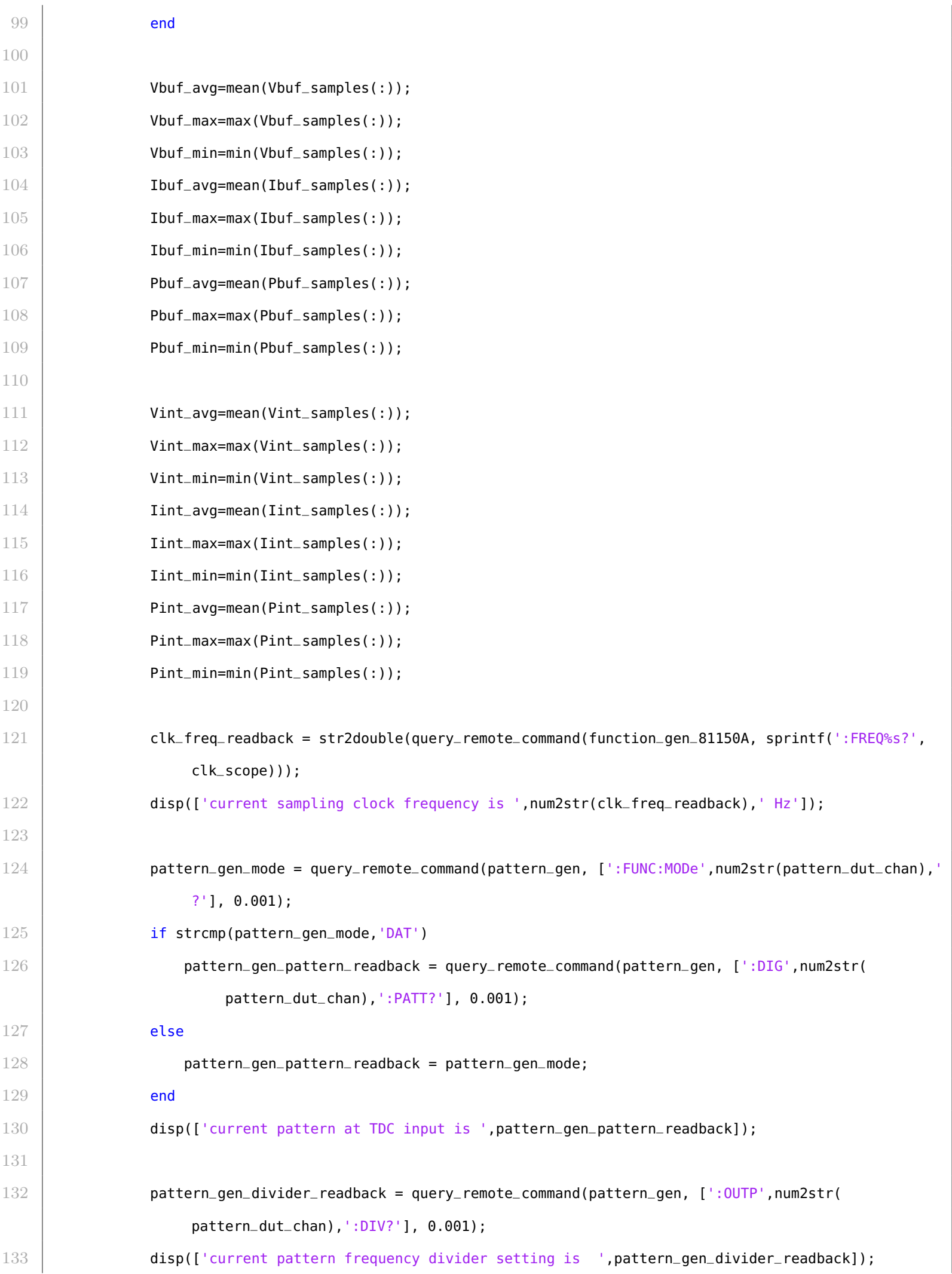




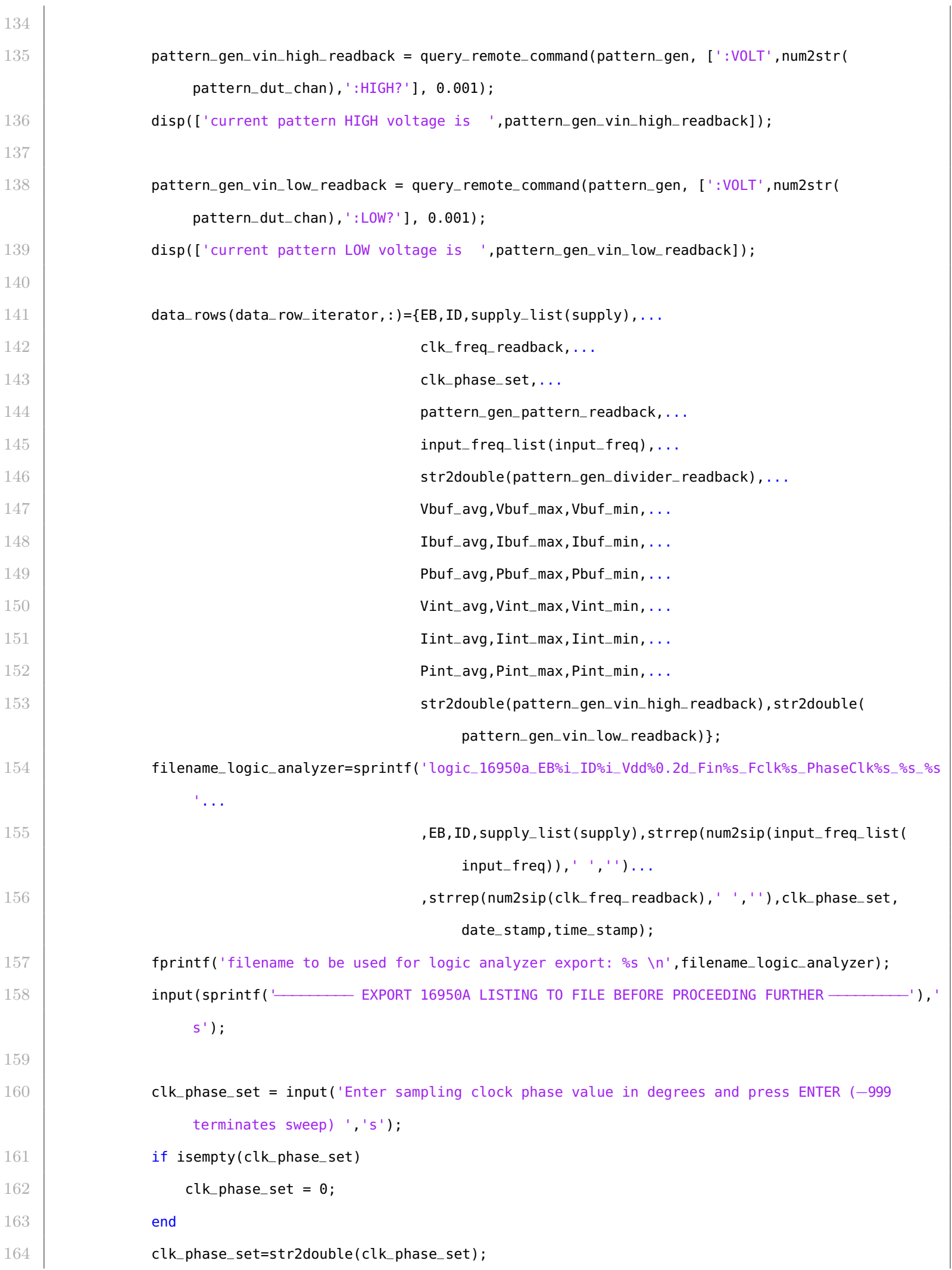




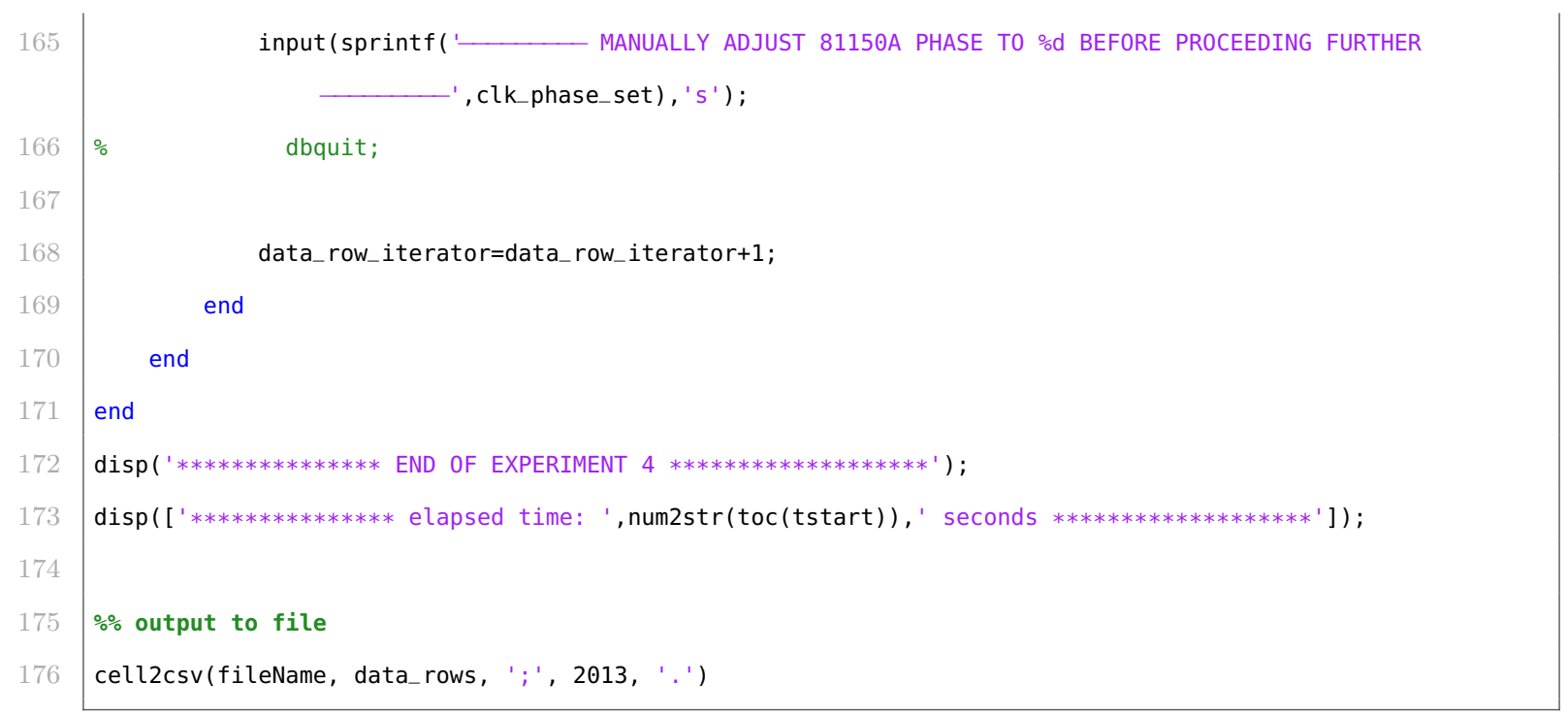

